\title{
The Dynamics, Structure, and Fate of a Young Cluster during Gas Dispersal: Hectoschelle, Chandra, Spitzer, and Gaia Observations of Cep OB3b
}

\author{
N. Karnath ${ }^{1}$, J. J. Prchlik ${ }^{2}$ (D) R. A. Gutermuth ${ }^{3}$ (D) T. S. Allen ${ }^{4,5}$, S. T. Megeath ${ }^{1}$ (D) J. L. Pipher ${ }^{6}$ (D), S. Wolk ${ }^{2}$ (D) and R. D. Jeffries ${ }^{7}$ \\ ${ }^{1}$ Department of Physics and Astronomy, University of Toledo, Toledo, OH 43606, USA; nicole.karnath@ rockets.utoledo.edu, s.megeath@utoledo.edu \\ ${ }^{2}$ Harvard-Smithsonian Center for Astrophysics, 60 Garden St., Cambridge, MA 02138, USA \\ ${ }^{3}$ Department of Astronomy, University of Massachusetts, Amherst, MA 01003, USA \\ ${ }^{4}$ Portland State University 1825 SW Broadway Portland, OR 97207, USA \\ 5 Lowell Observatory, 1400 West Mars Hill Road, Flagstaff, AZ 86001, USA \\ ${ }^{6}$ Department of Physics and Astronomy, University of Rochester, Rochester, NY 14627, USA \\ 7 Astrophysics Group, Keele University, Keele, Staffordshire ST5 5BG, UK \\ Received 2018 September 29; revised 2018 November 6; accepted 2018 November 9; published 2019 January 21
}

\begin{abstract}
We present a study of the kinematics and structure of the Cep OB3b cluster based on new spectra obtained with the Hectoschelle spectrograph on the MMT and data from Spitzer, Chandra, and Gaia. At a distance of $819 \pm 16$ pc, Cep OB3b is one of the closest examples of a young ( $\sim 3-5 \mathrm{Myr})$, large ( 3000 total members) cluster at the late stages of gas dispersal. The cluster is broken into two subclusters surrounded by a lower density halo. We fit the empirical density law of King to each subcluster to constrain their sizes and structure. The richer eastern subcluster has circular symmetry, a modest central density, and lacks molecular gas toward its core, suggesting it has undergone expansion due to gas dispersal. In contrast, the western subcluster deviates from circular symmetry, has a smaller core size, and contains significant molecular gas near its core, suggesting that it is in an earlier phase of gas dispersal. We present posterior probability distributions for the velocity dispersions from the Hectoschelle spectra. The east will continue to expand and likely form a bound cluster with $\sim 35 \%$ of stars remaining. The west is undergoing slower gas dispersal and will potentially form a bound cluster with $\sim 75 \%$ of stars remaining. If the halo dissipates, this will leave two independent clusters with $\sim 300$ members; proper motions suggest that the two subcluster are not bound to each other.
\end{abstract}

Key words: open clusters and associations: Cep OB3b - stars: formation - stars: kinematics and dynamics - stars: pre-main sequence - techniques: radial velocities

\section{Introduction}

Most stars form in embedded clusters (Carpenter 2000; Lada \& Lada 2003; Megeath et al. 2016), and within a few Myr, the natal gas will be expelled from the cluster, relieving it of internal extinction assuming dust moves with the gas. Given that star formation efficiency (SFE) is low, $4 \%$ for entire clouds and 20\% in clusters (Lada \& Lada 2003; Allen et al. 2007; Evans et al. 2009; Gutermuth et al. 2011b; Megeath et al. 2016), most of the mass leaves the cluster in a few Myr, drastically lowering the gravitational potential of the cluster. The ensuing dynamical evolution will determine whether the cluster - in whole or in part—will form a bound open cluster or disperse into the galactic disk. Cep OB3b is an excellent environment to study this crucial step of evolution because at 3-5 Myr (Littlefair et al. 2010; Allen et al. 2012), the cluster has dispersed most of its natal gas and is observable at visible wavelengths. This gives a snapshot of cluster evolution toward the end of gas dispersal, in a birth cluster similar to that of the Sun (Adams et al. 2001), and at a later stage of evolution than the Orion Nebula Cluster (ONC).

Many numerical studies have been carried out to determine how clusters evolve during and after gas dispersal using a range of assumptions for the initial cluster properties and the timescale for gas dispersal (e.g., Lada et al. 1984; Adams 2000; Geyer \& Burkert 2001; Boily \& Kroupa 2003; Baumgardt \& Kroupa 2007; Chen \& Ko 2009; Goodwin 2009; Proszkow et al. 2009; Moeckel \& Bate 2010; Pelupessy \& Portegies Zwart 2012; Farias et al. 2015, 2018). Observational studies are limited at this crucial time during gas dispersal. Most of the large, young clusters within $1 \mathrm{kpc}$, in particular Orion, NGC 2264 , or Mon R2, are partially embedded and appear to be in earlier stages of their gas dispersal (Dahm \& Simon 2005; Gutermuth et al. 2011b; Rapson et al. 2014). Comparisons of the number of embedded and bound clusters within $\sim 2 \mathrm{kpc}$ of the Sun indicate $7 \%$ of embedded clusters survive gas expulsion to form open clusters (Lada \& Lada 2003). Determining the structure and kinematics of clusters undergoing gas dispersal is a key step toward improving our understanding of what factors determine whether a cluster survives.

Allen et al. (2012) carried out a census of the young stellar objects (YSOs) in Cep OB3b. They employed Spitzer data to identify stars with infrared excesses due to dusty disks or envelopes, a combination of new and archival Chandra data to detect X-ray emission from coronae of young stars, and visible light photometry from the literature to identify stars on the Cep OB3b isochrone. Allen et al. (2012) estimated that there are $\sim 3000$ total members in Cep OB3b consisting of primarily low-mass $\left(\leqslant 1 M_{\odot}\right)$ stars. The density of stars is considerably less than the ONC (Hillenbrand \& Hartmann 1998; Megeath et al. 2016), but it is comparable in size and membership to the ONC (Carpenter 2000; Allen et al. 2007), making Cep OB3b one of the largest known, young clusters within $1 \mathrm{kpc}$ of the Sun (Allen et al. 2012). Most of the members, however, lie in a cavity of low extinction, implying that most of the natal gas has been dispersed. This combination of size and evolutionary state makes it an excellent region to study the effect of gas dispersal on young clusters. The spatial distribution of the objects shows 
Table 1

Hectoschelle Observations

\begin{tabular}{lccccc}
\hline \hline UT Date & $\begin{array}{c}\text { R.A. } \\
(\mathrm{J} 2000)\end{array}$ & $\begin{array}{c}\text { Decl. } \\
(\mathrm{J} 2000)\end{array}$ & $\begin{array}{c}\text { Exposures } \\
(\# \times \mathrm{s})\end{array}$ & $\begin{array}{c}\text { Seeing } \\
(\operatorname{arcsec})\end{array}$ & Airmass \\
\hline 2009 Oct 26 & 225512.36 & +624011.64 & $4 \times 1800$ & 1.97 & 1.26 \\
2009 Nov 03 & 225509.12 & +623907.56 & $4 \times 1800$ & 1.76 & 1.23 \\
2010 Nov 25 & 225507.86 & +624216.02 & $4 \times 1800$ & 1.83 & 1.23 \\
2011 Oct 20 & 225508.04 & +624135.52 & $4 \times 1800$ & 0.72 & 1.25 \\
2013 June 25 & 225543.68 & +623903.24 & $4 \times 2100$ & 1.74 & 1.53 \\
\hline
\end{tabular}

a hierarchical morphology composed of two distinct subclusters, denoted east (eastern subcluster) and west (western subcluster), each associated with a distinct molecular clump within the larger Cep OB3 molecular cloud (Sargent 1977; Heyer et al. 1996). Furthermore, a substantial difference in the disk fraction of the subclusters, $32 \% \pm 4 \%$ for the east and $50 \% \pm 6 \%$ for the west, was found by Allen et al. (2012) and was attributed to a difference in the typical ages of the constituent stars (east being older) in the subclusters, rather than the photoevaporation of disks by high-mass members. These results suggest a distinct origin for both subclusters; however, since the two subclusters share a contiguous region of high stellar surface density (Gutermuth et al. 2011a; Allen et al. 2012) within the Cep OB3b cloud with a diffuse halo of stars surrounding it, we consider these as parts of a single cluster. Cep OB3b has one O star (O7V, HD 217086) that resides in the eastern subcluster and several B stars throughout the entire cluster (Blaauw et al. 1959).

In order to relate young cluster populations to older stars in the field and open clusters, we need to observe young clusters at this critical stage of their evolution. The goal of this paper is to assess the kinetic and potential energy of the two subclusters, determine their fates as bound clusters, and study the effect of gas dispersal on young clusters. We carry out a radial velocity (RV) survey of 499 stars in Cep OB3b to measure the velocity dispersion and measure the kinetic energy. This study is complemented by an analysis of the structure of the two subclusters using Spitzer and Chandra data; from these data we can determine the current potential energy of the cluster. Prior to this study, the structure and kinematics of Cep OB3b have not been assessed using the population of low-mass stars. Finally, we use Gaia DR2 to both refine the distance to the cluster, which is needed to measure the potential energy of the cluster, and to measure the bulk motions of the subclusters.

Velocity dispersions of YSOs have been measured for a number of young clusters and molecular clouds probing their kinematical states. The ONC has been observed several times in $\mathrm{RV}$ surveys to determine velocity dispersions: $\sim 1.8 \mathrm{~km} \mathrm{~s}^{-1}$ (Sicilia-Aguilar et al. 2005), $\sim 2.3 \mathrm{~km} \mathrm{~s}^{-1}$ (Fúrész et al. 2008), $\sim 2.5 \mathrm{~km} \mathrm{~s}^{-1}$ (Tobin et al. 2009), $\sim 2.3 \mathrm{~km} \mathrm{~s}^{-1}$ (Kounkel et al. 2016), and $\sim 1.7 \mathrm{~km} \mathrm{~s}^{-1}$ (Da Rio et al. 2017). Da Rio et al. (2014) found that the ONC is an expanding cluster undergoing gas expulsion, which is in agreement with Kuhn et al. (2018), who measured a parallax-based velocity dispersion from Gaia DR2 data of $\sim 1.8 \mathrm{~km} \mathrm{~s}^{-1}$.

Outside of Orion, Hectoschelle observations of NGC 2264 by Fürész et al. (2006) and Tobin et al. (2015) found a onedimensional velocity dispersion of $\sim 2.5 \mathrm{~km} \mathrm{~s}^{-1}$ and classified the region as several clumps of star formation and not as one bound cluster. NGC 1333 was studied with APOGEE, which measured a velocity dispersion of $\sim 1 \mathrm{~km} \mathrm{~s}^{-1}$, yielding a virialized cluster with some initial substructure (Foster et al. 2015). The APOGEE project also observed IC 348, measuring a velocity dispersion of $\sim 0.7 \mathrm{~km} \mathrm{~s}^{-1}$ and implying a supervirial state; consistent with IC 348 being in an advanced phase of gas dispersal (Cottaar et al. 2015). Ortiz-León et al. (2018) used the GAIA DR2 release on IC 348 and NGC 1333 and found velocity dispersions that agree with the APOGEE project.

The Gaia-ESO survey studied several young regions, probing their kinematical structure: Jeffries et al. (2014) determined there were two kinematical populations in $\gamma \mathrm{Vel} \mathrm{B}$ with velocity dispersions of 1.6 and $0.34 \mathrm{~km} \mathrm{~s}^{-1}$, Rigliaco et al. (2016) determined a velocity dispersion of $1.14 \mathrm{~km} \mathrm{~s}^{-1}$ in L1688 in the $\rho$ Ophiuchi molecular cloud, and Sacco et al. (2017) recorded a velocity dispersion of $1.1 \mathrm{~km} \mathrm{~s}^{-1}$ in Chamaeleon I. For post-gas-dispersal clusters, Jeffries et al. (2006) determined there are two spatially superimposed components in $\sigma$ Ori with velocity dispersions of 1.1 and $1.3 \mathrm{~km} \mathrm{~s}^{-1}$, and Kuhn et al. (2014) studied NGC 6231 in the Sco OB1 association and found it is gravitationally bound.

In Section 2, we describe our observations and data reduction. In Section 3, we provide our analysis, and Section 4 contains the results. We discuss implications of the results in Section 5 and summarize the paper in Section 6.

\section{Hectoschelle Observations and Data Reduction}

The spectra were obtained on the MMT with Hectoschelle (Szentgyorgyi et al. 1998), a fiber-fed, echelle spectrograph with a resolving power of $\sim 32,000$. The 240 fibers are robotically placed on the maximum possible number of target stars for a given configuration. Five epochs of observations were taken over five years (2009-2013; see Table 1) using the 25th order; these spectra span $150 \AA$ centered on $\mathrm{H} \alpha$ at $6563 \AA$ A. Every configuration had $\sim 30$ fibers placed on the sky to measure the sky emission, and the remaining fibers were placed on target stars with integration times of $4 \times 1800 \mathrm{~s}$, except for the final epoch, which was $4 \times 2100 \mathrm{~s}$. In addition, one set of spectra was obtained with the telescope offset by $5^{\prime \prime}$ from the target stars per configuration to measure the contribution of interstellar lines in the spectra.

The target stars are from the combined visible, infrared, and X-ray study of Allen et al. (2012). They determined membership by the detection of an IR excess with Spitzer IR using color-color diagrams, by the detection of X-ray emission from enhanced coronal activity using novel and archival Chandra data, and-less reliably-by their coincidence in $V$ versus $V-I$ diagrams with the isochrone of members identified with the X-ray and Spitzer data. Diskless members with X-ray detections were the highest priority targets of the survey since $\mathrm{H} \alpha$ emission dominates the spectrum of disk objects due to accretion. Spitzer-identified YSOs with disks, both Class II and 
Table 2

2009.1026 RV Results

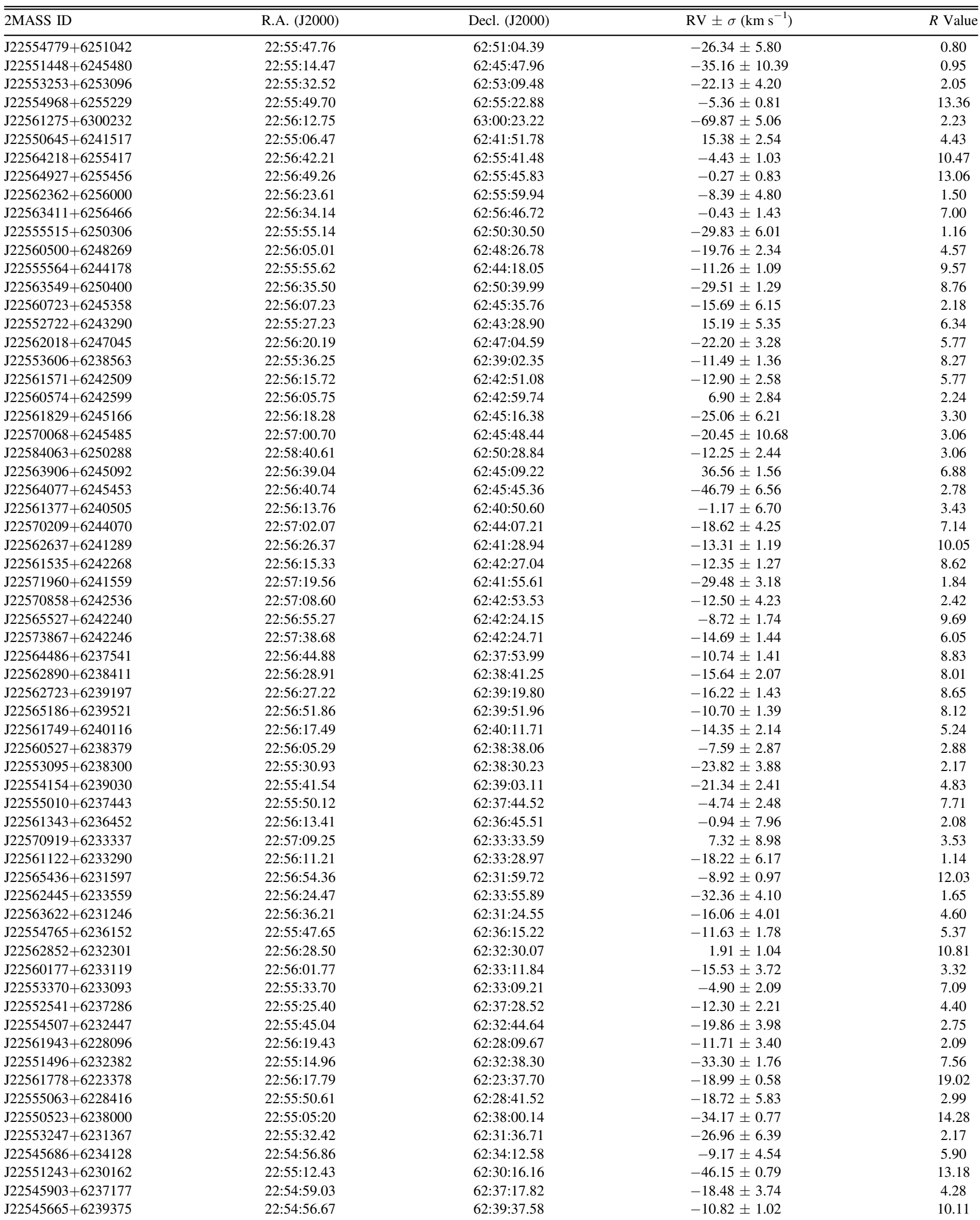


Table 2

(Continued)

\begin{tabular}{|c|c|c|c|c|}
\hline 2MASS ID & R.A. (J2000) & Decl. (J2000) & $\mathrm{RV} \pm \sigma\left(\mathrm{km} \mathrm{s}^{-1}\right)$ & $R$ Value \\
\hline $\mathrm{J} 22545364+6234031$ & $22: 54: 53.61$ & $62: 34: 03.10$ & $-18.92 \pm 3.57$ & 5.32 \\
\hline $\mathrm{J} 22543747+6225456$ & $22: 54: 37.47$ & $62: 25: 45.69$ & $-31.61 \pm 7.49$ & 0.84 \\
\hline $\mathrm{J} 22543882+6223364$ & $22: 54: 38.82$ & $62: 23: 36.61$ & $-11.39 \pm 0.79$ & 13.85 \\
\hline $\mathrm{J} 22545101+6239064$ & 22:54:50.99 & $62: 39: 06.53$ & $-22.20 \pm 8.00$ & 1.32 \\
\hline $\mathrm{J} 22533383+6223183$ & $22: 53: 33.82$ & $62: 23: 18.13$ & $7.09 \pm 0.88$ & 12.23 \\
\hline $\mathrm{J} 22541607+6230398$ & $22: 54: 16.07$ & $62: 30: 39.76$ & $-19.90 \pm 1.10$ & 9.55 \\
\hline $\mathrm{J} 22535527+6233218$ & $22: 53: 55.26$ & $62: 33: 21.76$ & $-21.93 \pm 6.63$ & 3.07 \\
\hline $\mathrm{J} 22542077+6236223$ & $22: 54: 20.75$ & $62: 36: 22.42$ & $51.74 \pm 1.61$ & 7.51 \\
\hline $\mathrm{J} 22542405+6235158$ & $22: 54: 24.04$ & $62: 35: 15.95$ & $-1.88 \pm 4.77$ & 5.65 \\
\hline $\mathrm{J} 22530748+6225036$ & 22:53:08.57 & $62: 25: 01.54$ & $-21.95 \pm 7.81$ & 1.59 \\
\hline $\mathrm{J} 22544752+6240001$ & $22: 54: 47.51$ & $62: 40: 00.16$ & $-25.52 \pm 1.33$ & 7.80 \\
\hline $\mathrm{J} 22523087+6231116$ & $22: 52: 30.84$ & $62: 31: 11.45$ & $-13.52 \pm 6.06$ & 1.67 \\
\hline $\mathrm{J} 22541116+6235541$ & $22: 54: 11.14$ & $62: 35: 54.21$ & $-10.40 \pm 1.62$ & 6.04 \\
\hline $\mathrm{J} 22534360+6235322$ & $22: 53: 43.60$ & $62: 35: 32.20$ & $-38.99 \pm 6.97$ & 2.37 \\
\hline $\mathrm{J} 22540438+6235548$ & $22: 54: 04.37$ & $62: 35: 54.68$ & $-11.81 \pm 5.33$ & 4.45 \\
\hline $\mathrm{J} 22532966+6234051$ & $22: 53: 29.64$ & $62: 34: 05.00$ & $-21.55 \pm 2.02$ & 7.15 \\
\hline $\mathrm{J} 22540642+6236503$ & 22:54:06.40 & $62: 36: 50.37$ & $-4.72 \pm 0.97$ & 11.11 \\
\hline $\mathrm{J} 22532283+6237409$ & $22: 53: 22.85$ & $62: 37: 40.99$ & $-6.43 \pm 3.43$ & 3.67 \\
\hline $\mathrm{J} 22530628+6236326$ & 22:53:06.29 & $62: 36: 32.68$ & $-28.86 \pm 5.59$ & 1.17 \\
\hline $\mathrm{J} 22535723+6236506$ & $22: 53: 57.24$ & $62: 36: 50.70$ & $-58.37 \pm 2.02$ & 6.13 \\
\hline $\mathrm{J} 22531785+6234592$ & $22: 53: 17.86$ & $62: 34: 59.10$ & $-21.64 \pm 6.49$ & 3.00 \\
\hline $\mathrm{J} 22533489+6237007$ & $22: 53: 34.91$ & $62: 37: 00.69$ & $-7.12 \pm 2.14$ & 4.89 \\
\hline $\mathrm{J} 22540731+6238098$ & $22: 54: 06.84$ & $62: 38: 06.56$ & $-54.22 \pm 3.43$ & 8.78 \\
\hline $\mathrm{J} 22524589+6234125$ & $22: 52: 45.92$ & $62: 34: 12.53$ & $-18.73 \pm 4.46$ & 2.39 \\
\hline $\mathrm{J} 22533413+6238199$ & $22: 53: 33.19$ & $62: 38: 20.55$ & $-28.19 \pm 4.50$ & 3.10 \\
\hline $\mathrm{J} 22534448+6239061$ & $22: 53: 44.43$ & $62: 39: 06.52$ & $-49.20 \pm 3.43$ & 9.93 \\
\hline $\mathrm{J} 22541525+6239469$ & $22: 54: 15.24$ & $62: 39: 46.89$ & $-3.27 \pm 6.10$ & 0.71 \\
\hline $\mathrm{J} 22523061+6238279$ & $22: 52: 30.61$ & $62: 38: 28.15$ & $-18.59 \pm 1.05$ & 10.39 \\
\hline $\mathrm{J} 22530993+6239119$ & 22:53:09.91 & $62: 39: 12.16$ & $-0.36 \pm 1.26$ & 8.88 \\
\hline $\mathrm{J} 22541906+6241367$ & $22: 54: 19.05$ & $62: 41: 36.73$ & $-29.24 \pm 1.38$ & 9.13 \\
\hline $\mathrm{J} 22522918+6241092$ & $22: 52: 27.48$ & $62: 41: 10.30$ & $-38.69 \pm 4.28$ & 1.62 \\
\hline $\mathrm{J} 22525962+6243050$ & $22: 52: 59.63$ & 62:43:05.07 & $-25.75 \pm 0.64$ & 16.8 \\
\hline $\mathrm{J} 22541227+6241154$ & $22: 54: 12.29$ & $62: 41: 15.39$ & $-13.02 \pm 4.89$ & 1.08 \\
\hline $\mathrm{J} 22534690+6241504$ & $22: 53: 46.90$ & $62: 41: 50.54$ & $-92.68 \pm 5.74$ & 2.01 \\
\hline $\mathrm{J} 22522631+6242084$ & $22: 52: 26.32$ & $62: 42: 08.72$ & $-1.17 \pm 0.64$ & 16.89 \\
\hline $\mathrm{J} 22540632+6243500$ & $22: 54: 06.33$ & $62: 43: 50.09$ & $30.02 \pm 1.34$ & 7.61 \\
\hline $\mathrm{J} 22541162+6242285$ & $22: 54: 11.63$ & $62: 42: 28.55$ & $-28.24 \pm 10.86$ & 1.71 \\
\hline $\mathrm{J} 22543623+6241336$ & $22: 54: 36.23$ & $62: 41: 33.81$ & $-39.95 \pm 0.99$ & 10.77 \\
\hline $\mathrm{J} 22535500+6243412$ & $22: 53: 55.12$ & $62: 43: 36.89$ & $-20.80 \pm 5.16$ & 1.03 \\
\hline $\mathrm{J} 22535114+6245149$ & $22: 53: 51.12$ & $62: 45: 14.91$ & $-3.09 \pm 1.96$ & 5.12 \\
\hline $\mathrm{J} 22543280+6242318$ & $22: 54: 32.80$ & $62: 42: 31.86$ & $-17.64 \pm 7.89$ & 2.74 \\
\hline $\mathrm{J} 22542135+6248095$ & $22: 54: 21.35$ & $62: 48: 09.36$ & $-10.42 \pm 1.33$ & 8.11 \\
\hline $\mathrm{J} 22545080+6240485$ & $22: 54: 50.80$ & $62: 40: 48.55$ & $-11.79 \pm 1.20$ & 9.59 \\
\hline $\mathrm{J} 22542553+6244554$ & $22: 54: 25.55$ & $62: 44: 55.54$ & $-40.35 \pm 0.84$ & 12.68 \\
\hline $\mathrm{J} 22543071+6245323$ & $22: 54: 30.71$ & $62: 45: 32.30$ & $-74.41 \pm 3.91$ & 12.90 \\
\hline $\mathrm{J} 22540791+6248393$ & 22:54:07.86 & $62: 48: 39.48$ & $19.68 \pm 1.15$ & 9.78 \\
\hline $\mathrm{J} 22541238+6250443$ & $22: 54: 12.36$ & $62: 50: 44.34$ & $-11.25 \pm 3.81$ & 0.93 \\
\hline $\mathrm{J} 22542503+6249009$ & $22: 54: 25.05$ & $62: 49: 00.77$ & $8.21 \pm 0.92$ & 11.96 \\
\hline $\mathrm{J} 22541185+6253136$ & $22: 54: 11.88$ & $62: 53: 13.51$ & $0.22 \pm 4.40$ & 4.48 \\
\hline $\mathrm{J} 22550261+6251081$ & $22: 55: 01.39$ & $62: 51: 11.83$ & $-27.97 \pm 3.78$ & 5.39 \\
\hline $\mathrm{J} 22542910+6254472$ & $22: 54: 29.07$ & $62: 54: 47.07$ & $-24.82 \pm 5.42$ & 1.87 \\
\hline $\mathrm{J} 22545316+6250085$ & $22: 54: 53.16$ & $62: 50: 08.35$ & $-25.52 \pm 4.63$ & 2.34 \\
\hline $\mathrm{J} 22543257+6257160$ & $22: 54: 32.61$ & $62: 57: 15.97$ & $-35.62 \pm 8.34$ & 1.12 \\
\hline $\mathrm{J} 22552832+6254286$ & $22: 55: 30.02$ & $62: 54: 32.96$ & $12.58 \pm 1.28$ & 8.50 \\
\hline $\mathrm{J} 22552191+6251339$ & $22: 55: 21.17$ & $62: 51: 26.51$ & $-22.11 \pm 1.25$ & 8.21 \\
\hline $\mathrm{J} 22550699+6248278$ & $22: 55: 06.98$ & $62: 48: 27.96$ & $-4.91 \pm 1.11$ & 10.49 \\
\hline
\end{tabular}

transition-disk objects, were given second priority. Third priority was assigned to the potential members identified in the $V$ versus $V-I$ color-magnitude diagram; these would be diskless members without detectable $\mathrm{X}$-ray measurements. The lowest ranked objects were objects with a $V$-band magnitude of $\sim 11.5-14.5$ regardless of the potential for membership. These objects were targeted if no more fibers could be placed targeting any of the three higher ranked objects. A total of 561 


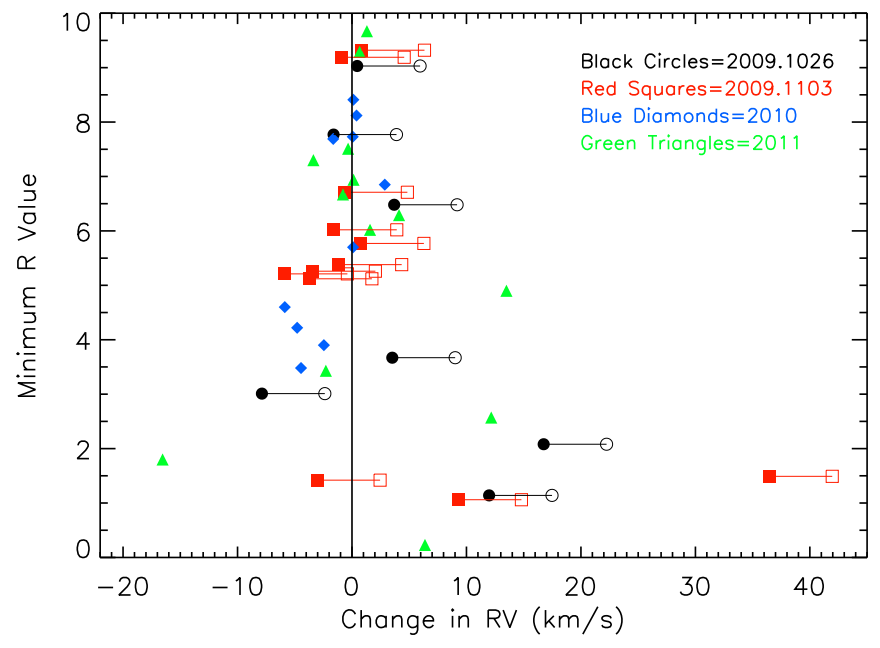

Figure 1. Minimum $R$ value vs. change in RV for objects observed in two epochs. The $x$-axis is the difference in RV between the two epochs. This excludes objects that were flagged as binaries in the initial cross-correlation. The observations from 2009.1026/1103 (black circles/red squares, respectively) have a systematic non-zero change in RV compared to the 2010 (blue diamonds) and 2011 (green triangles) epochs at high $R$. The RV results from both 2009 epochs were shifted by an offset of $5.5 \mathrm{~km} \mathrm{~s}^{-1}$; the open symbols show the change in the RV, and the filled symbols connected to these by a line show the change after the offset was applied.

spectra (over five epochs; see Tables 2-6) were taken toward Cep OB3b of 499 distinct stars, 190 of which were identified as members using the above criteria.

All spectra were reduced following the procedure in Szentgyorgyi (2006), which uses the standard IRAF reduction pipeline for echelle spectra. Cep OB3b has a significant amount of nebular emission stemming from the S155 HII region nearby. Spectra of the nebulosity taken at offset positions were used to subtract out the contribution from the nebular emission, as described in J.J. Prchlik et al. (2019, in preparation).

\section{Extraction of RVs}

We used the rvsao package (Kurtz \& Mink 1998) within IRAF to determine the RV of each spectrum. Synthetic spectral templates from Munari et al. (2005) were adopted for the crosscorrelation. The templates had $[\mathrm{Fe} / \mathrm{H}]=+0.5$ solar using the stellar atmosphere models from Kurucz (1993) that covered 2500-10500 A with a resolving power of 20,000. A supersolar metallicity was chosen to account for higher metallicity in younger stars compared to the Sun. Each spectrum was crosscorrelated with 11 templates ranging from 3500 to $6250 \mathrm{~K}$ in steps of $250 \mathrm{~K}$. We also cross-correlated with the higher resolution synthetic templates of Coelho et al. (2005). These provided solutions consistent with those using Munari et al., hence we adopted the RV values using the Munari et al. templates because they were closer in resolution to our data. The $\mathrm{H} \alpha$ region (6559-6566 $\AA$ ) was masked out of all spectra regardless of strength to avoid contamination of the extracted $\mathrm{RV}$. We did not account for potential broadening of the lines by stellar rotation. Regions in the spectra with high nebular contamination were also masked out individually by eye to avoid contamination of the measured RV.

The best matching template (highest $R$ value; Tonry \& Davis 1979) was selected, yielding the RV and uncertainty used in this analysis. The $R$ value corresponds to the quality of the cross-correlation and scales as $R=h / \sqrt{2} \sigma_{a}$, where $h$ is the height of the cross-correlation peak, and $\sigma_{a}$ is the estimated uncertainty from the rms of the antisymmetric portion of the correlation function. An RV range of -50 to $+50 \mathrm{~km} \mathrm{~s}^{-1}$ was used as an initial restriction. Each crosscorrelation plot was inspected by eye. Some objects had a strong peak outside of this range. For these objects, the range was extended and run again, and the highest $R$ value template result was adopted. Inspection by eye also enabled us to identify objects that were potential binaries. The RV values are given in Tables 2-7; all reported RVs are in terms of the local standard of rest, $V_{\text {lsr }}$.

The fifth epoch, 2013, observed 64 objects for a second time in addition to 13 objects not previously observed. This was useful to find binaries in the sample in addition to testing the consistency of the velocity calibration.

Figure 1 shows the difference in RV versus the minimum $R$ value of 42 objects that were observed twice and were not initially suspected as binaries based on the shape of their crosscorrelation plots (see Section 3.1). For 19 stars, we find a consistent offset between the 2009 epoch RVs compared to the 2013 measurement. This offset is not present when comparing the 2010 and $2011 \mathrm{RVs}$ to the $2013 \mathrm{RVs}$ for the 23 stars observed in those epochs.

In order to quantify the offset, we calculated a weighted average of the RV change between epochs with $R$ of 6 or higher, yielding $5.5 \mathrm{~km} \mathrm{~s}^{-1}$ with an error on the mean of $0.19 \mathrm{~km} \mathrm{~s}^{-1}$. Normally, a $\sim 5 \mathrm{~km} \mathrm{~s}^{-1}$ difference between epochs would indicate a binary (assuming the uncertainties are less) since the offset is apparent in most of the sources with high $R$ values. That is not the case for these specific objects. In contrast, for the objects that were originally observed in 2010 or 2011 and observed again in 2013, a weighted offset of $0.1 \mathrm{~km} \mathrm{~s}^{-1}$ was found, with an error on the mean of $0.1 \mathrm{~km} \mathrm{~s}^{-1}$. This offset is easily within the uncertainties of the data, and therefore, no offset correction was needed for the 2010 and 2011 epochs. The $5.5 \mathrm{~km} \mathrm{~s}^{-1}$ offset was applied to both 2009 epochs and the uncertainty of the offset was combined in quadrature with the velocity uncertainties of each object in the 2009 data. These corrected values and the combined uncertainties are used in the tables and analysis.

The RV measurements of all observations are listed in Tables 2-6. The origin of this offset remains unclear. The uncertainties obtained from our analysis are consistent with previous results from Hectoschelle (Fúrész et al. 2006, 2008; Tobin et al. 2009, 2015; Kounkel et al. 2017).

\subsection{Identifying Binaries}

Potential spectroscopic binaries are listed in Table 7. Binaries of similar mass give a double-peak correlation plot, which was seen in 15 objects. For mass ratios less than unity, the primary component will dominate the spectrum and thus the RV will measure the motion of the primary. The secondary component may contaminate the spectrum enough to add an asymmetry. A total of 39 objects with significant asymmetries in their cross-correlation curves were also marked as potential binaries. If a source was observed twice, we compared the RVs to search for additional binaries. A shift in RV could be noticeable for short- to moderate-period binaries, i.e., orbital periods ranging from weeks up to $\sim 10 \mathrm{yr}$. For objects with no companion, the RV should remain the same within the uncertainties. Of the 64 objects observed for a second time, 
Table 3

2009.1103 RV Results

\begin{tabular}{|c|c|c|c|c|}
\hline 2MASS ID & R.A. (J2000) & Decl. (J2000) & $\overline{\mathrm{RV}} \pm \sigma\left(\mathrm{km} \mathrm{s}^{-1}\right)$ & $\overline{R \text { Value }}$ \\
\hline $\mathrm{J} 22535500+6243412$ & $22: 53: 55.00$ & $62: 43: 41.24$ & $-66.96 \pm 4.06$ & 14.79 \\
\hline $\mathrm{J} 22542631+6242377$ & $22: 54: 26.33$ & $62: 42: 37.77$ & $-10.37 \pm 1.73$ & 8.47 \\
\hline $\mathrm{J} 22542993+6240335$ & $22: 54: 29.92$ & $62: 40: 33.57$ & $-8.10 \pm 7.02$ & 2.21 \\
\hline $\mathrm{J} 22535448+6244536$ & $22: 53: 54.47$ & 62:44:53.72 & $-18.34 \pm 4.24$ & 1.43 \\
\hline $\mathrm{J} 22541764+6248194$ & $22: 54: 17.64$ & $62: 48: 19.40$ & $-28.77 \pm 1.54$ & 6.33 \\
\hline $\mathrm{J} 22542450+6245330$ & $22: 54: 24.51$ & $62: 45: 32.96$ & $-23.56 \pm 1.54$ & 7.31 \\
\hline $\mathrm{J} 22540417+6247032$ & $22: 54: 04.16$ & 62:47:03.23 & $-16.08 \pm 3.71$ & 2.90 \\
\hline $\mathrm{J} 22543463+6242480$ & $22: 54: 34.62$ & 62:42:47.99 & $-51.03 \pm 7.64$ & 12.54 \\
\hline $\mathrm{J} 22545800+6243449$ & $22: 54: 58.03$ & $62: 43: 44.95$ & $-30.67 \pm 1.00$ & 10.49 \\
\hline $\mathrm{J} 22544726+6249150$ & $22: 54: 47.28$ & 62:49:15.14 & $0.19 \pm 6.30$ & 2.02 \\
\hline $\mathrm{J} 22543337+6256079$ & $22: 54: 33.36$ & 62:56:07.82 & $-25.93 \pm 11.50$ & 1.78 \\
\hline $\mathrm{J} 22541872+6251459$ & $22: 54: 18.72$ & 62:51:45.89 & $-11.51 \pm 3.50$ & 0.67 \\
\hline $\mathrm{J} 22543667+6249120$ & $22: 54: 36.68$ & 62:49:11.94 & $-3.71 \pm 4.39$ & 7.03 \\
\hline $\mathrm{J} 22543513+6245431$ & $22: 54: 35.14$ & $62: 45: 43.20$ & $-39.69 \pm 7.02$ & 2.54 \\
\hline $\mathrm{J} 22542604+6255213$ & $22: 54: 26.06$ & $62: 55: 21.21$ & $-13.39 \pm 1.06$ & 9.74 \\
\hline $\mathrm{J} 22551415+6255523$ & $22: 55: 14.13$ & $62: 55: 52.37$ & $-36.43 \pm 0.77$ & 14.45 \\
\hline $\mathrm{J} 22550275+6241499$ & $22: 55: 02.73$ & 62:41:49.95 & $-18.19 \pm 4.34$ & 1.64 \\
\hline $\mathrm{J} 22550915+6253570$ & $22: 55: 09.14$ & 62:53:57.12 & $-31.49 \pm 7.90$ & 3.86 \\
\hline $\mathrm{J} 22550636+6256529$ & $22: 55: 06.36$ & 62:56:52.85 & $-27.91 \pm 1.54$ & 7.06 \\
\hline $\mathrm{J} 22553178+6246430$ & $22: 55: 31.82$ & $62: 46: 43.33$ & $-22.70 \pm 0.89$ & 11.93 \\
\hline $\mathrm{J} 22554366+6250388$ & $22: 55: 43.67$ & $62: 50: 38.76$ & $-10.54 \pm 2.18$ & 3.46 \\
\hline $\mathrm{J} 22552486+6246446$ & $22: 55: 24.87$ & $62: 46: 44.63$ & $33.22 \pm 3.34$ & 2.26 \\
\hline $\mathrm{J} 22553847+6255182$ & $22: 55: 38.46$ & $62: 55: 18.17$ & $-42.05 \pm 5.32$ & 0.56 \\
\hline $\mathrm{J} 22553242+6250527$ & $22: 55: 32.39$ & $62: 50: 52.65$ & $-75.95 \pm 3.34$ & 5.16 \\
\hline $\mathrm{J} 22553628+6246094$ & $22: 55: 36.27$ & 62:46:09.56 & $-81.11 \pm 3.17$ & 16.75 \\
\hline $\mathrm{J} 22553545+6242343$ & $22: 55: 35.44$ & $62: 42: 34.38$ & $-36.85 \pm 1.26$ & 8.69 \\
\hline $\mathrm{J} 22560150+6247467$ & $22: 56: 01.48$ & $62: 47: 46.73$ & $-14.46 \pm 2.22$ & 5.12 \\
\hline $\mathrm{J} 22560924+6250324$ & $22: 56: 09.98$ & 62:50:13.96 & $12.32 \pm 3.88$ & 0.94 \\
\hline $\mathrm{J} 22560894+6251154$ & $22: 56: 08.98$ & $62: 51: 15.49$ & $-11.26 \pm 1.15$ & 9.01 \\
\hline $\mathrm{J} 22562585+6253570$ & $22: 56: 25.85$ & 62:53:57.09 & $-51.51 \pm 3.38$ & 9.36 \\
\hline $\mathrm{J} 22561473+6247246$ & $22: 56: 14.73$ & $62: 47: 24.72$ & $-12.46 \pm 1.54$ & 6.40 \\
\hline $\mathrm{J} 22564512+6246193$ & $22: 56: 45.12$ & $62: 46: 19.28$ & $-12.09 \pm 1.66$ & 6.74 \\
\hline $\mathrm{J} 22560766+6246022$ & $22: 56: 07.64$ & $62: 46: 02.33$ & $-9.63 \pm 4.18$ & 0.94 \\
\hline $\mathrm{J} 22562963+6245284$ & $22: 56: 29.63$ & $62: 45: 28.52$ & $-10.77 \pm 1.55$ & 9.45 \\
\hline $\mathrm{J} 22570195+6251053$ & $22: 57: 01.94$ & 62:51:05.45 & $-34.10 \pm 0.67$ & 15.18 \\
\hline $\mathrm{J} 22563105+6246581$ & $22: 56: 31.04$ & 62:46:58.19 & $-13.48 \pm 1.04$ & 10.82 \\
\hline $\mathrm{J} 22554749+6241576$ & $22: 55: 47.49$ & 62:41:57.68 & $-11.94 \pm 4.73$ & 3.12 \\
\hline $\mathrm{J} 22573150+6243576$ & $22: 57: 31.53$ & $62: 43: 57.58$ & $-15.16 \pm 2.26$ & 3.80 \\
\hline $\mathrm{J} 22564371+6242017$ & $22: 56: 43.72$ & 62:42:01.68 & $-12.56 \pm 5.08$ & 3.12 \\
\hline $\mathrm{J} 22562860+6244405$ & $22: 56: 28.57$ & 62:44:40.49 & $-11.17 \pm 1.63$ & 6.46 \\
\hline $\mathrm{J} 22563590+6242057$ & $22: 56: 35.89$ & $62: 42: 05.52$ & $29.80 \pm 3.53$ & 2.96 \\
\hline $\mathrm{J} 22560428+6242388$ & $22: 56: 04.27$ & 62:42:38.89 & $26.53 \pm 12.40$ & 2.19 \\
\hline $\mathrm{J} 22565123+6243197$ & $22: 56: 51.24$ & $62: 43: 19.74$ & $-18.12 \pm 2.77$ & 5.80 \\
\hline $\mathrm{J} 22561518+6242475$ & $22: 56: 15.14$ & $62: 42: 47.35$ & $-12.90 \pm 2.01$ & 4.30 \\
\hline $\mathrm{J} 22562819+6242463$ & $22: 56: 28.19$ & 62:42:46.32 & $-32.09 \pm 1.22$ & 7.96 \\
\hline $\mathrm{J} 22564619+6244225$ & $22: 56: 46.17$ & $62: 44: 22.73$ & $-14.92 \pm 1.77$ & 5.77 \\
\hline $\mathrm{J} 22570594+6238180$ & $22: 57: 05.95$ & $62: 38: 18.05$ & $-13.51 \pm 2.08$ & 5.52 \\
\hline $\mathrm{J} 22562231+6240488$ & $22: 56: 22.31$ & 62:40:48.92 & $-10.51 \pm 1.65$ & 6.71 \\
\hline $\mathrm{J} 22570529+6238332$ & $22: 57: 05.18$ & $62: 38: 38.57$ & $-39.06 \pm 1.15$ & 8.38 \\
\hline $\mathrm{J} 22563259+6238417$ & $22: 56: 32.57$ & 62:38:41.71 & $-26.41 \pm 14.55$ & 0.68 \\
\hline $\mathrm{J} 22560480+6239460$ & $22: 56: 04.81$ & 62:39:46.22 & $-10.67 \pm 1.08$ & 9.19 \\
\hline $\mathrm{J} 22563527+6239074$ & $22: 56: 35.28$ & 62:39:07.57 & $-12.91 \pm 2.21$ & 5.21 \\
\hline $\mathrm{J} 22563654+6240077$ & $22: 56: 36.55$ & $62: 40: 07.52$ & $-61.53 \pm 4.64$ & 7.45 \\
\hline $\mathrm{J} 22562780+6239282$ & $22: 56: 27.82$ & $62: 39: 28.24$ & $36.37 \pm 29.47$ & 2.43 \\
\hline $\mathrm{J} 22564296+6235316$ & $22: 56: 42.94$ & $62: 35: 31.62$ & $-17.21 \pm 3.30$ & 1.62 \\
\hline $\mathrm{J} 22571485+6237437$ & $22: 57: 14.85$ & $62: 37: 43.82$ & $-5.85 \pm 0.95$ & 10.78 \\
\hline $\mathrm{J} 22572343+6235141$ & $22: 57: 23.43$ & 62:35:14.10 & $-8.71 \pm 2.86$ & 3.37 \\
\hline $\mathrm{J} 22563865+6237140$ & $22: 56: 38.68$ & $62: 37: 14.29$ & $-31.93 \pm 4.53$ & 3.83 \\
\hline $\mathrm{J} 22564210+6236207$ & $22: 56: 42.11$ & $62: 36: 20.88$ & $-19.76 \pm 1.90$ & 5.26 \\
\hline $\mathrm{J} 22560823+6237034$ & $22: 56: 08.23$ & 62:37:03.54 & $-32.23 \pm 1.26$ & 10.12 \\
\hline $\mathrm{J} 22571812+6237011$ & $22: 57: 18.13$ & 62:37:00.98 & $-26.21 \pm 1.25$ & 8.60 \\
\hline J22570709+6230189 & $22: 57: 07.08$ & 62:30:19.05 & $-21.59 \pm 2.85$ & 3.84 \\
\hline $\mathrm{J} 22560306+6235187$ & $22: 56: 03.04$ & $62: 35: 18.71$ & $-24.33 \pm 4.23$ & 1.88 \\
\hline $\mathrm{J} 22553832+6235472$ & $22: 55: 38.32$ & $62: 35: 47.31$ & $3.00 \pm 0.19$ & $\ldots$ \\
\hline $\mathrm{J} 22562274+6232501$ & $22: 56: 22.76$ & 62:32:50.11 & $-16.13 \pm 8.07$ & 1.54 \\
\hline
\end{tabular}


Table 3

(Continued)

\begin{tabular}{|c|c|c|c|c|}
\hline 2MASS ID & R.A. (J2000) & Decl. (J2000) & $\overline{\mathrm{RV}} \pm \sigma\left(\mathrm{km} \mathrm{s}^{-1}\right)$ & $\overline{R \text { Value }}$ \\
\hline $\mathrm{J} 225556866+6234332$ & $22: 55: 56.85$ & $62: 34: 33.24$ & $-12.89 \pm 2.72$ & 5.42 \\
\hline $\mathrm{J} 22565217+6226391$ & $22: 56: 52.17$ & $62: 26: 39.06$ & $2.97 \pm 0.19$ & $\ldots$ \\
\hline $\mathrm{J} 22570845+6225441$ & 22:57:08.44 & $62: 25: 44.28$ & $2.96 \pm 0.19$ & $\cdots$ \\
\hline $\mathrm{J} 22565569+6229183$ & $22: 56: 55.67$ & $62: 29: 18.36$ & $2.97 \pm 0.19$ & $\ldots$ \\
\hline $\mathrm{J} 22563710+6229456$ & $22: 56: 37.40$ & $62: 29: 46.40$ & $33.69 \pm 1.38$ & 7.01 \\
\hline $\mathrm{J} 22571116+6227330$ & $22: 57: 11.15$ & $62: 27: 33.00$ & $43.02 \pm 9.30$ & 1.03 \\
\hline $\mathrm{J} 22562181+6223029$ & $22: 56: 21.84$ & $62: 23: 02.95$ & $-12.96 \pm 0.85$ & 12.99 \\
\hline $\mathrm{J} 22553297+6233412$ & $22: 55: 32.96$ & $62: 33: 41.27$ & $3.01 \pm 0.19$ & $\ldots$ \\
\hline $\mathrm{J} 22552589+6232538$ & $22: 55: 25.89$ & $62: 32: 53.85$ & $-4.86 \pm 1.58$ & 6.67 \\
\hline $\mathrm{J} 22551567+6235261$ & $22: 55: 15.67$ & $62: 35: 26.16$ & $-5.71 \pm 11.37$ & 1.96 \\
\hline $\mathrm{J} 22545580+6236233$ & 22:54:55.94 & $62: 36: 23.34$ & $3.02 \pm 0.19$ & $\ldots$ \\
\hline $\mathrm{J} 22560310+6225197$ & 22:56:03.12 & $62: 25: 19.82$ & $5.53 \pm 1.68$ & 6.99 \\
\hline $\mathrm{J} 22555006+6223083$ & $22: 55: 50.15$ & $62: 23: 08.25$ & $-16.35 \pm 4.04$ & 5.42 \\
\hline $\mathrm{J} 22551657+6232522$ & $22: 55: 16.59$ & $62: 32: 52.12$ & $-14.09 \pm 3.57$ & 3.02 \\
\hline $\mathrm{J} 22550265+6229136$ & $22: 55: 02.64$ & $62: 29: 13.61$ & $-1.85 \pm 6.28$ & 1.16 \\
\hline $\mathrm{J} 22551869+6223517$ & $22: 55: 18.70$ & $62: 23: 51.79$ & $-82.85 \pm 5.58$ & 15.92 \\
\hline $\mathrm{J} 22544780+6228506$ & $22: 54: 47.81$ & $62: 28: 50.46$ & $34.37 \pm 5.48$ & 0.98 \\
\hline $\mathrm{J} 22545480+6233514$ & $22: 54: 55.12$ & $62: 33: 40.13$ & $-27.72 \pm 1.32$ & 7.47 \\
\hline $\mathrm{J} 22545176+6230337$ & $22: 54: 51.76$ & $62: 30: 33.50$ & $-43.55 \pm 1.52$ & 6.38 \\
\hline $\mathrm{J} 22544036+6233335$ & $22: 54: 40.36$ & $62: 33: 33.45$ & $-9.52 \pm 1.04$ & 9.89 \\
\hline $\mathrm{J} 22544382+6230376$ & $22: 54: 43.81$ & $62: 30: 37.83$ & $3.03 \pm 0.19$ & $\ldots$ \\
\hline $\mathrm{J} 22542760+6225270$ & $22: 54: 27.60$ & $62: 25: 26.87$ & $-2.58 \pm 3.83$ & 3.13 \\
\hline $\mathrm{J} 22540555+6223064$ & 22:54:05.54 & $62: 23: 06.65$ & $-29.98 \pm 1.08$ & 10.04 \\
\hline $\mathrm{J} 22542257+6225041$ & $22: 54: 22.56$ & $62: 25: 04.02$ & $-12.37 \pm 3.43$ & 4.60 \\
\hline $\mathrm{J} 22541824+6226460$ & $22: 54: 18.24$ & $62: 26: 45.93$ & $-5.13 \pm 8.07$ & 1.31 \\
\hline $\mathrm{J} 22544224+6235548$ & $22: 54: 42.23$ & $62: 35: 54.93$ & $-9.86 \pm 1.97$ & 5.38 \\
\hline $\mathrm{J} 22544431+6235183$ & $22: 54: 44.31$ & $62: 35: 18.23$ & $10.50 \pm 0.96$ & 11.75 \\
\hline $\mathrm{J} 22542545+6234245$ & $22: 54: 25.47$ & $62: 34: 24.59$ & $-14.97 \pm 1.41$ & 8.12 \\
\hline $\mathrm{J} 22530590+6225392$ & $22: 53: 05.90$ & $62: 25: 39.02$ & $-22.23 \pm 0.76$ & 14.61 \\
\hline $\mathrm{J} 22522558+6222059$ & $22: 52: 25.60$ & 62:22:05.91 & $-14.94 \pm 6.56$ & 2.64 \\
\hline $\mathrm{J} 22531582+6230219$ & $22: 53: 15.81$ & $62: 30: 22.14$ & $17.50 \pm 1.36$ & 7.28 \\
\hline $\mathrm{J} 22530014+6230588$ & $22: 53: 01.67$ & $62: 30: 55.69$ & $4.78 \pm 0.94$ & 10.75 \\
\hline $\mathrm{J} 22525242+6228599$ & $22: 52: 52.45$ & $62: 28: 59.95$ & $-3.58 \pm 4.95$ & 2.18 \\
\hline $\mathrm{J} 22543423+6235555$ & $22: 54: 34.20$ & $62: 35: 55.46$ & $2.70 \pm 5.57$ & 3.04 \\
\hline $\mathrm{J} 22535395+6234504$ & $22: 53: 53.93$ & $62: 34: 50.29$ & $-12.78 \pm 2.69$ & 2.19 \\
\hline $\mathrm{J} 22540214+6236278$ & 22:54:02.16 & $62: 36: 27.78$ & $-18.07 \pm 4.50$ & 2.86 \\
\hline $\mathrm{J} 22540337+6235322$ & $22: 54: 03.39$ & $62: 35: 32.25$ & $-73.14 \pm 3.68$ & 15.91 \\
\hline $\mathrm{J} 22531578+6235262$ & $22: 53: 15.78$ & $62: 35: 26.13$ & $-12.71 \pm 15.25$ & 2.30 \\
\hline $\mathrm{J} 22521616+6233017$ & $22: 52: 16.16$ & $62: 33: 01.65$ & $-13.49 \pm 1.79$ & 7.34 \\
\hline $\mathrm{J} 22532187+6234490$ & $22: 53: 21.86$ & $62: 34: 48.95$ & $-13.34 \pm 4.93$ & 1.06 \\
\hline $\mathrm{J} 22525910+6239184$ & $22: 52: 59.12$ & $62: 39: 18.55$ & $1.37 \pm 0.93$ & 11.58 \\
\hline $\mathrm{J} 22533664+6236489$ & $22: 53: 36.65$ & $62: 36: 48.99$ & $-13.82 \pm 7.32$ & 3.85 \\
\hline $\mathrm{J} 22523061+6238279$ & $22: 52: 33.08$ & $62: 38: 48.02$ & $-71.28 \pm 4.49$ & 17.09 \\
\hline $\mathrm{J} 22533869+6237171$ & $22: 53: 38.67$ & $62: 37: 17.23$ & $11.21 \pm 7.64$ & 2.31 \\
\hline $\mathrm{J} 22534042+6237564$ & $22: 53: 40.40$ & $62: 37: 56.44$ & $3.57 \pm 2.10$ & 4.03 \\
\hline $\mathrm{J} 22532277+6237340$ & $22: 53: 22.77$ & $62: 37: 34.07$ & $-11.14 \pm 1.88$ & 5.31 \\
\hline $\mathrm{J} 22524772+6240044$ & $22: 52: 47.70$ & $62: 40: 04.72$ & $-16.89 \pm 3.81$ & 2.85 \\
\hline $\mathrm{J} 22544247+6237542$ & $22: 54: 42.44$ & $62: 37: 54.36$ & $-18.86 \pm 9.65$ & 1.21 \\
\hline $\mathrm{J} 22522353+6244227$ & $22: 52: 23.53$ & $62: 44: 22.62$ & $-44.65 \pm 0.93$ & 11.60 \\
\hline $\mathrm{J} 22525120+6241051$ & $22: 52: 51.21$ & $62: 41: 05.14$ & $40.79 \pm 3.43$ & 1.97 \\
\hline $\mathrm{J} 22533793+6240148$ & $22: 53: 37.94$ & $62: 40: 14.86$ & $-7.04 \pm 1.01$ & 10.77 \\
\hline $\mathrm{J} 22540382+6238211$ & $22: 54: 03.84$ & $62: 38: 21.03$ & $-12.27 \pm 1.15$ & 9.32 \\
\hline $\mathrm{J} 22541076+6241389$ & $22: 54: 10.75$ & $62: 41: 38.90$ & $-2.02 \pm 4.62$ & 1.93 \\
\hline $\mathrm{J} 22525607+6244047$ & $22: 52: 56.06$ & $62: 44: 04.73$ & $-4.92 \pm 7.85$ & 1.53 \\
\hline $\mathrm{J} 22533684+6243149$ & $22: 53: 36.83$ & $62: 43: 14.88$ & $-43.15 \pm 1.64$ & 5.90 \\
\hline $\mathrm{J} 22525172+6244534$ & $22: 52: 50.98$ & $62: 44: 46.91$ & $32.27 \pm 5.91$ & 0.72 \\
\hline $\mathrm{J} 22540533+6240313$ & $22: 54: 05.31$ & $62: 40: 31.33$ & $-14.72 \pm 6.10$ & 1.18 \\
\hline $\mathrm{J} 22534960+6241093$ & $22: 53: 49.58$ & $62: 41: 09.42$ & $-11.89 \pm 4.60$ & 1.17 \\
\hline
\end{tabular}

34 disagree at the $>1 \sigma$ level and 21 disagree at the $>2 \sigma$ level. It is expected that at least 20 objects would differ at the $1 \sigma$ level even if none are binaries; the larger number of objects in disagreement suggests a short-period binary frequency of $\sim 20 \%$ in our sample. We identify all of the $>1 \sigma$ objects as potential binaries. 
Table 4

2010 RV Results

\begin{tabular}{|c|c|c|c|c|}
\hline 2MASS ID & R.A. (J2000) & Decl. (J2000) & $\mathrm{RV} \pm \sigma\left(\mathrm{km} \mathrm{s}^{-1}\right)$ & $R$ Value \\
\hline $\mathrm{J} 22542652+6247077$ & $22: 54: 26.50$ & $62: 47: 07.79$ & $39.49 \pm 5.24$ & 0.99 \\
\hline J22543806+6251022 & 22:54:38.06 & $62: 51: 02.27$ & $-38.54 \pm 1.13$ & 9.35 \\
\hline $\mathrm{J} 22542386+6255382$ & $22: 54: 22.92$ & $62: 55: 24.16$ & $40.46 \pm 3.22$ & 1.65 \\
\hline $\mathrm{J} 22543139+6251547$ & $22: 54: 31.39$ & $62: 51: 54.54$ & $4.47 \pm 2.09$ & 4.50 \\
\hline $\mathrm{J} 22542604+6255213$ & $22: 54: 27.90$ & $62: 55: 26.17$ & $50.14 \pm 4.53$ & 1.77 \\
\hline $\mathrm{J} 22545426+6254405$ & $22: 54: 54.24$ & $62: 54: 40.48$ & $-105.70 \pm 0.73$ & 14.22 \\
\hline $\mathrm{J} 22551602+6255125$ & $22: 55: 15.89$ & $62: 55: 21.30$ & $-36.91 \pm 4.06$ & 1.85 \\
\hline $\mathrm{J} 22550634+6253203$ & $22: 55: 06.26$ & $62: 53: 20.52$ & $-39.92 \pm 1.46$ & 6.96 \\
\hline $\mathrm{J} 22550859+6255151$ & $22: 55: 07.83$ & $62: 55: 21.52$ & $-41.43 \pm 3.21$ & 1.92 \\
\hline $\mathrm{J} 22550760+6244452$ & 22:55:08.12 & $62: 44: 57.93$ & $35.31 \pm 2.45$ & 3.53 \\
\hline $\mathrm{J} 22552454+6254456$ & $22: 55: 24.52$ & $62: 54: 45.70$ & $-9.71 \pm 1.53$ & 6.16 \\
\hline $\mathrm{J} 22553086+6252057$ & $22: 55: 30.86$ & $62: 52: 05.64$ & $-33.64 \pm 0.73$ & 14.69 \\
\hline $\mathrm{J} 22553847+6255182$ & $22: 55: 37.59$ & $62: 55: 19.18$ & $49.39 \pm 7.66$ & 1.06 \\
\hline $\mathrm{J} 22553359+6253287$ & $22: 55: 33.59$ & $62: 53: 28.89$ & $-12.82 \pm 1.54$ & 6.38 \\
\hline $\mathrm{J} 22555591+6252385$ & $22: 55: 55.86$ & $62: 52: 38.46$ & $-15.80 \pm 7.92$ & 2.22 \\
\hline $\mathrm{J} 22561016+6249328$ & $22: 56: 10.18$ & $62: 49: 32.87$ & $-21.10 \pm 5.64$ & 1.60 \\
\hline $\mathrm{J} 22562031+6254458$ & $22: 56: 20.39$ & $62: 54: 46.05$ & $-17.91 \pm 1.62$ & 7.73 \\
\hline $\mathrm{J} 22571752+6256356$ & $22: 57: 17.52$ & $62: 56: 35.50$ & $-51.19 \pm 1.15$ & 9.13 \\
\hline $\mathrm{J} 22562450+6254314$ & $22: 56: 24.50$ & $62: 54: 31.45$ & $33.18 \pm 1.27$ & 8.09 \\
\hline $\mathrm{J} 22550959+6244210$ & 22:55:09.61 & $62: 44.21 .04$ & $-7.00 \pm 4.12$ & 5.29 \\
\hline $\mathrm{J} 22551755+6245458$ & $22: 55: 17.53$ & $62: 45: 45.94$ & $-6.07 \pm 3.64$ & 5.28 \\
\hline $\mathrm{J} 22555228+6249200$ & $22: 55: 52.27$ & $62: 49: 20.03$ & $-6.01 \pm 1.21$ & 8.12 \\
\hline $\mathrm{J} 22560030+6245029$ & $22: 56: 00.31$ & 62:45:03.05 & $-11.82 \pm 0.91$ & 11.57 \\
\hline $\mathrm{J} 22545157+6241522$ & $22: 54: 51.54$ & $62: 41: 52.35$ & $-43.61 \pm 0.89$ & 11.97 \\
\hline $\mathrm{J} 22565619+6248492$ & $22: 56: 56.22$ & $62: 48: 49.24$ & $-37.19 \pm 0.65$ & 16.50 \\
\hline $\mathrm{J} 22564191+6250549$ & 22:56:41.91 & $62: 50: 54.96$ & $23.15 \pm 7.62$ & 0.74 \\
\hline $\mathrm{J} 22560068+6245310$ & $22: 56: 00.68$ & $62: 45: 30.95$ & $-9.33 \pm 1.59$ & 5.70 \\
\hline $\mathrm{J} 22551760+6243231$ & $22: 55: 17.61$ & 62:43:22.99 & $-9.33 \pm 2.84$ & 6.56 \\
\hline $\mathrm{J} 22561978+6248022$ & 22:56:19.81 & $62: 48: 02.17$ & $-2.35 \pm 3.75$ & 3.78 \\
\hline $\mathrm{J} 22563678+6246031$ & $22: 56: 36.82$ & $62: 46: 02.94$ & $-8.02 \pm 7.08$ & 3.83 \\
\hline $\mathrm{J} 22572663+6243491$ & $22: 57: 26.64$ & $62: 43: 49.15$ & $-14.69 \pm 1.06$ & 11.05 \\
\hline $\mathrm{J} 22560391+6243359$ & 22:56:03.89 & $62: 43: 35.96$ & $-11.12 \pm 1.18$ & 8.41 \\
\hline $\mathrm{J} 22562634+6242414$ & $22: 56: 26.32$ & $62: 42: 41.44$ & $-13.29 \pm 1.32$ & 10.39 \\
\hline $\mathrm{J} 22565515+6245088$ & $22: 56: 55.15$ & $62: 45: 08.83$ & $-12.75 \pm 3.35$ & 4.05 \\
\hline $\mathrm{J} 22561924+6242528$ & $22: 56: 19.22$ & $62: 42: 52.66$ & $-4.74 \pm 1.08$ & 9.48 \\
\hline $\mathrm{J} 22570981+6245125$ & 22:57:06.71 & 62:45:08.09 & $-66.04 \pm 0.82$ & 11.73 \\
\hline $\mathrm{J} 22562134+6243257$ & $22: 56: 21.33$ & $62: 43: 25.71$ & $-12.87 \pm 3.89$ & 6.73 \\
\hline $\mathrm{J} 22583320+6247456$ & $22: 58: 33.21$ & $62: 47: 45.65$ & $-9.05 \pm 14.90$ & 1.69 \\
\hline $\mathrm{J} 22555158+6239116$ & $22: 55: 51.55$ & $62: 39: 11.78$ & $-8.56 \pm 1.04$ & 10.55 \\
\hline $\mathrm{J} 22565035+6242485$ & $22: 56: 50.36$ & $62: 42: 48.58$ & $-15.15 \pm 4.60$ & 1.87 \\
\hline J22570584+6239222 & 22:57:06.00 & $62: 39: 26.19$ & $-32.25 \pm 0.92$ & 11.69 \\
\hline $\mathrm{J} 22570134+6242133$ & $22: 57: 01.36$ & $62: 42: 13.43$ & $-12.87 \pm 13.56$ & 2.14 \\
\hline $\mathrm{J} 22561018+6239418$ & $22: 56: 10.18$ & $62: 39: 41.82$ & $-10.93 \pm 3.73$ & 2.29 \\
\hline $\mathrm{J} 22551119+6239142$ & $22: 55: 11.99$ & $62: 40: 37.34$ & $-11.25 \pm 1.47$ & 6.50 \\
\hline $\mathrm{J} 22555016+6240070$ & $22: 55: 50.17$ & $62: 40: 07.24$ & $-9.27 \pm 0.99$ & 12.59 \\
\hline $\mathrm{J} 22563127+6240588$ & $22: 56: 31.26$ & $62: 40: 58.71$ & $-11.92 \pm 4.30$ & 3.89 \\
\hline $\mathrm{J} 22554485+6237199$ & $22: 55: 44.84$ & $62: 37: 19.82$ & $-10.05 \pm 14.08$ & 0.94 \\
\hline $\mathrm{J} 22564720+6237055$ & $22: 56: 47.20$ & $62: 37: 05.34$ & $-10.26 \pm 3.57$ & 4.22 \\
\hline J22560969+6237289 & 22:56:09.69 & $62: 37: 29.05$ & $-16.30 \pm 6.33$ & 0.54 \\
\hline J22544942+6239227 & $22: 54: 49.40$ & $62: 39: 22.60$ & $-9.59 \pm 1.55$ & 6.85 \\
\hline $\mathrm{J} 22560648+6234430$ & $22: 56: 06.49$ & $62: 34: 43.15$ & $-30.00 \pm 1.06$ & 10.11 \\
\hline $\mathrm{J} 22565314+6232094$ & $22: 56: 53.15$ & 62:32:09.41 & $21.28 \pm 1.02$ & 10.89 \\
\hline $\mathrm{J} 22560790+6230425$ & 22:56:07.90 & $62: 30: 42.56$ & $-12.82 \pm 2.54$ & 3.90 \\
\hline $\mathrm{J} 22560767+6221482$ & $22: 56: 07.72$ & $62: 21: 48.07$ & $-21.15 \pm 4.92$ & 1.45 \\
\hline $\mathrm{J} 22545509+6234545$ & $22: 54: 55.07$ & $62: 34: 54.41$ & $25.39 \pm 15.02$ & 1.34 \\
\hline $\mathrm{J} 22544057+6238097$ & $22: 54: 40.54$ & 62:38:09.59 & $-3.91 \pm 6.45$ & 1.38 \\
\hline $\mathrm{J} 22542460+6225053$ & $22: 54: 24.63$ & $62: 25: 05: 22$ & $-25.93 \pm 1.95$ & 5.08 \\
\hline $\mathrm{J} 22543310+6233339$ & $22: 54: 33.11$ & $62: 33: 33.84$ & $5.68 \pm 2.11$ & 4.98 \\
\hline $\mathrm{J} 22543924+6233469$ & $22: 54: 39.25$ & $62: 33: 46.79$ & $-9.21 \pm 6.29$ & 0.62 \\
\hline $\mathrm{J} 22543166+6237117$ & $22: 54: 31.65$ & $62: 37: 11.78$ & $-10.61 \pm 2.60$ & 3.48 \\
\hline $\mathrm{J} 22525674+6223004$ & $22: 52: 56.72$ & 62:23:00.49 & $-0.22 \pm 1.46$ & 7.16 \\
\hline $\mathrm{J} 22542236+6236471$ & $22: 54: 22.35$ & $62: 36: 47.25$ & $-69.99 \pm 0.91$ & 10.54 \\
\hline $\mathrm{J} 22541270+6235379$ & $22: 54: 12.70$ & $62: 35: 37.89$ & $-40.86 \pm 2.86$ & 3.02 \\
\hline $\mathrm{J} 22535945+6235102$ & $22: 53: 59.42$ & $62: 35: 10.21$ & $53.78 \pm 3.16$ & 2.10 \\
\hline
\end{tabular}


Table 4

(Continued)

\begin{tabular}{|c|c|c|c|c|}
\hline 2MASS ID & R.A. (J2000) & Decl. (J2000) & $\mathrm{RV} \pm \sigma\left(\mathrm{km} \mathrm{s}^{-1}\right)$ & $R$ Value \\
\hline $\mathrm{J} 22535969+6236320$ & 22:53:59.69 & $62: 36: 32.03$ & $-22.27 \pm 4.59$ & 4.66 \\
\hline $\mathrm{J} 22523376+6224147$ & $22: 52: 33.75$ & $62: 24: 14.71$ & $0.55 \pm 1.96$ & 5.24 \\
\hline $\mathrm{J} 22534194+6234103$ & $22: 53: 43.50$ & $62: 34: 17.49$ & $-44.68 \pm 0.69$ & 15.29 \\
\hline $\mathrm{J} 22535011+6234322$ & $22: 53: 50.10$ & $62: 34: 32.30$ & $44.35 \pm 8.66$ & 0.74 \\
\hline $\mathrm{J} 22540194+6238255$ & 22:54:01.92 & $62: 38: 25.45$ & $-7.61 \pm 2.58$ & 2.85 \\
\hline $\mathrm{J} 22525102+6230436$ & $22: 52: 51.00$ & $62: 30: 43.51$ & $3.00 \pm 1.33$ & 7.63 \\
\hline $\mathrm{J} 22531574+6234357$ & $22: 53: 15.71$ & $62: 34: 35.77$ & $-41.03 \pm 3.84$ & 2.79 \\
\hline $\mathrm{J} 22524793+6230571$ & $22: 52: 47.92$ & $62: 30: 57.18$ & $10.12 \pm 1.71$ & 6.00 \\
\hline $\mathrm{J} 22542708+6238531$ & $22: 54: 27.07$ & $62: 38: 53.06$ & $-26.34 \pm 5.11$ & 1.27 \\
\hline $\mathrm{J} 22531015+6236444$ & $22: 53: 10.17$ & $62: 36: 44.41$ & $-17.44 \pm 3.09$ & 4.60 \\
\hline $\mathrm{J} 22504792+6233227$ & 22:50:47.94 & $62: 33: 22.62$ & $-33.24 \pm 2.58$ & 4.14 \\
\hline $\mathrm{J} 22530436+6237249$ & $22: 53: 04.38$ & $62: 37: 24.91$ & $-7.69 \pm 4.63$ & 1.93 \\
\hline $\mathrm{J} 22532653+6240134$ & $22: 53: 26.53$ & $62: 40: 13.57$ & $-8.45 \pm 1.42$ & 7.73 \\
\hline $\mathrm{J} 22531297+6238444$ & $22: 53: 12.99$ & $62: 38: 44.39$ & $-31.07 \pm 1.50$ & 7.34 \\
\hline $\mathrm{J} 02530517+6239073$ & $22: 53: 05.03$ & 62:39:09.61 & $-21.69 \pm 5.38$ & 2.34 \\
\hline $\mathrm{J} 22523412+6241513$ & $22: 52: 34.10$ & $62: 41: 51.26$ & $-26.78 \pm 1.03$ & 10.76 \\
\hline $\mathrm{J} 22530099+6243548$ & 22:53:01.01 & $62: 43: 54.70$ & $-19.18 \pm 6.82$ & 2.97 \\
\hline $\mathrm{J} 22535397+6240249$ & $22: 53: 53.95$ & $62: 40: 24.90$ & $-80.14 \pm 1.28$ & 8.08 \\
\hline $\mathrm{J} 22523028+6243087$ & $22: 52: 30.31$ & $62: 43: 08.63$ & $42.75 \pm 1.79$ & 0.03 \\
\hline $\mathrm{J} 22532070+6242187$ & $22: 53: 20.70$ & $62: 42: 18.83$ & $-11.24 \pm 3.06$ & 5.50 \\
\hline $\mathrm{J} 22533875+6242433$ & $22: 53: 38.74$ & $62: 42: 43.40$ & $-4.59 \pm 0.83$ & 12.78 \\
\hline $\mathrm{J} 22540370+6242122$ & $22: 54: 03.68$ & $62: 42: 12.35$ & $-19.16 \pm 9.52$ & 1.01 \\
\hline $\mathrm{J} 22540822+6243038$ & $22: 54: 08.23$ & $62: 43: 03.82$ & $-42.74 \pm 2.49$ & 3.70 \\
\hline $\mathrm{J} 22541822+6245397$ & $22: 54: 18.23$ & $62: 45: 39.83$ & $-117.54 \pm 0.99$ & 10.04 \\
\hline
\end{tabular}

\subsection{The RV Analysis Sample}

To reliably measure the velocity structure and dispersion of the Cep OB3b subclusters, we make three cuts to the RV data to ensure the reliability of the velocities. The first cut eliminates potential binaries as indicated in Section 3.1 (see Table 7). This cut reduces the sample from 190 to 109 . The second cut utilizes the $R$ value from rvsao, which is a measure of the signal-tonoise ratio. We reject objects that have an $R<5$ (Figure 2). The value adopted for this cut comes from an analysis of the threshold $R$ value versus the cumulative velocity dispersion, which examined the trade-off between sample sizes and the uncertainties in the RVs. In this analysis, the velocity dispersion was calculated for all the non-binary members at or below the $R$ value of the bin (Figure 2). We adopted the threshold for $R$, which gave the minimum velocity dispersion for a sample size exceeding 30 objects; this minimum occurred at $R \sim 5$. Increasing the threshold reduces the number of objects, leading to fluctuations from small numbers statistics. A lower threshold includes objects with poorly constrained RV values with inherently large uncertainties associated. This cut reduces the sample from 109 to 62 . The third cut is $3 \sigma$ clipping from the average velocity of the 62 remaining members in the cluster to reduce the contamination by nonmembers. The cut leaves 57 objects remaining.

\section{Results}

In the following section, we focus on the properties of the two subclusters. The rationale for this approach is based on two observations. First, while the overall cluster is highly elongated and hierarchical in structure, each of the two subclusters has a single, centrally condensed core, and is much closer to circular symmetry (Figure 4 in Allen et al. 2012). This suggests that these are two distinct components within the larger cluster that

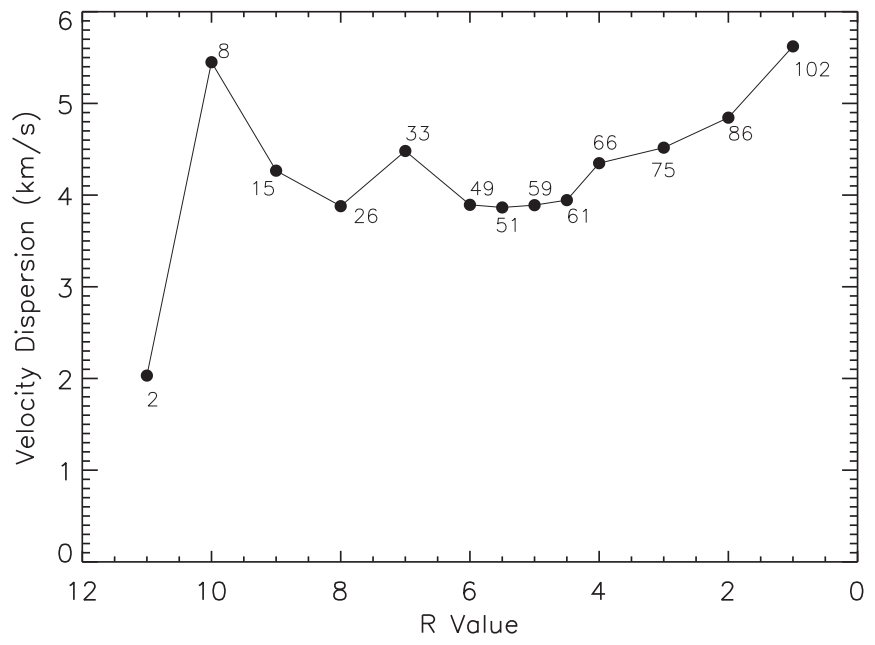

Figure 2. The velocity dispersion vs. minimum $R$ value of the stars with youth indicators, which are not identified as binaries. The number of objects included in the calculation is next to each $R$ value point. The velocity dispersion has a broad minimum around $R \sim 5$, which is adopted as the $R$ cutoff in Section 3.2.

can be studied independently. Furthermore, each subcluster is associated with a distinct molecular clump within the larger parental clouds (Figure 4 in Allen et al. 2012). After using RVs to assess membership, we first characterize the structure of the two subclusters, fitting them to the empirical King (1962) model to constrain their three-dimensional structure. We then analyze the RV structure and velocity dispersion of each subcluster. Finally, we use Gaia DR2 to determine the distance of Cep OB3b and the bulk proper motions of the subclusters. From these results, we can also estimate the gravitational potential energy of the subclusters. These properties will 
Table 5

2011 RV Results

\begin{tabular}{|c|c|c|c|c|}
\hline 2MASS ID & R.A. (J2000) & Decl. (J2000) & $\mathrm{RV} \pm \sigma\left(\mathrm{km} \mathrm{s}^{-1}\right)$ & $R$ Value \\
\hline $\mathrm{J} 22544381+6243128$ & $22: 54: 43.83$ & $62: 43: 12.75$ & $-31.44 \pm 2.01$ & 4.81 \\
\hline $\mathrm{J} 22535448+6244536$ & $22: 53: 54.47$ & $62: 44: 53.72$ & $44.65 \pm 4.18$ & 1.05 \\
\hline $\mathrm{J} 22542553+6244554$ & $22: 54: 25.56$ & $62: 44: 55.54$ & $-39.75 \pm 1.16$ & 8.70 \\
\hline $\mathrm{J} 22543560+6246312$ & $22: 54: 35.59$ & $62: 46: 31.16$ & $9.57 \pm 4.79$ & 2.31 \\
\hline $\mathrm{J} 22545800+6243449$ & $22: 54: 58.03$ & $62: 43: 44.95$ & $-30.83 \pm 1.43$ & 6.59 \\
\hline $\mathrm{J} 22544253+6245173$ & $22: 54: 42.52$ & $62: 45: 17.37$ & $-75.62 \pm 0.85$ & 11.72 \\
\hline $\mathrm{J} 22541822+6245397$ & $22: 54: 18.24$ & $62: 45: 39.83$ & $-121.27 \pm 0.70$ & 14.38 \\
\hline $\mathrm{J} 22543123+6245142$ & $22: 54: 31.25$ & $62: 45: 14.10$ & $-57.97 \pm 0.68$ & 14.66 \\
\hline $\mathrm{J} 22542135+6248095$ & $22: 54: 21.35$ & $62: 48: 09.36$ & $-9.31 \pm 1.12$ & 9.15 \\
\hline $\mathrm{J} 22545277+6246367$ & $22: 54: 52.76$ & $62: 46: 36.93$ & $-23.24 \pm 6.97$ & 1.85 \\
\hline $\mathrm{J} 22550561+6244055$ & 22:55:05.59 & 62:44:05.52 & $-27.65 \pm 13.85$ & 1.67 \\
\hline $\mathrm{J} 22542604+6255213$ & $22: 54: 26.06$ & $62: 55: 21.22$ & $-13.34 \pm 1.19$ & 8.31 \\
\hline $\mathrm{J} 22543806+6251022$ & $22: 54: 38.07$ & $62: 51: 02.27$ & $-37.44 \pm 0.74$ & 13.86 \\
\hline $\mathrm{J} 22543128+6251104$ & $22: 54: 31.28$ & $62: 51: 10.32$ & $-1.78 \pm 3.39$ & 4.65 \\
\hline $\mathrm{J} 22542299+6251039$ & $22: 54: 23.02$ & $62: 51: 03.80$ & $-18.08 \pm 18.35$ & 2.31 \\
\hline $\mathrm{J} 22541426+6254031$ & $22: 54: 14.30$ & $62: 54: 03.14$ & $-39.64 \pm 1.52$ & 8.38 \\
\hline $\mathrm{J} 22543667+6249120$ & $22: 54: 36.69$ & 62:49:11.94 & $-59.90 \pm 1.15$ & 10.34 \\
\hline $\mathrm{J} 22545297+6257070$ & $22: 54: 52.97$ & $62: 57: 06.98$ & $-30.78 \pm 0.59$ & 17.47 \\
\hline $\mathrm{J} 22544726+6249150$ & $22: 54: 47.28$ & $62: 49: 15.14$ & $-3.62 \pm 9.37$ & 2.03 \\
\hline $\mathrm{J} 22550150+6245130$ & $22: 55: 01.50$ & $62: 45: 13.20$ & $-61.67 \pm 1.09$ & 9.47 \\
\hline J22544094+6254169 & $22: 54: 40.96$ & $62: 54: 16.86$ & $-104.43 \pm 0.49$ & 21.14 \\
\hline $\mathrm{J} 22545426+6254405$ & $22: 54: 54.24$ & $62: 54: 40.48$ & $-105.19 \pm 0.54$ & 18.95 \\
\hline $\mathrm{J} 22550804+6244252$ & 22:55:08.05 & $62: 44: 25.41$ & $-5.57 \pm 1.83$ & 8.61 \\
\hline $\mathrm{J} 22551648+6250278$ & $22: 55: 16.48$ & $62: 50: 27.79$ & $-38.49 \pm 0.63$ & 16.58 \\
\hline $\mathrm{J} 22551475+6256151$ & $22: 55: 14.77$ & $62: 56: 15.06$ & $-83.59 \pm 0.70$ & 14.67 \\
\hline $\mathrm{J} 22550270+6249561$ & $22: 55: 02.73$ & $62: 49: 56.18$ & $-24.35 \pm 0.96$ & 11.37 \\
\hline $\mathrm{J} 22550699+6248278$ & 22:55:06.99 & $62: 48: 27.96$ & $-4.54 \pm 1.25$ & 9.25 \\
\hline $\mathrm{J} 22550543+6256090$ & $22: 55: 05.43$ & 62:56:08.90 & $-8.85 \pm 1.40$ & 7.48 \\
\hline $\mathrm{J} 22551246+6251268$ & $22: 55: 12.50$ & $62: 51: 26.65$ & $-21.77 \pm 1.96$ & 4.96 \\
\hline $\mathrm{J} 22552456+6255516$ & $22: 55: 24.59$ & $62: 55: 51.57$ & $-77.58 \pm 0.99$ & 10.74 \\
\hline $\mathrm{J} 22552154+6252561$ & $22: 55: 21.51$ & $62: 52: 56.16$ & $-23.65 \pm 5.80$ & 0.96 \\
\hline J22552191+6251339 & $22: 55: 21.17$ & $62: 51: 26.51$ & $-21.39 \pm 1.25$ & 8.41 \\
\hline $\mathrm{J} 22551559+6244263$ & $22: 55: 15.59$ & $62: 44: 26.27$ & $-28.56 \pm 0.67$ & 15.85 \\
\hline $\mathrm{J} 22552235+6250209$ & $22: 55: 22.32$ & $62: 50: 20.49$ & $-2.14 \pm 0.81$ & 11.45 \\
\hline $\mathrm{J} 22551829+6246369$ & $22: 55: 18.30$ & $62: 46: 37.00$ & $-7.95 \pm 0.91$ & 11.82 \\
\hline $\mathrm{J} 22552966+6251511$ & $22: 55: 29.68$ & $62: 51: 51.10$ & $-39.32 \pm 1.07$ & 9.40 \\
\hline $\mathrm{J} 22563443+6256189$ & $22: 56: 34.44$ & 62:56:18.94 & $-29.57 \pm 0.59$ & 17.62 \\
\hline $\mathrm{J} 22551760+6243231$ & $22: 55: 17.62$ & 62:43:22.99 & $-10.32 \pm 2.57$ & 7.24 \\
\hline $\mathrm{J} 22553931+6251006$ & $22: 55: 39.32$ & $62: 51: 00.64$ & $-6.83 \pm 1.54$ & 6.74 \\
\hline $\mathrm{J} 22553348+6246241$ & $22: 55: 31.82$ & $62: 46: 43.33$ & $-22.02 \pm 0.85$ & 12.70 \\
\hline $\mathrm{J} 22554785+6252037$ & $22: 55: 47.84$ & $62: 52: 03.65$ & $-13.88 \pm 7.14$ & 3.32 \\
\hline $\mathrm{J} 22554849+6250298$ & $22: 55: 48.50$ & $62: 50: 29.81$ & $-79.84 \pm 1.27$ & 7.51 \\
\hline $\mathrm{J} 22563239+6250214$ & $22: 56: 32.40$ & $62: 50: 21.45$ & $-37.40 \pm 2.79$ & 4.75 \\
\hline $\mathrm{J} 22561969+6253030$ & $22: 56: 19.70$ & $62: 53: 03.15$ & $0.47 \pm 2.55$ & 3.60 \\
\hline $\mathrm{J} 22560500+6248269$ & 22:56:05.02 & $62: 48: 26.79$ & $-17.79 \pm 1.99$ & 5.57 \\
\hline $\mathrm{J} 22560167+6250058$ & $22: 56: 01.66$ & $62: 50: 05.83$ & $-5.19 \pm 1.75$ & 5.26 \\
\hline $\mathrm{J} 22561016+6249328$ & $22: 56: 10.19$ & $62: 49: 32.87$ & $-24.22 \pm 6.05$ & 1.94 \\
\hline $\mathrm{J} 22564968+6255018$ & $22: 56: 49.68$ & 62:55:01.80 & $-35.14 \pm 2.19$ & 4.49 \\
\hline $\mathrm{J} 22560998+6250140$ & 22:56:09.99 & 62:50:13.96 & $-26.62 \pm 6.34$ & 1.51 \\
\hline $\mathrm{J} 22565588+6255095$ & $22: 56: 55.90$ & $62: 55: 09.67$ & $-19.10 \pm 3.93$ & 1.47 \\
\hline $\mathrm{J} 22555192+6244588$ & $22: 55: 51.94$ & $62: 44: 58.79$ & $-75.56 \pm 0.75$ & 14.27 \\
\hline $\mathrm{J} 22570096+6252271$ & $22: 57: 00.95$ & $62: 52: 27.09$ & $-29.46 \pm 0.66$ & 15.64 \\
\hline $\mathrm{J} 22565611+6249007$ & $22: 56: 56.14$ & 62:49:00.52 & $-5.76 \pm 0.99$ & 9.95 \\
\hline $\mathrm{J} 22553580+6245095$ & $22: 55: 35.85$ & $62: 45: 09.50$ & $-22.20 \pm 13.47$ & 1.01 \\
\hline $\mathrm{J} 22555107+6245182$ & $22: 55: 50.23$ & $62: 45: 24.68$ & $-28.66 \pm 6.66$ & 1.68 \\
\hline $\mathrm{J} 22561978+6248022$ & $22: 56: 19.81$ & $62: 48: 02.17$ & $-13.84 \pm 2.83$ & 3.99 \\
\hline $\mathrm{J} 22560985+6243379$ & $22: 56: 09.88$ & $62: 43: 38.11$ & $-31.21 \pm 0.92$ & 11.37 \\
\hline $\mathrm{J} 22563371+6246468$ & $22: 56: 33.73$ & $62: 46: 46.69$ & $-2.15 \pm 3.31$ & 2.95 \\
\hline $\mathrm{J} 22552722+6243290$ & $22: 55: 27.23$ & $62: 43: 28.92$ & $-11.74 \pm 4.74$ & 6.29 \\
\hline $\mathrm{J} 22560030+6245029$ & $22: 56: 01.27$ & $62: 44: 56.88$ & $-2.07 \pm 1.40$ & 7.30 \\
\hline $\mathrm{J} 22562963+6245284$ & $22: 56: 29.64$ & $62: 45: 28.52$ & $-9.57 \pm 2.37$ & 6.11 \\
\hline J22571517+6245499 & $22: 57: 15.19$ & $62: 45: 50.06$ & $-19.88 \pm 5.44$ & 1.86 \\
\hline $\mathrm{J} 22563906+6245092$ & $22: 56: 39.04$ & $62: 45: 09.22$ & $20.64 \pm 2.42$ & 4.14 \\
\hline $\mathrm{J} 22571391+6241355$ & $22: 57: 13.89$ & $62: 41: 35.44$ & $50.91 \pm 5.73$ & 1.89 \\
\hline
\end{tabular}


Table 5

(Continued)

\begin{tabular}{|c|c|c|c|c|}
\hline 2MASS ID & R.A. (J2000) & Decl. (J2000) & $\mathrm{RV} \pm \sigma\left(\mathrm{km} \mathrm{s}^{-1}\right)$ & $R$ Value \\
\hline $\mathrm{J} 22564067+6242109$ & $22: 56: 40.65$ & $62: 42: 10.91$ & $-66.88 \pm 0.77$ & 13.73 \\
\hline $\mathrm{J} 22562134+6243257$ & $22: 56: 21.34$ & $62: 43: 25.71$ & $-12.90 \pm 5.30$ & 4.50 \\
\hline $\mathrm{J} 22565123+6243197$ & $22: 56: 51.24$ & $62: 43: 19.74$ & $-11.04 \pm 3.76$ & 4.98 \\
\hline $\mathrm{J} 22561535+6242268$ & $22: 56: 15.34$ & $62: 42: 27.04$ & $-12.38 \pm 1.17$ & 9.29 \\
\hline $\mathrm{J} 22571812+6237011$ & $22: 57: 18.14$ & $62: 37: 00.98$ & $-25.59 \pm 1.05$ & 9.84 \\
\hline $\mathrm{J} 22562657+6241574$ & $22: 56: 26.58$ & $62: 41: 57.41$ & $-45.03 \pm 2.07$ & 4.98 \\
\hline $\mathrm{J} 22562637+6241289$ & $22: 56: 26.38$ & $62: 41: 28.94$ & $-13.97 \pm 1.40$ & 8.31 \\
\hline $\mathrm{J} 22570594+6238180$ & $22: 57: 05.96$ & $62: 38: 18.05$ & $-15.67 \pm 1.92$ & 6.00 \\
\hline $\mathrm{J} 22563654+6240077$ & $22: 56: 36.55$ & $62: 40: 07.52$ & $50.66 \pm 2.77$ & 3.65 \\
\hline $\mathrm{J} 22554285+6242312$ & $22: 55: 42.86$ & $62: 42: 30.97$ & $-33.58 \pm 0.86$ & 11.56 \\
\hline $\mathrm{J} 22553311+6240593$ & $22: 55: 33.10$ & $62: 40: 59.21$ & $-22.40 \pm 4.18$ & 1.16 \\
\hline $\mathrm{J} 22562974+6238533$ & $22: 56: 29.74$ & $62: 38: 53.37$ & $-10.48 \pm 1.28$ & 8.32 \\
\hline $\mathrm{J} 22555016+6240070$ & $22: 55: 50.18$ & $62: 40: 07.26$ & $-10.35 \pm 1.11$ & 10.8 \\
\hline $\mathrm{J} 22564271+6237475$ & $22: 56: 42.73$ & $62: 37: 47.51$ & $-28.33 \pm 5.58$ & 1.80 \\
\hline $\mathrm{J} 22563167+6237526$ & $22: 56: 31.70$ & $62: 37: 52.54$ & $-18.39 \pm 1.75$ & 7.46 \\
\hline $\mathrm{J} 22565692+6236389$ & $22: 56: 56.91$ & $62: 36: 38.80$ & $-14.70 \pm 10.37$ & 1.35 \\
\hline $\mathrm{J} 22560969+6237289$ & $22: 56: 09.69$ & $62: 37: 29.05$ & $-35.18 \pm 5.88$ & 1.52 \\
\hline $\mathrm{J} 22570845+6225441$ & $22: 57: 08.44$ & $62: 25: 44.29$ & $-83.85 \pm 0.65$ & 15.82 \\
\hline $\mathrm{J} 22570709+6230189$ & $22: 57: 07.08$ & $62: 30: 19.05$ & $-17.24 \pm 4.77$ & 2.95 \\
\hline $\mathrm{J} 22562274+6232501$ & $22: 56: 22.77$ & $62: 32: 50.12$ & $-13.19 \pm 5.34$ & 1.57 \\
\hline $\mathrm{J} 22561318+6235331$ & $22: 56: 13.19$ & $62: 35: 33.16$ & $3.34 \pm 2.50$ & 4.10 \\
\hline $\mathrm{J} 22555010+6237443$ & $22: 55: 50.12$ & $62: 37: 44.52$ & $-5.19 \pm 4.05$ & 6.29 \\
\hline $\mathrm{J} 22563234+6232404$ & $22: 56: 32.37$ & $62: 32: 40.44$ & $3.65 \pm 1.82$ & 5.07 \\
\hline $\mathrm{J} 22571116+6227330$ & $22: 57: 11.15$ & $62: 27: 33.00$ & $\cdots \pm \cdots$ & $\ldots$ \\
\hline $\mathrm{J} 22570603+6225208$ & $22: 57: 06.05$ & $62: 25: 20.84$ & $-33.78 \pm 1.25$ & 8.19 \\
\hline $\mathrm{J} 22554655+6233432$ & $22: 55: 46.59$ & $62: 33: 43.24$ & $-46.02 \pm 0.80$ & 13.00 \\
\hline $\mathrm{J} 22565217+6226391$ & $22: 56: 52.18$ & $62: 26: 39.07$ & $3.96 \pm 4.36$ & 1.36 \\
\hline $\mathrm{J} 22560135+6231397$ & $22: 56: 01.36$ & $62: 31: 39.52$ & $-52.00 \pm 0.74$ & 14.16 \\
\hline $\mathrm{J} 22552759+6241028$ & $22: 55: 27.58$ & $62: 41: 02.70$ & $-40.12 \pm 0.94$ & 10.77 \\
\hline $\mathrm{J} 22555686+6234332$ & $22: 55: 56.85$ & $62: 34: 33.24$ & $20.33 \pm 3.63$ & 3.05 \\
\hline $\mathrm{J} 22553701+6237109$ & $22: 55: 37.12$ & $62: 37: 17.38$ & $-36.14 \pm 0.92$ & 11.52 \\
\hline $\mathrm{J} 22553503+6235312$ & $22: 55: 35.02$ & $62: 35: 31.05$ & $-104.19 \pm 0.85$ & 12.46 \\
\hline $\mathrm{J} 22551738+6241070$ & $22: 55: 17.40$ & $62: 41: 06.94$ & $-12.08 \pm 5.19$ & 0.69 \\
\hline $\mathrm{J} 22552505+6236511$ & $22: 55: 25.04$ & $62: 36: 51.19$ & $-11.53 \pm 1.46$ & 7.51 \\
\hline $\mathrm{J} 22553247+6231367$ & $22: 55: 32.43$ & $62: 31: 36.71$ & $-20.45 \pm 6.31$ & 2.13 \\
\hline $\mathrm{J} 22555006+6223083$ & $22: 55: 50.06$ & $62: 23: 08.26$ & $-14.02 \pm 4.15$ & 4.34 \\
\hline $\mathrm{J} 22545509+6233404$ & $22: 54: 55.13$ & $62: 33: 40.13$ & $-29.54 \pm 1.05$ & 9.85 \\
\hline $\mathrm{J} 22550059+6235221$ & $22: 55: 00.60$ & $62: 35: 22.06$ & $-8.63 \pm 1.00$ & 10.82 \\
\hline $\mathrm{J} 22551657+6232522$ & $22: 55: 16.59$ & $62: 32: 52.13$ & $-28.72 \pm 4.32$ & 1.85 \\
\hline $\mathrm{J} 22550590+6234504$ & 22:55:05.91 & $62: 34: 50.53$ & $-10.10 \pm 2.77$ & 6.97 \\
\hline $\mathrm{J} 22550975+6239074$ & $22: 55: 09.71$ & $62: 39: 07.24$ & $29.33 \pm 6.39$ & 3.09 \\
\hline $\mathrm{J} 22543882+6223364$ & $22: 54: 38.83$ & $62: 23: 36.62$ & $-11.17 \pm 0.83$ & 12.57 \\
\hline $\mathrm{J} 22543713+6226052$ & $22: 54: 37.18$ & $62: 26: 05.50$ & $-5.87 \pm 0.97$ & 10.22 \\
\hline $\mathrm{J} 22540555+6223064$ & $22: 54: 05.54$ & $62: 23: 06.65$ & $-28.15 \pm 0.98$ & 10.87 \\
\hline $\mathrm{J} 22545903+6237177$ & $22: 54: 59.04$ & $62: 37: 17.82$ & $0.88 \pm 10.67$ & 3.83 \\
\hline $\mathrm{J} 22542760+6225270$ & $22: 54: 27.60$ & $62: 25: 26.88$ & $-5.50 \pm 2.60$ & 4.56 \\
\hline $\mathrm{J} 22541824+6226460$ & $22: 54: 18.24$ & $62: 26: 45.93$ & $5.93 \pm 8.75$ & 2.48 \\
\hline $\mathrm{J} 22542257+6225041$ & $22: 54: 22.56$ & $62: 25: 04.03$ & $-13.77 \pm 1.77$ & 7.82 \\
\hline $\mathrm{J} 22531916+6225326$ & $22: 53: 19.20$ & $62: 25: 32.83$ & $17.33 \pm 1.17$ & 9.06 \\
\hline $\mathrm{J} 22542405+6235158$ & $22: 54: 24.05$ & $62: 35: 15.95$ & $-14.11 \pm 2.62$ & 5.81 \\
\hline $\mathrm{J} 22544431+6235183$ & $22: 54: 44.32$ & $62: 35: 18.23$ & $12.42 \pm 0.92$ & 11.68 \\
\hline $\mathrm{J} 22565314+6232094$ & $22: 54: 33.12$ & $62: 33: 33.84$ & $4.54 \pm 1.45$ & 7.23 \\
\hline $\mathrm{J} 22541607+6230398$ & $22: 54: 16.08$ & $62: 30: 39.76$ & $-19.88 \pm 1.02$ & 10.33 \\
\hline $\mathrm{J} 22543423+6235555$ & $22: 54: 34.20$ & $62: 35: 55.46$ & $-4.98 \pm 6.67$ & 3.47 \\
\hline $\mathrm{J} 22534870+6236019$ & $22: 53: 48.70$ & $62: 36: 01.81$ & $-11.92 \pm 1.38$ & 8.27 \\
\hline $\mathrm{J} 22544294+6238557$ & $22: 54: 42.93$ & $62: 38: 55.74$ & $-1.53 \pm 2.27$ & 5.51 \\
\hline $\mathrm{J} 22534931+6235274$ & $22: 53: 49.29$ & $62: 35: 27.52$ & $-9.69 \pm 1.53$ & 7.85 \\
\hline $\mathrm{J} 22533629+6231446$ & $22: 53: 36.28$ & $62: 31: 44.69$ & $-12.69 \pm 1.58$ & 8.31 \\
\hline $\mathrm{J} 22523242+6227422$ & $22: 52: 32.41$ & $62: 27: 42.40$ & $-3.32 \pm 15.06$ & 1.18 \\
\hline $\mathrm{J} 22544332+6240103$ & $22: 54: 43.31$ & $62: 40: 10.26$ & $-10.47 \pm 1.85$ & 6.37 \\
\hline $\mathrm{J} 22540553+6235451$ & $22: 54: 05.56$ & $62: 35: 45.03$ & $-10.99 \pm 4.59$ & 1.94 \\
\hline $\mathrm{J} 22523057+6226259$ & $22: 52: 30.58$ & $62: 26: 25.86$ & $8.59 \pm \cdots$ & $\cdots$ \\
\hline $\mathrm{J} 22530352+6237081$ & $22: 53: 03.52$ & $62: 37: 08.00$ & $-16.42 \pm 8.63$ & 0.73 \\
\hline $\mathrm{J} 22540731+6238098$ & $22: 54: 06.84$ & $62: 38: 06.57$ & $-45.49 \pm 1.32$ & 7.63 \\
\hline
\end{tabular}


Table 5

(Continued)

\begin{tabular}{|c|c|c|c|c|}
\hline 2MASS ID & R.A. (J2000) & Decl. (J2000) & $\mathrm{RV} \pm \sigma\left(\mathrm{km} \mathrm{s}^{-1}\right)$ & $R$ Value \\
\hline $\mathrm{J} 22530628+6236326$ & $22: 53: 06.30$ & $62: 36: 32.69$ & $-13.22 \pm 6.26$ & 0.65 \\
\hline $\mathrm{J} 22540271+6238389$ & $22: 54: 02.70$ & $62: 38: 39.06$ & $-27.36 \pm 0.93$ & 10.69 \\
\hline $\mathrm{J} 22534450+6238305$ & $22: 53: 44.50$ & $62: 38: 30.41$ & $-25.15 \pm 16.73$ & 0.78 \\
\hline $\mathrm{J} 22523176+6231194$ & $22: 52: 31.80$ & $62: 31: 19.69$ & $-2.13 \pm 1.42$ & 6.79 \\
\hline $\mathrm{J} 22524589+6234125$ & $22: 52: 45.92$ & $62: 34: 12.53$ & $57.21 \pm 3.88$ & 1.31 \\
\hline $\mathrm{J} 22533602+6236494$ & $22: 53: 36.02$ & $62: 36: 49.34$ & $-20.27 \pm 7.09$ & 1.46 \\
\hline $\mathrm{J} 22524939+6233534$ & $22: 52: 49.40$ & $62: 33: 53.57$ & $-30.23 \pm 1.69$ & 5.82 \\
\hline $\mathrm{J} 22534448+6239061$ & $22: 53: 44.44$ & $62: 39: 06.52$ & $-19.65 \pm 2.62$ & 4.09 \\
\hline $\mathrm{J} 22524772+6240044$ & $22: 52: 47.71$ & $62: 40: 04.72$ & $1.69 \pm 8.11$ & 1.58 \\
\hline $\mathrm{J} 22530063+6237581$ & $22: 53: 00.63$ & $62: 37: 58.14$ & $30.89 \pm 2.00$ & 5.61 \\
\hline $\mathrm{J} 23033097+6239264$ & $22.52: 32.32$ & $62: 39: 25.12$ & $-31.47 \pm 1.76$ & 5.22 \\
\hline $\mathrm{J} 22531297+6238444$ & $22: 53: 13.00$ & $62: 38: 44.41$ & $30.05 \pm 1.47$ & 7.91 \\
\hline $\mathrm{J} 22543623+6241336$ & $22: 54: 36.24$ & $62: 41: 33.81$ & $-39.31 \pm 2.33$ & 4.24 \\
\hline $\mathrm{J} 22530993+6239119$ & $22: 53: 09.92$ & $62: 39: 12.17$ & $0.17 \pm 2.68$ & 3.41 \\
\hline $\mathrm{J} 22523061+6238279$ & $22: 52: 30.61$ & $62: 38: 28.16$ & $-19.52 \pm 3.09$ & 2.98 \\
\hline $\mathrm{J} 22533857+6244222$ & $22: 53: 38.60$ & $62: 44: 22.21$ & $-18.58 \pm 1.82$ & 5.49 \\
\hline $\mathrm{J} 22525023+6240458$ & $22: 52: 50.26$ & $62: 40: 45.98$ & $-49.40 \pm 1.38$ & 7.40 \\
\hline $\mathrm{J} 22535512+6243368$ & $22: 53: 55.12$ & $62: 43: 36.90$ & $-14.02 \pm 4.03$ & 1.27 \\
\hline $\mathrm{J} 22522749+6241104$ & $22: 52: 27.48$ & $62: 41: 10.30$ & $15.96 \pm 5.39$ & 0.79 \\
\hline $\mathrm{J} 22523381+6243331$ & $22: 52: 32.67$ & $62: 43: 37.18$ & $-22.10 \pm 2.36$ & 3.74 \\
\hline $\mathrm{J} 22541786+6242328$ & $22: 54: 17.85$ & $62: 42: 32.91$ & $-107.30 \pm 1.02$ & 9.91 \\
\hline $\mathrm{J} 22535737+6243018$ & $22: 53: 57.38$ & $62: 43: 01.84$ & $-61.13 \pm 0.88$ & 11.35 \\
\hline $\mathrm{J} 22522631+6242084$ & $22: 52: 26.33$ & $62: 42: 08.72$ & $0.48 \pm 0.78$ & 12.95 \\
\hline $\mathrm{J} 22531323+6242295$ & $22: 53: 13.27$ & $62: 42: 29.46$ & $-36.62 \pm 4.85$ & 1.47 \\
\hline
\end{tabular}

inform a discussion of the dynamical status and fate of the subclusters in subsequent sections.

\subsection{RV Analysis of Membership}

Due to the low extinction through the cluster, Hectoschelle can detect both foreground and background objects in the field in addition to members of the cluster. We therefore break up the sample into two categories: objects with youth indicators and objects without youth indicators, most of which are likely contaminants. Youth indicators are the presence of an infrared excess, implying the presence of a dusty disk or envelope (i.e., class II objects, transition-disk objects, or protostars), or detectable X-ray emission due to an active corona. The objects with X-ray emission but no IR excess are diskless pre-mainsequence (pre-MS) stars (class III objects). The velocities of the stars with youth indicators show a clear peak in the RV of $-10 \mathrm{~km} \mathrm{~s}^{-1}$ bin with a narrow RV distribution (Figure 3).

Objects without youth indicators may also be class III objects; however, such objects are indistinguishable from background giants or foreground dwarf objects on the basis of photometry alone. Figure 3 shows histograms for objects with youth indicators and objects without youth indicators. The objects with youth indicators show a clear peak at $-10 \mathrm{~km} \mathrm{~s}^{-1}$. In contrast, the histogram of the objects without youth indicators (169) shows a broad distribution of RVs ranging from -107 to $50 \mathrm{~km} \mathrm{~s}^{-1}$. There is a peak at $-30 \mathrm{~km} \mathrm{~s}^{-1}$, which is consistent with the RVs of stars in the Perseus arm behind Cep OB3b. At the velocity of the cluster, $-10 \mathrm{~km} \mathrm{~s}^{-1}$, the distribution of the objects without youth indicators is relatively flat compared to the youth indicators, consistent with objects dominated by field stars. Thus, we see no clear evidence for a large number of missed class III objects lacking X-ray detection in the RV distribution, and we do not use RV as an additional criterion for membership.
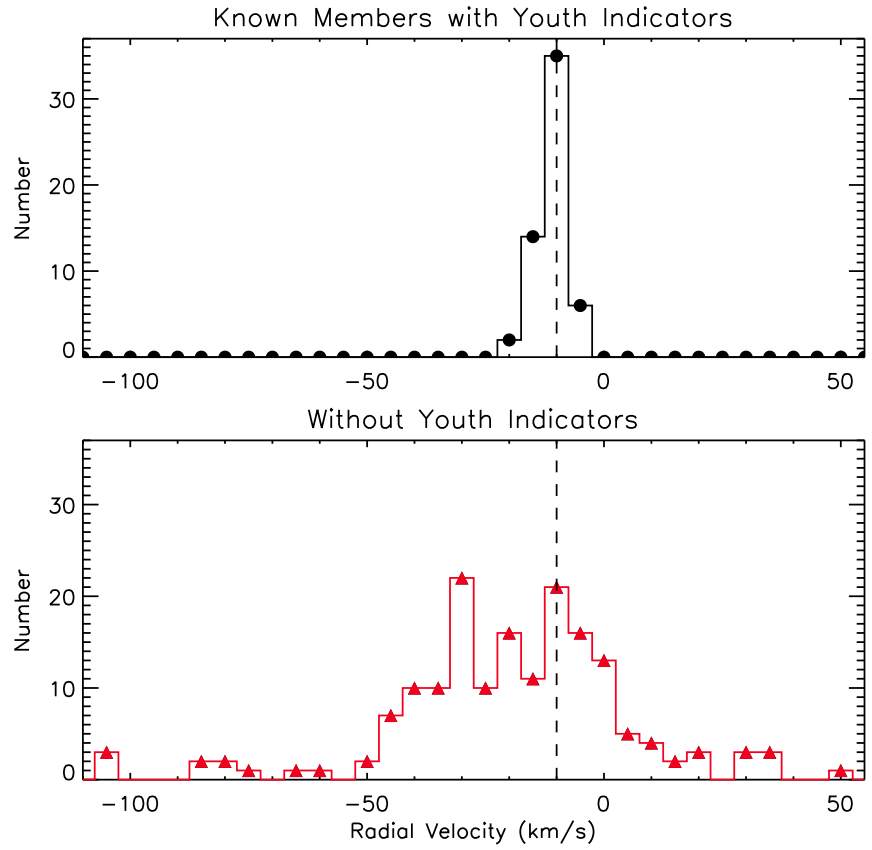

Figure 3. Radial velocity distribution of objects with youth indicators (top) and objects lacking youth indicators (bottom). The molecular cloud peaks at $-10 \mathrm{~km} \mathrm{~s}^{-1}$, which agrees with the LSR of the molecular cloud. More local clouds like Cep OB3b are found closer to $0 \mathrm{~km} \mathrm{~s}^{-1}$. Objects with highly negative RVs are likely background stars, with objects in the Perseus arm peaking at $-30 \mathrm{~km} \mathrm{~s}^{-1}$.

Heyer et al. (1996) imaged ${ }^{13} \mathrm{CO}$ and ${ }^{12} \mathrm{CO} \quad J=1-0$ emission from visible $\mathrm{H}$ II regions, which included Cep OB3b. The ${ }^{12} \mathrm{CO}$ gas toward the cluster was measured at -12.7 , 
Table 6

2013 RV Results

\begin{tabular}{|c|c|c|c|c|}
\hline 2MASS ID & R.A. (J2000) & Decl. (J2000) & $\overline{R V} \pm \sigma\left(\mathrm{km} \mathrm{s}^{-1}\right)$ & $\overline{R \text { Value }}$ \\
\hline $\mathrm{J} 22562637+6241289$ & $22: 56: 26.37$ & $62: 41: 28.97$ & $-10.59 \pm 1.63$ & 7.30 \\
\hline $\mathrm{J} 22560480+6239460$ & $22: 56: 04.80$ & $62: 39: 46.09$ & $-9.73 \pm 1.00$ & 9.98 \\
\hline $\mathrm{J} 22571825+6243042$ & $22: 57: 18.25$ & $62: 43: 04.27$ & $-11.13 \pm 2.17$ & 4.56 \\
\hline $\mathrm{J} 22562231+6240488$ & $22: 56: 22: 31$ & $62: 40: 48.81$ & $-9.85 \pm 1.35$ & 7.90 \\
\hline J22570134+6242133 & 22:57:01.35 & 62:42:13.39 & $11.91 \pm 4.25$ & 2.77 \\
\hline $\mathrm{J} 22570594+6238180$ & $22: 57: 05.95$ & $62: 38: 18.07$ & $-13.39 \pm 2.49$ & 3.43 \\
\hline $\mathrm{J} 22570917+6238560$ & $22: 57: 09.18$ & $62: 38: 56.06$ & $-16.38 \pm 4.78$ & 2.88 \\
\hline $\mathrm{J} 22565186+6239521$ & $22: 56: 51.86$ & $62: 39: 52.12$ & $-14.38 \pm 1.81$ & 6.48 \\
\hline $\mathrm{J} 22563527+6239074$ & $22: 56: 35.27$ & $62: 39: 07.45$ & $-7.01 \pm 1.30$ & 9.09 \\
\hline $\mathrm{J} 22564210+6236207$ & $22: 56: 42.10$ & $62: 36: 20.73$ & $-16.31 \pm 1.83$ & 5.28 \\
\hline $\mathrm{J} 22564271+6237475$ & $22: 56: 42.71$ & $62: 37: 47.52$ & $-11.78 \pm 3.74$ & 2.51 \\
\hline $\mathrm{J} 22554154+6239030$ & $22: 55: 41.54$ & $62: 39: 03.07$ & $19.25 \pm 4.88$ & 2.05 \\
\hline $\mathrm{J} 22564720+6237055$ & $22: 56: 47.20$ & $62: 37: 05.56$ & $-5.47 \pm 2.24$ & 7.81 \\
\hline $\mathrm{J} 22555010+6237443$ & $22: 55: 50.10$ & $62: 37: 44.32$ & $-9.30 \pm 2.45$ & 8.34 \\
\hline $\mathrm{J} 22561343+6236452$ & $22: 56: 13.43$ & $62: 36: 45.20$ & $-17.69 \pm 3.26$ & 3.04 \\
\hline $\mathrm{J} 22570919+6233337$ & $22: 57: 09.20$ & $62: 33: 33.75$ & $1.81 \pm 10.08$ & 3.20 \\
\hline $\mathrm{J} 22562852+6232301$ & $22: 56: 28.52$ & $62: 32: 30.11$ & $1.46 \pm 1.19$ & 9.03 \\
\hline $\mathrm{J} 22561318+6235331$ & $22: 56: 13.18$ & $62: 35: 33.15$ & $12.59 \pm 2.08$ & 4.65 \\
\hline $\mathrm{J} 22571251+6228058$ & $22: 57: 12.51$ & $62: 28: 05.84$ & $-51.42 \pm 0.69$ & 15.11 \\
\hline $\mathrm{J} 22555686+6234332$ & $22: 55: 56.86$ & $62: 34: 33.29$ & $22.35 \pm 1.49$ & 7.88 \\
\hline $\mathrm{J} 22561122+6233290$ & $22: 56: 11.22$ & $62: 33: 29.08$ & $-30.20 \pm 7.49$ & 1.96 \\
\hline $\mathrm{J} 22560790+6230425$ & 22:56:07.91 & $62: 30: 42.59$ & $-10.36 \pm 1.97$ & 5.29 \\
\hline $\mathrm{J} 22554370+6234569$ & $22: 55: 43.70$ & $62: 34: 56.97$ & $-14.83 \pm 0.55$ & 19.03 \\
\hline $\mathrm{J} 22553370+6233093$ & $22: 55: 33.70$ & 62:33:09.34 & $-25.55 \pm 1.44$ & 9.28 \\
\hline $\mathrm{J} 22553606+6238563$ & $22: 55: 36.25$ & $62: 39: 02.35$ & $-6.12 \pm 5.42$ & 4.23 \\
\hline $\mathrm{J} 22552505+6236511$ & $22: 55: 25.05$ & $62: 36: 51.16$ & $-11.19 \pm 1.45$ & 8.41 \\
\hline $\mathrm{J} 22550059+6235221$ & $22: 55: 00.60$ & $62: 35: 22.17$ & $-9.93 \pm 1.03$ & 9.67 \\
\hline $\mathrm{J} 22550590+6234504$ & $22: 55: 05.90$ & $62: 34: 50.47$ & $-23.59 \pm 2.57$ & 4.90 \\
\hline $\mathrm{J} 22545364+6234031$ & $22: 54: 53.64$ & $62: 34: 03.10$ & $-18.24 \pm 2.54$ & 6.32 \\
\hline $\mathrm{J} 22535527+6233218$ & $22: 53: 55.27$ & $62: 33: 21.85$ & $-16.36 \pm 8.71$ & 2.36 \\
\hline $\mathrm{J} 22544224+6235548$ & $22: 54: 42.24$ & $62: 35: 54.89$ & $-8.70 \pm 1.09$ & 9.57 \\
\hline $\mathrm{J} 22525012+6227336$ & $22: 52: 50.12$ & $62: 27: 33.64$ & $-3.87 \pm 7.60$ & 1.13 \\
\hline $\mathrm{J} 22552178+6237535$ & $22: 55: 21.78$ & $62: 37: 53.55$ & $-5.31 \pm 6.22$ & 1.59 \\
\hline $\mathrm{J} 22543423+6235555$ & $22: 54: 34.23$ & $62: 35: 55.55$ & $-17.14 \pm 6.54$ & 2.57 \\
\hline $\mathrm{J} 22531578+6235262$ & $22: 53: 15.78$ & $62: 35: 26.27$ & $-22.32 \pm 6.54$ & 1.17 \\
\hline $\mathrm{J} 22541270+6235379$ & $22: 54: 12.70$ & $62: 35: 37.95$ & $-23.91 \pm 6.71$ & 3.44 \\
\hline $\mathrm{J} 22532187+6234490$ & $22: 53: 21.87$ & $62: 34: 49.04$ & $-22.64 \pm 2.63$ & 3.28 \\
\hline $\mathrm{J} 22535132+6235184$ & $22: 53: 51.32$ & $62: 35: 18.43$ & $-9.67 \pm 1.21$ & 8.42 \\
\hline $\mathrm{J} 22523829+6232503$ & $22: 52: 38.29$ & $62: 32: 50.32$ & $12.30 \pm 1.01$ & 9.02 \\
\hline $\mathrm{J} 22532653+6240134$ & $22: 53: 26.53$ & $62: 40: 13.45$ & $-8.52 \pm 1.14$ & 9.33 \\
\hline $\mathrm{J} 22543166+6237117$ & $22: 54: 31.66$ & $62: 37: 11.69$ & $-6.16 \pm 1.83$ & 6.03 \\
\hline $\mathrm{J} 22540382+6238211$ & $22: 54: 03.82$ & $62: 38: 21.11$ & $-13.10 \pm 1.00$ & 10.6 \\
\hline $\mathrm{J} 22530421+6236112$ & $22: 53: 04.22$ & $62: 36: 11.26$ & $-22.52 \pm 6.14$ & 2.14 \\
\hline $\mathrm{J} 22530063+6237581$ & 22:53:00.64 & $62: 37: 58.19$ & $-22.68 \pm 0.96$ & 11.23 \\
\hline $\mathrm{J} 22531015+6236444$ & $22: 53: 10.15$ & $62: 36: 44.42$ & $-11.57 \pm 2.96$ & 4.68 \\
\hline $\mathrm{J} 22532283+6237409$ & $22: 53: 22.83$ & $62: 37: 40.97$ & $-9.94 \pm 2.21$ & 5.19 \\
\hline $\mathrm{J} 22533869+6237171$ & $22: 53: 38.69$ & $62: 37: 17.11$ & $-12.45 \pm 4.94$ & 4.20 \\
\hline $\mathrm{J} 22544942+6239227$ & $22: 54: 49.42$ & $62: 39: 22.78$ & $-12.36 \pm 1.03$ & 10.40 \\
\hline $\mathrm{J} 22531480+6244040$ & $22: 53: 14.80$ & $62: 44: 04.05$ & $-38.09 \pm 0.85$ & 12.05 \\
\hline $\mathrm{J} 22542993+6240335$ & $22: 54: 29.93$ & $62: 40: 33.55$ & $-7.88 \pm 1.67$ & 6.96 \\
\hline $\mathrm{J} 22530099+6243548$ & $22: 53: 01.00$ & $62: 43: 54.82$ & $1.69 \pm 4.33$ & 3.60 \\
\hline $\mathrm{J} 22555158+6239116$ & $22: 55: 51.58$ & $62: 39: 11.69$ & $-6.92 \pm 1.38$ & 7.69 \\
\hline $\mathrm{J} 22535512+6243368$ & $22: 53: 55.12$ & $62: 43: 36.88$ & $-20.39 \pm 17.09$ & 0.23 \\
\hline $\mathrm{J} 22551643+6240321$ & $22: 55: 16.43$ & $62: 40: 32.13$ & $-8.07 \pm 0.99$ & 10.30 \\
\hline $\mathrm{J} 22550275+6241499$ & $22: 55: 02.75$ & $62: 41: 49.96$ & $-15.14 \pm 3.54$ & 1.42 \\
\hline $\mathrm{J} 22550959+6244210$ & $22: 55: 09.60$ & $62: 44: 21.03$ & $-21.37 \pm 4.58$ & 5.19 \\
\hline $\mathrm{J} 22550561+6244055$ & 22:55:05.61 & 62:44:05.56 & $-26.24 \pm 7.41$ & 2.67 \\
\hline $\mathrm{J} 22544992+6251217$ & $22: 54: 49.92$ & $62: 51: 21.78$ & $-10.45 \pm 1.52$ & 9.46 \\
\hline $\mathrm{J} 22552722+6243290$ & $22: 55: 27.22$ & $62: 43: 29.03$ & $-14.88 \pm 4.14$ & 7.02 \\
\hline $\mathrm{J} 22551760+6243231$ & $22: 55: 17.60$ & $62: 43: 23.18$ & $-10.45 \pm 2.77$ & 6.94 \\
\hline $\mathrm{J} 22552486+6246446$ & $22: 55: 24.86$ & $62: 46: 44.63$ & $6.10 \pm 4.81$ & 2.88 \\
\hline $\mathrm{J} 22560150+6247467$ & $22: 56: 01.50$ & $62: 47: 46.79$ & $-10.70 \pm 1.57$ & 7.81 \\
\hline $\mathrm{J} 22555228+6249200$ & $22: 55: 52.28$ & $62: 49: 20.07$ & $-6.41 \pm 0.88$ & 10.9 \\
\hline $\mathrm{J} 22555152+6245053$ & $22: 55: 51.52$ & $62: 45: 05.40$ & $-9.88 \pm 1.32$ & 7.77 \\
\hline
\end{tabular}


Table 6

(Continued)

\begin{tabular}{lccc}
\hline \hline 2MASS ID & R.A. (J2000) & Decl. (J2000) & RV $\pm \sigma\left(\mathrm{km} \mathrm{s}^{-1}\right)$ \\
\hline J22561829+6245166 & $22: 56: 18.29$ & $62: 45: 16.62$ & $-17.19 \pm 4.97$ \\
J22560391+6243359 & $22: 56: 03.92$ & $62: 43: 35.90$ & $-11.24 \pm 1.13$ \\
J22563677+6255599 & $22: 56: 36.77$ & $62: 55: 59.94$ & $-16.43 \pm 4.40$ \\
J22554749+6241576 & $22: 55: 47.49$ & $62: 41: 57.61$ & $-13.00 \pm 2.43$ \\
J22560068+6245310 & $22: 56: 00.69$ & $62: 45: 31.02$ & $-9.43 \pm 1.37$ \\
J22562134+6243257 & $22: 56: 21.34$ & $62: 43: 25.72$ & $6.65 \pm 9.85$ \\
J22560428+6242388 & $22: 56: 04.28$ & $62: 42: 38.85$ & $-9.92 \pm 21.54$ \\
J22564512+6246193 & $22: 56: 45.12$ & $62: 46: 19.32$ & $-10.50 \pm 2.09$ \\
J22562963+6245284 & $22: 56: 29.63$ & $62: 45: 28.44$ & $-11.15 \pm 2.29$ \\
J22565123+6243197 & $22: 56: 51.23$ & $62: 43: 19.76$ & $-11.81 \pm 2.68$ \\
J22555016+6240070 & $22: 55: 50.16$ & $62: 40: 07.09$ & $-9.56 \pm 1.60$ \\
J22564619+6244225 & $22: 56: 46.19$ & $62: 44: 22.59$ & $-15.71 \pm 1.31$ \\
J22561535+6242268 & $22: 56: 15.35$ & $62: 42: 26.86$ & $-13.04 \pm 1.04$ \\
\hline
\end{tabular}

$-11.4,-10.1,-8.8$, and $-7.5 \mathrm{~km} \mathrm{~s}^{-1}$, which is consistent with the motions of the members. We do not see a significant offset between the stellar motions and the gas motions, further confirmation of their association with the cloud.

\subsection{Subcluster Sizes, Structures, and Densities}

To characterize the structures of the subclusters, we apply an analytic model developed by King (1962) for globular clusters. The models are parameterized by a core radius, an outer tidal radius, and a central peak density. Although this model is not appropriate for the elongated and irregular structure of more deeply embedded clusters such as the ONC (Kuhn et al. 2014; Megeath et al. 2016), the more circular symmetry of the two Cep OB3b subclusters motivates the use of this model. We use the Chandra and Spitzer data for the King model fits. We adopt the center positions of the subclusters given by the method of Gutermuth et al. (2009). The algorithm isolates the two most numerous YSO overdensities that lack further substructure using the catalog and field-of-view limits of Allen et al. (2012). We compute the azimuthally averaged radial surface density profile in $0.3 \mathrm{pc}$ bins and fit them with King models using the IDL implementation of mpfit, specifically the mpcurvefit.pro module (Markwardt 2009).

Since the larger annuli extended in part past the Chandra field of view, we must correct for the missing Chandra objects. We do this by measuring the density of the X-ray objects for the section of the annulus within the Chandra field of view. In addition, we must correct for the stars that did not have infrared excesses and did not have bright enough X-ray emissions to be detected by Chandra. Allen et al. (2012) determined disk fractions in each subcluster using visible color-magnitude diagrams and the X-ray data (see Table 5 Allen et al. 2012). We adopt the upper and lower values of the disk fractions for each subcluster to correct for missing objects. We do this by determining a correction factor for each subcluster, given by the equation

$$
\eta=\frac{1}{f_{\text {disk }}} \frac{N_{\mathrm{IR}}}{N_{\mathrm{IR}}+N_{\mathrm{X}-\text { ray }}},
$$

where $N_{\text {IR }}$ is the number of IR-excess objects with the boundary of the given subclusters, $N_{\mathrm{X} \text {-ray }}$ is the number of Chandra objects, and $f_{\text {disk }}$ is the disk fraction. This assumes that $N_{\text {IR }} / f_{\text {disk }}$ gives the total number of objects. Finally, we take the total number of stars from the Chandra and Spitzer data sets in each subcluster and subtract off a $15.5 \mathrm{pc}^{-2}$ baseline density. This was done to (1) exclude the larger scale halo of YSOs that are likely part of the larger Cep OB3b cluster and (2) to keep the two subclusters separate. After the $15.5 \mathrm{pc}^{-2}$ baseline density is subtracted, the measured densities are multiplied by the correction factor to determine the corrected density of stars in each annulus. This yields 809 and 664 stars for disk fractions of 0.32 and 0.39 , respectively, in the east and 501 and 416 stars for disk fractions of 0.44 and 0.53 , respectively, in the west. The number of objects determined here are used in the potential energy calculations.

The fits give different results for the two subclusters (Figure 4, Table 8). The core radii are $1.36 \pm 0.30 \mathrm{pc}$ and $0.52 \pm 0.11 \mathrm{pc}$ for the east and west, respectively. The tidal radii are $2.32 \pm 0.19 \mathrm{pc}$ and $3.1 \pm 1.0 \mathrm{pc}$ for the east and west, respectively. We note that the tidal radius usually refers to the radius at which the tidal field of the galaxy dominates over the gravity of the cluster, separating stars out of the cluster. Here, the tidal radius defines the edge of the subclusters in the King model, and we will refer to it as the subcluster radius throughout the remainder of the paper.

In the above analysis, we have assumed that the two subclusters are circularly symmetric. To test how closely the clusters follow this assumption, we compute the AAP from Gutermuth et al. (2005) for the two subclusters. The value of the AAP measures the deviation of the azimuthal distribution of stars from circular symmetry. We find values of 1.155 and 1.724 for the east and west, respectively. This shows that the surface density distribution of stars in the west deviate significantly from circular symmetry and that the subcluster is significantly elongated. In comparison, the east appears circularly symmetric with a relatively smooth distribution of stars.

The stellar peak densities are 521 and 428 stars pc $^{-2}$ in the east and 342 and 284 stars $\mathrm{pc}^{-2}$ in the west, depending on the disk fraction. The average stellar surface densities within the core radii are 240 and 197 stars pc $^{-2}$ in the east and 220 and 182 stars $\mathrm{pc}^{-2}$ in the west, depending on the disk fraction. From these values, the east is significantly richer in members than the west and is circularly symmetric, showing a larger core radius. The west is characterized by elongation, a core radius that is less than half of that for the east, and has fewer members. 

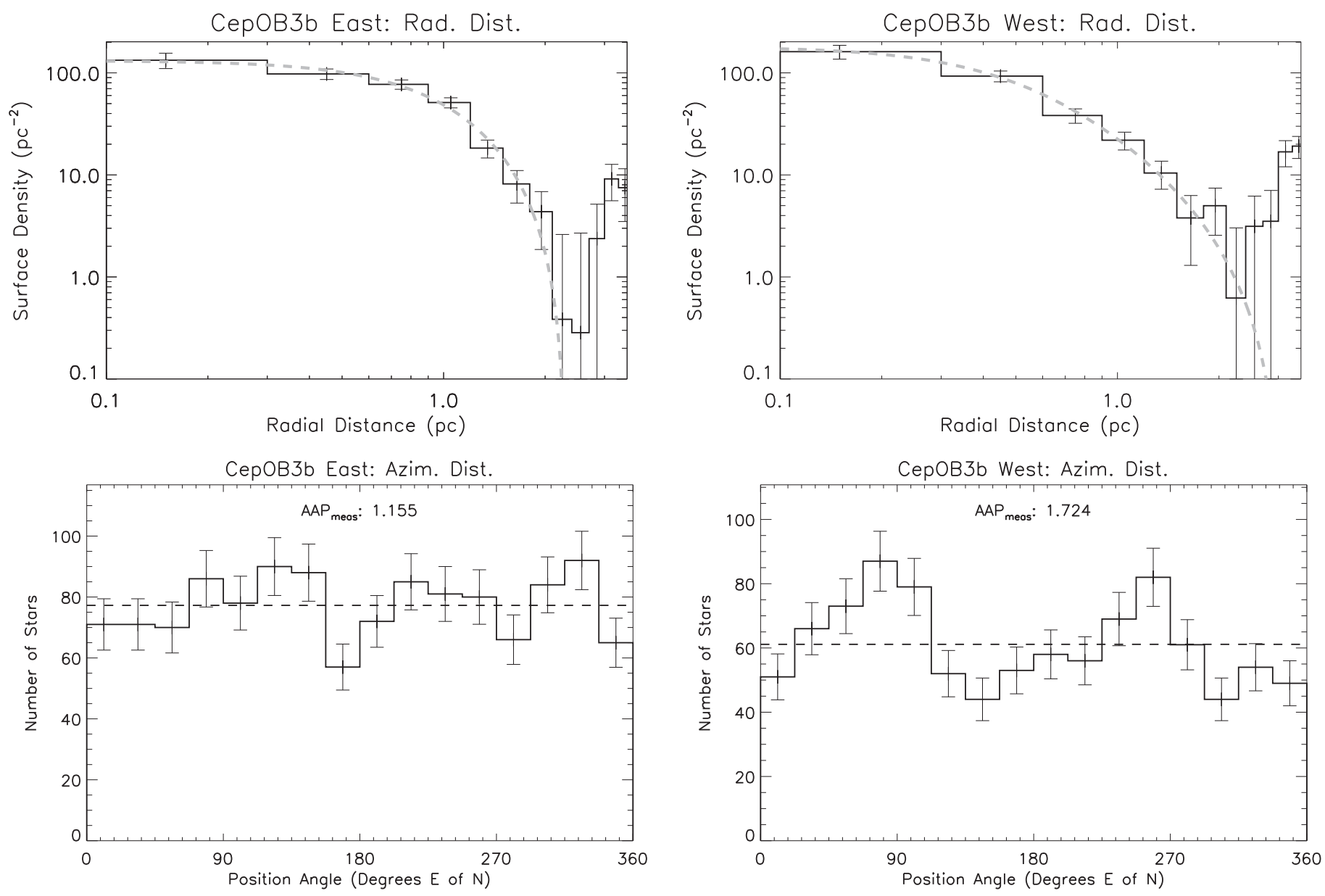

Figure 4. The best-fit 1962 King models. The top-left and -right plots are the uncorrected surface density vs. radial distance for the east and west subclusters, respectively. The bottom-left and -right plots are the number of stars vs. position angle for the east and west subclusters, respectively. The deviation of these from circular symmetry, which is given by the dashed line, determines the value of the azimuthal parameter (AAP; Gutermuth et al. 2005).

From the King model fits, the half-mass radius is 0.83 and $0.67 \mathrm{pc}$ for the east and west, respectively.

\subsection{The Velocity Structure of the Cep OB3b Cluster}

The position-velocity (PV) diagrams of the Cep OB3b cluster are shown in Figures 5 and 6; the positions of these stars in the subclusters are given in Figure 7. These figures indicate the lack of an apparent velocity gradient in decl. or R.A. for both subclusters and the entire region itself. The RVs in the west have a relatively flat distribution around the average RV. On the other hand, the east has a much wider spread of RVs but still lacks an RV gradient in R.A. or decl.

The average $\mathrm{RV}$ of the two subclusters are very similar, $-12.09 \mathrm{~km} \mathrm{~s}^{-1}$, with a standard error of the mean as $0.56 \mathrm{~km} \mathrm{~s}^{-1}$, and $-10.86 \mathrm{~km} \mathrm{~s}^{-1}$, with a standard error of the mean as $0.54 \mathrm{~km} \mathrm{~s}^{-1}$ for the east and west, respectively. These are within $2 \sigma$ of each other. The overall RV average is $-11.69 \mathrm{~km} \mathrm{~s}^{-1}$ with a standard error of the mean as $0.42 \mathrm{~km} \mathrm{~s}^{-1}$.

\subsection{The Velocity Dispersion of the Subclusters}

To constrain the velocity dispersions of the subclusters, we implement a Bayesian parameter estimation for the velocity dispersion in each subcluster. We determine the likelihood function using a Monte Carlo comparison to our measured

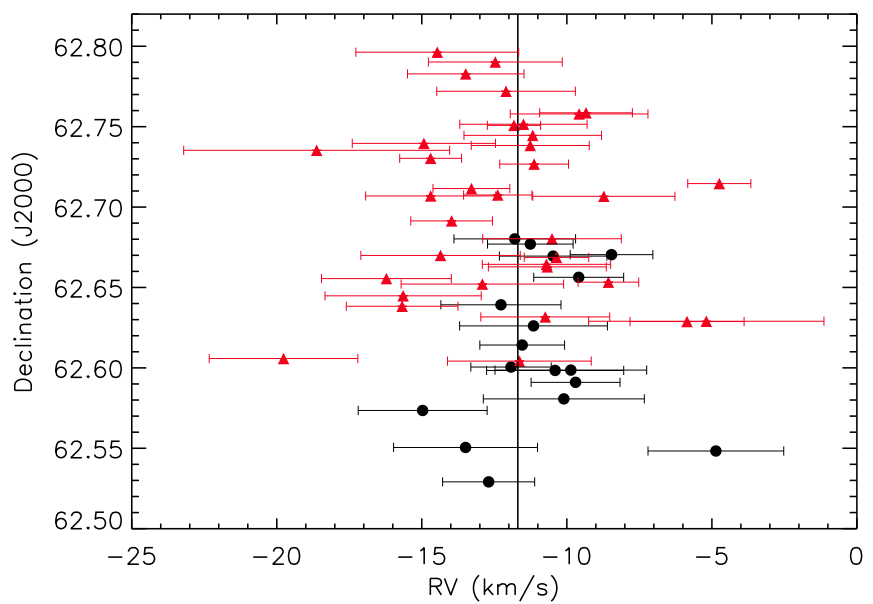

Figure 5. Decl. vs. RV for stars with youth indicators. The red triangles are objects in the east subcluster. The black circles are objects in the west subcluster.

velocity dispersions for the 35 and 17 stars of the eastern and western subclusters, respectively. The number of stars in each sub-cluster are determined by Figure 7, where member stars that are not potential binaries and have a $R \geqslant 5$ are shown with respect to the eastern and western subcluster radii. We start by 


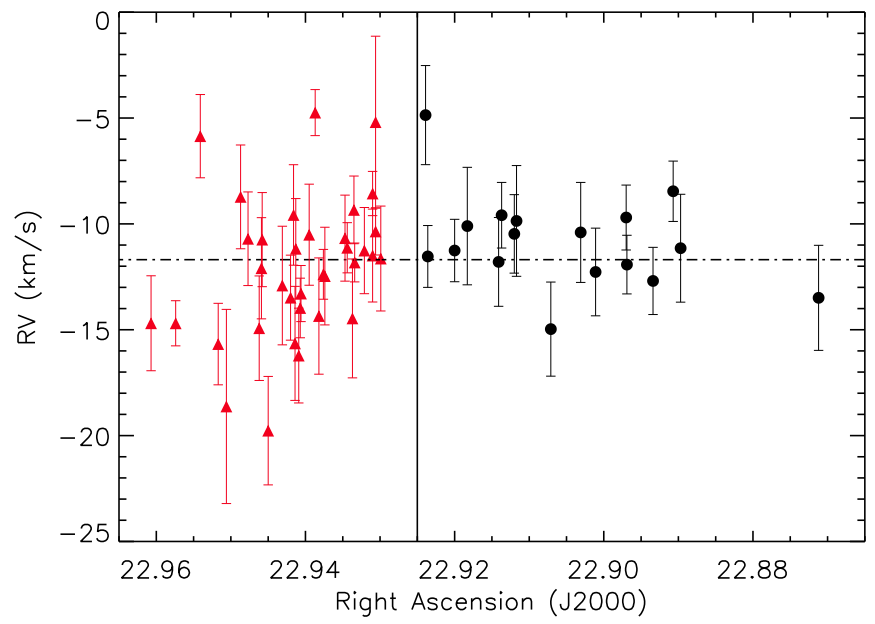

Figure 6. Radial velocity vs. R.A. for objects with youth indicators. The symbols and colors are the same as in Figure 5.

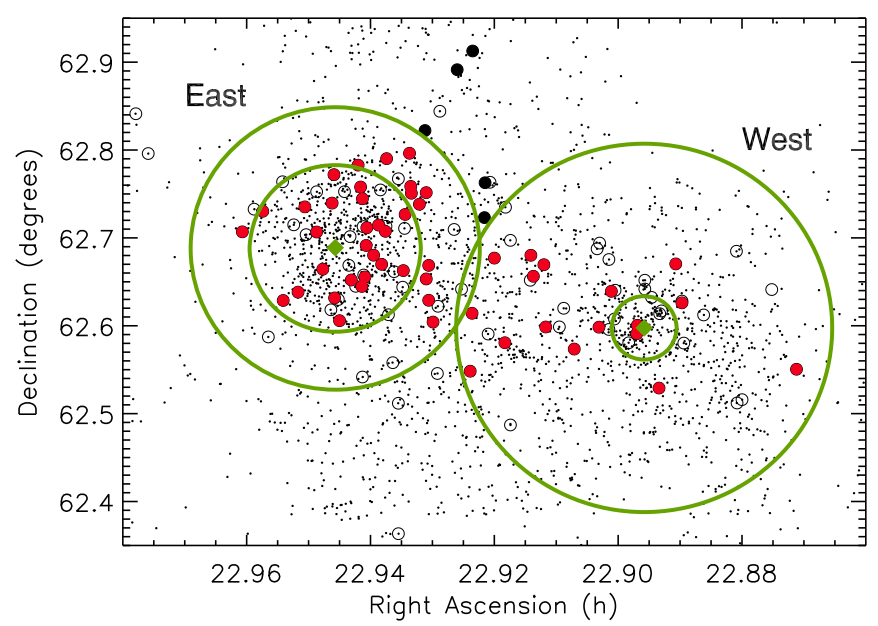

Figure 7. The black points are all the members of Cep OB3b (Allen et al. 2012), and the open circles have Hectoschelle spectra. The filled black circles are members with Hectoschelle spectra that survived the cuts described in Section 2, but fall outside the subcluster radii. The filled red circles are members with Hectoschelle spectra within the subcluster radii and are used in the kinematic analysis. The green diamonds are the centers of the respective subclusters and the green circles are the core and subcluster radii for each subcluster.

adopting 1D Gaussian velocity distributions centered on the RV of each subcluster; the distributions of the subclusters have independent velocity dispersions, $\sigma$. To calculate the velocity dispersion, $35 \mathrm{RVs}$ and $17 \mathrm{RVs}$ are randomly drawn from the Gaussian distribution of the east and west, respectively. We iterate this process, changing the width $\sigma$ of the Gaussian distribution. For each subcluster, the $\sigma$ starts at $0.05 \mathrm{~km} \mathrm{~s}^{-1}$ and increases in steps of $0.05 \mathrm{~km} \mathrm{~s}^{-1}$ until $\sigma=10 \mathrm{~km} \mathrm{~s}^{-1}$ is reached. The RVs were drawn 10,000 times for each value of $\sigma$.

To account for unresolved binaries, we adopt the approach of Cottaar et al. (2012) to add the effect of orbital motions. These would be cases without a double-lined spectrum where the velocities that we measure are those of the more luminous primary stars. We adopt three mass-based $\left(1 M_{\odot}, 0.75 M_{\odot}\right.$, $\left.0.5 M_{\odot}\right)$ log-normal velocity distributions. The solar-type distribution of absolute velocities has a log-normal width of 0.84 $\log _{10} \mathrm{~km} \mathrm{~s}^{-1}$ and a mean of $0.08 \log _{10} \mathrm{~km} \mathrm{~s}^{-1}$ (Cottaar et al. 2012).

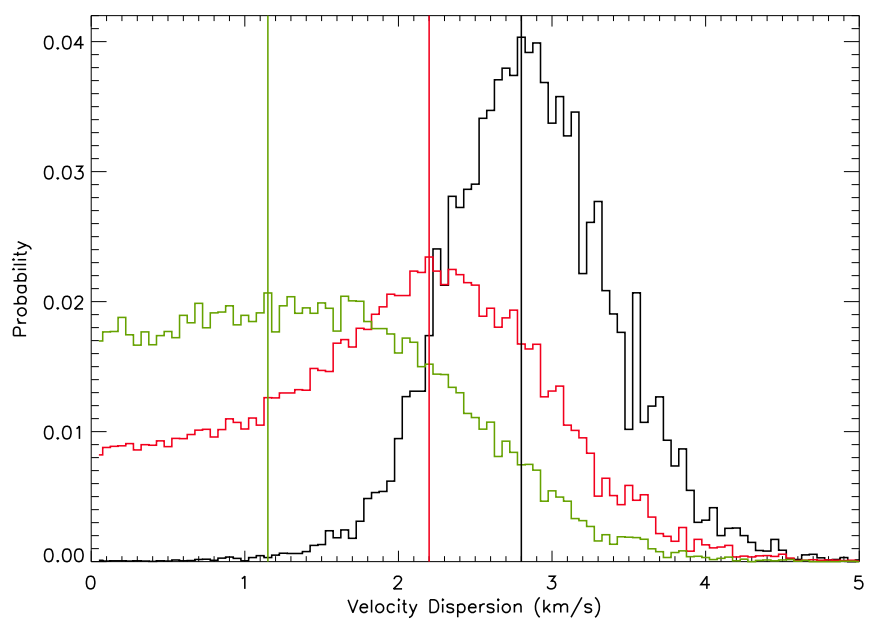

Figure 8. The PDF of the velocity distribution in the east subcluster. The black histogram is the PDF for a binary fraction of 0 with a peak velocity dispersion value of $2.8 \mathrm{~km} \mathrm{~s}^{-1}$, the red for a binary fraction of 0.5 with a peak velocity dispersion value of $2.2 \mathrm{~km} \mathrm{~s}^{-1}$, and the green for a binary fraction of 1.0 with a peak velocity dispersion value of $1.15 \mathrm{~km} \mathrm{~s}^{-1}$. The vertical lines represent the peak value of the distribution. Changing the binary fraction has a significant effect on the PDF of the velocity dispersion.

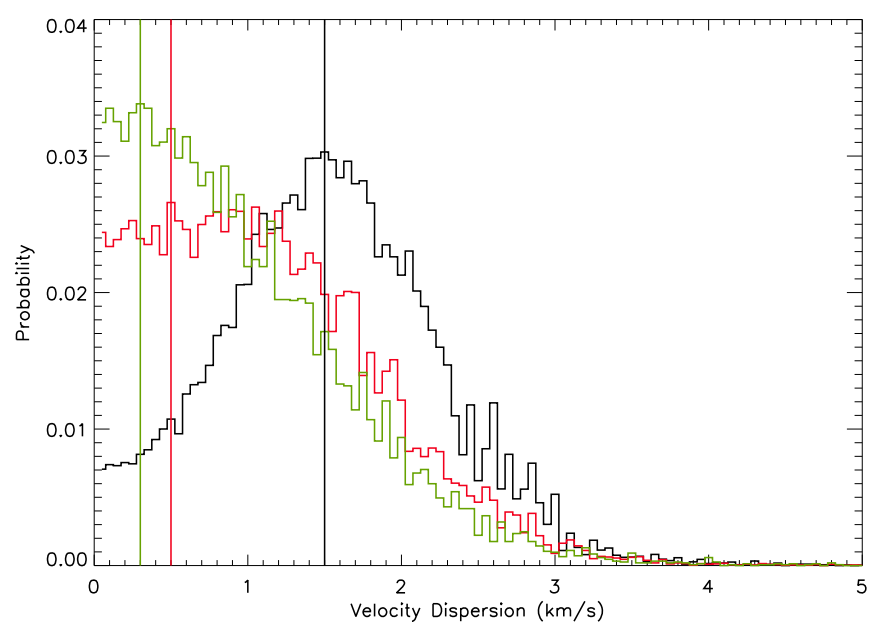

Figure 9. The PDF of the velocity distribution in the west subcluster. The black histogram is the PDF for a binary fraction of 0 with a peak velocity dispersion value of $1.5 \mathrm{~km} \mathrm{~s}^{-1}$, the red for a binary fraction of 0.5 with a peak velocity dispersion value of $0.5 \mathrm{~km} \mathrm{~s}^{-1}$, and the green for a binary fraction of 1.0 with a peak velocity dispersion value of $0.3 \mathrm{~km} \mathrm{~s}^{-1}$. The vertical lines represent the peak value of the distribution. Changing the binary fraction has little effect on the resulting velocity dispersion.

The $0.75 M_{\odot}\left(0.62 \log _{10} \mathrm{~km} \mathrm{~s}^{-1}\right.$ width and $0.35 \log _{10} \mathrm{~km} \mathrm{~s}^{-1}$ mean $)$ and $0.5 M_{\odot}\left(0.15 \log _{10} \mathrm{~km} \mathrm{~s}^{-1}\right.$ width and $0.52 \log _{10} \mathrm{~km} \mathrm{~s}^{-1}$ mean) distributions are scaled from the solar-type distribution by implementing Equation (3) in Cottaar et al. (2012) and using the orbital period distributions from Table 1 in Duchêne \& Kraus (2013). Using the spectral types from T. S. Allen et al. (2018, in preparation), we estimate the masses of the 35 (17) members and assign them to the appropriate mass-based binary velocity distribution. A total of 13 objects fall into the solar-type bin, 15 fall into the $0.75 M_{\odot}$ bin, and 24 fall into the $0.5 M_{\odot}$ bin.

We test three binary fractions: $0,0.5$, and 1 . Stars are randomly assigned a binary motion from the appropriate massbased Gaussian distribution. The typical FWHM of the crosscorrelation plots is $\sim 40 \mathrm{~km} \mathrm{~s}^{-1}$. We throw out binary motions 
Table 7

Potential Spectroscopic Binary Candidates

\begin{tabular}{|c|c|c|c|}
\hline 2MASS ID & R.A. (J2000) & Decl. (J2000) & $\overline{\mathrm{RV}} \pm \sigma\left(\mathrm{km} \mathrm{s}^{-1}\right)$ \\
\hline $\mathrm{J} 22553151+6253093$ & $22: 55: 32.52$ & 62:53:09.48 & $-22.13 \pm 4.20$ \\
\hline $\mathrm{J} 22550645+6241517$ & $22: 55: 06.47$ & $62: 41: 51.78$ & $15.38 \pm 2.25$ \\
\hline $\mathrm{J} 22560723+6245358$ & $22: 56: 07.23$ & $62: 45: 35.76$ & $-15.69 \pm 6.15$ \\
\hline $\mathrm{J} 22552722+6243290$ & $22: 55: 27.23$ & $62: 43: 28.90$ & $15.19 \pm 5.35$ \\
\hline $\mathrm{J} 22561571+6242509$ & $22: 56: 15.72$ & 62:42:51.08 & $-12.90 \pm 2.57$ \\
\hline J22560574+6242599 & $22: 56: 05.75$ & 62:42:59.74 & $6.90 \pm 2.83$ \\
\hline $\mathrm{J} 22570068+6245485$ & $22: 57: 00.70$ & 62:45:48.44 & $-20.45 \pm 10.68$ \\
\hline $\mathrm{J} 22563906+6245092$ & $22: 56: 39.04$ & $62: 45: 09.22$ & $36.56 \pm 1.55$ \\
\hline $\mathrm{J} 22564077+6245453$ & $22: 56: 40.74$ & $62: 45: 45.36$ & $-46.79 \pm 6.55$ \\
\hline $\mathrm{J} 22561377+6240505$ & $22: 56: 13.76$ & $62: 40: 50.60$ & $-1.17 \pm 6.70$ \\
\hline $\mathrm{J} 22554154+6239030$ & $22: 55: 41.54$ & 62:39:03.11 & $-21.34 \pm 2.41$ \\
\hline $\mathrm{J} 22570919+6233337$ & $22: 57: 09.25$ & $62: 33: 33.59$ & $7.32 \pm 8.98$ \\
\hline $\mathrm{J} 22562445+6233559$ & $22: 56: 24.47$ & 62:33:55.89 & $-32.36 \pm 4.09$ \\
\hline $\mathrm{J} 22555063+6228416$ & $22: 55: 50.61$ & $62: 28: 41.52$ & $-18.72 \pm 5.83$ \\
\hline $\mathrm{J} 22545686+6234128$ & $22: 54: 56.86$ & $62: 34: 12.58$ & $-9.17 \pm 4.53$ \\
\hline $\mathrm{J} 22545903+6237177$ & $22: 54: 59.03$ & $62: 37: 17.82$ & $-18.48 \pm 3.74$ \\
\hline $\mathrm{J} 22545364+6234031$ & $22: 54: 53.61$ & 62:34:03.10 & $-18.92 \pm 3.56$ \\
\hline $\mathrm{J} 22535527+6233218$ & $22: 53: 55.26$ & $62: 33: 21.76$ & $-21.93 \pm 6.62$ \\
\hline $\mathrm{J} 22542405+6235158$ & $22: 54: 24.04$ & 62:35:15.95 & $-1.88 \pm 4.77$ \\
\hline $\mathrm{J} 22523087+6231116$ & $22: 52: 30.84$ & $62: 31: 11.45$ & $-13.52 \pm 6.06$ \\
\hline $\mathrm{J} 22534360+6235322$ & $22: 53: 43.60$ & $62: 35: 32.20$ & $-38.99 \pm 6.97$ \\
\hline $\mathrm{J} 22540438+6235548$ & $22: 54: 04.37$ & $62: 35: 54.68$ & $-11.81 \pm 5.33$ \\
\hline $\mathrm{J} 22532966+6234051$ & $22: 53: 29.64$ & $62: 34: 05.00$ & $-21.55 \pm 2.01$ \\
\hline $\mathrm{J} 22535723+6236506$ & $22: 53: 57.24$ & $62: 36: 50.70$ & $-58.37 \pm 2.01$ \\
\hline $\mathrm{J} 22531785+6234592$ & $22: 53: 17.86$ & 62:34:59.10 & $-21.64 \pm 6.49$ \\
\hline $\mathrm{J} 22533413+6238199$ & $22: 53: 33.19$ & $62: 38: 20.55$ & $-28.19 \pm 4.50$ \\
\hline $\mathrm{J} 22540632+6243500$ & $22: 54: 06.33$ & 62:43:50.09 & $30.02 \pm 1.32$ \\
\hline $\mathrm{J} 22541162+6242285$ & $22: 54: 11.63$ & $62: 42: 28.55$ & $-28.24 \pm 10.85$ \\
\hline $\mathrm{J} 22550261+6251081$ & $22: 55: 01.39$ & $62: 51: 11.83$ & $-27.97 \pm 3.78$ \\
\hline $\mathrm{J} 22542910+6254472$ & $22: 54: 29.07$ & 62:54:47.07 & $-24.82 \pm 5.41$ \\
\hline $\mathrm{J} 22545316+6250085$ & $22: 54: 53.16$ & $62: 50: 08.35$ & $-25.52 \pm 4.63$ \\
\hline $\mathrm{J} 22542993+6240335$ & $22: 54: 29.92$ & $62: 40: 33.57$ & $-8.10 \pm 7.02$ \\
\hline $\mathrm{J} 22543513+6245431$ & $22: 54: 35.14$ & $62: 45: 43.20$ & $-39.69 \pm 7.01$ \\
\hline $\mathrm{J} 22550915+6253570$ & $22: 55: 09.14$ & $62: 53: 57.12$ & $-31.49 \pm 7.90$ \\
\hline $\mathrm{J} 22552486+6246446$ & $22: 55: 24.87$ & $62: 46: 44.63$ & $33.22 \pm 3.33$ \\
\hline $\mathrm{J} 22554749+6241576$ & $22: 55: 47.49$ & 62:41:57.68 & $-11.94 \pm 4.73$ \\
\hline $\mathrm{J} 22564371+6242017$ & $22: 56: 43.72$ & 62:42:01.68 & $-12.56 \pm 5.07$ \\
\hline $\mathrm{J} 22563865+6237140$ & $22: 56: 38.68$ & $62: 37: 14.29$ & $-31.93 \pm 4.52$ \\
\hline $\mathrm{J} 22555006+6223083$ & $22: 55: 50.15$ & $62: 23: 08.25$ & $-16.35 \pm 4.04$ \\
\hline $\mathrm{J} 22551657+6232522$ & $22: 55: 16.59$ & $62: 32: 52.12$ & $-14.09 \pm 3.57$ \\
\hline $\mathrm{J} 22522558+6222059$ & $22: 52: 25.60$ & 62:22:05.91 & $-14.94 \pm 6.56$ \\
\hline $\mathrm{J} 22543423+6235555$ & $22: 54: 34.20$ & 62:35:55.46 & $2.70 \pm 5.57$ \\
\hline $\mathrm{J} 22531578+6235262$ & $22: 53: 15.78$ & $62: 35: 26.13$ & $-12.71 \pm 15.25$ \\
\hline $\mathrm{J} 22533664+6236489$ & $22: 53: 36.65$ & $62: 36: 48.99$ & $-13.82 \pm 7.32$ \\
\hline $\mathrm{J} 22533869+6237171$ & $22: 53: 38.67$ & $62: 37: 17.23$ & $11.21 \pm 7.64$ \\
\hline $\mathrm{J} 22550760+6244452$ & $22: 55: 08.12$ & $62: 44: 57.93$ & $35.31 \pm 2.45$ \\
\hline $\mathrm{J} 22553847+6255182$ & $22: 55: 37.59$ & $62: 55: 19.18$ & $49.39 \pm 7.66$ \\
\hline $\mathrm{J} 22555591+6252385$ & $22: 55: 55.86$ & $62: 52: 38.46$ & $-15.80 \pm 7.92$ \\
\hline $\mathrm{J} 22562031+6254458$ & $22: 56: 20.39$ & 62:54:46.05 & $-17.91 \pm 1.62$ \\
\hline $\mathrm{J} 22550959+6244210$ & 22:55:09.61 & $62: 44: 21.04$ & $-7.00 \pm 4.12$ \\
\hline $\mathrm{J} 22561978+6248022$ & $22: 56: 19.81$ & 62:48:02.17 & $-2.35 \pm 3.75$ \\
\hline $\mathrm{J} 22563678+6246031$ & $22: 56: 36.82$ & 62:46:02.94 & $-8.02 \pm 7.08$ \\
\hline $\mathrm{J} 22562134+6243257$ & $22: 56: 21.33$ & $62: 43: 25.71$ & $-12.87 \pm 3.89$ \\
\hline $\mathrm{J} 22565035+6242485$ & $22: 56: 50.36$ & $62: 42: 48.58$ & $-15.15 \pm 4.60$ \\
\hline $\mathrm{J} 22570134+6242133$ & $22: 57: 01.36$ & $62: 42: 13.43$ & $-12.87 \pm 13.56$ \\
\hline $\mathrm{J} 22563127+6240588$ & $22: 56: 31.26$ & $62: 40: 58.71$ & $-11.92 \pm 4.30$ \\
\hline $\mathrm{J} 22542460+6225053$ & $22: 54: 24.63$ & $62: 25: 05.22$ & $-25.93 \pm 1.95$ \\
\hline $\mathrm{J} 22535945+6235102$ & $22: 53: 59.42$ & $62: 35: 10.21$ & $53.78 \pm 3.16$ \\
\hline $\mathrm{J} 22535969+6236320$ & $22: 53: 59.69$ & $62: 36: 32.03$ & $-22.27 \pm 4.59$ \\
\hline $\mathrm{J} 22531574+6234357$ & $22: 53: 15.71$ & $62: 34: 35.77$ & $-41.03 \pm 3.84$ \\
\hline $\mathrm{J} 22504792+6233227$ & $22: 50: 47.94$ & $62: 33: 22.62$ & $-33.24 \pm 2.58$ \\
\hline $\mathrm{J} 22542135+6248095$ & $22: 54: 21.35$ & $62: 48: 09.36$ & $-9.31 \pm 1.12$ \\
\hline $\mathrm{J} 22550804+6244252$ & $22: 55: 08.05$ & $62: 44: 25.41$ & $-5.57 \pm 1.83$ \\
\hline $\mathrm{J} 22554785+6252037$ & $22: 55: 47.84$ & $62: 52: 03.65$ & $-13.88 \pm 7.14$ \\
\hline
\end{tabular}


Table 7

(Continued)

\begin{tabular}{|c|c|c|c|}
\hline 2MASS ID & R.A. (J2000) & Decl. (J2000) & $\mathrm{RV} \pm \sigma\left(\mathrm{km} \mathrm{s}^{-1}\right)$ \\
\hline $\mathrm{J} 22561978+6248022$ & $22: 56: 19.81$ & $62: 48: 02.17$ & $-13.84 \pm 2.83$ \\
\hline $\mathrm{J} 22552722+6243290$ & $22: 55: 27.23$ & $62: 43: 28.90$ & $-11.74 \pm 4.74$ \\
\hline $\mathrm{J} 22563906+6245092$ & $22: 56: 39.04$ & $62: 45: 09.22$ & $20.64 \pm 2.42$ \\
\hline $\mathrm{J} 22562134+6243257$ & $22: 56: 21.34$ & $62: 43: 25.71$ & $-12.90 \pm 5.30$ \\
\hline $\mathrm{J} 22565123+6243197$ & $22: 56: 51.24$ & $62: 43: 19.74$ & $-11.04 \pm 3.76$ \\
\hline $\mathrm{J} 22565692+6236389$ & $22: 56: 56.91$ & $62: 36: 38.80$ & $-14.70 \pm 10.37$ \\
\hline $\mathrm{J} 22565217+6226391$ & $22: 56: 52.18$ & $62: 26: 39.07$ & $3.96 \pm 4.36$ \\
\hline $\mathrm{J} 22555686+6234332$ & $22: 55: 56.85$ & $62: 34: 33.24$ & $20.33 \pm 3.63$ \\
\hline $\mathrm{J} 22555006+6223083$ & $22: 55: 50.06$ & $62: 23: 08.26$ & $-14.02 \pm 4.15$ \\
\hline $\mathrm{J} 22550975+6239074$ & $22: 55: 09.71$ & $62: 39: 07.24$ & $29.33 \pm 6.39$ \\
\hline $\mathrm{J} 22545903+6237177$ & 22:54:59.04 & $62: 37: 17.82$ & $0.88 \pm 10.67$ \\
\hline $\mathrm{J} 22542760+6225270$ & $22: 54: 27.60$ & $62: 25: 26.88$ & $-5.50 \pm 8.75$ \\
\hline $\mathrm{J} 22541824+6226460$ & $22: 54: 18.24$ & $62: 26: 45.93$ & $5.93 \pm 8.75$ \\
\hline $\mathrm{J} 22542405+6235158$ & $22: 54: 24.05$ & $62: 35: 15.95$ & $-14.11 \pm 2.62$ \\
\hline $\mathrm{J} 22523242+6227422$ & $22: 52: 32.41$ & $62: 27: 42.40$ & $-3.32 \pm 15.06$ \\
\hline $\mathrm{J} 22530063+6237581$ & $22: 53: 00.63$ & $62: 37: 58.14$ & $30.89 \pm 2.00$ \\
\hline $\mathrm{J} 22531297+6238444$ & $22: 53: 13.00$ & $62: 38: 44.41$ & $30.05 \pm 1.47$ \\
\hline $\mathrm{J} 22543623+6241336$ & $22: 54: 36.24$ & $62: 41: 33.81$ & $-39.31 \pm 2.33$ \\
\hline $\mathrm{J} 22570134+6242133$ & $22: 57: 01.35$ & $62: 42: 13.39$ & $11.91 \pm 4.25$ \\
\hline $\mathrm{J} 22555010+6237443$ & $22: 55: 50.10$ & $62: 37: 44.32$ & $-9.30 \pm 2.45$ \\
\hline $\mathrm{J} 22570919+6233337$ & $22: 57: 09.20$ & $62: 33: 33.75$ & $1.81 \pm 10.08$ \\
\hline $\mathrm{J} 22561318+6235331$ & $22: 56: 13.18$ & $62: 35: 33.15$ & $12.59 \pm 2.08$ \\
\hline $\mathrm{J} 22553370+6233093$ & $22: 55: 33.70$ & $62: 33: 09.34$ & $-25.55 \pm 1.44$ \\
\hline $\mathrm{J} 22553606+6238563$ & $22: 55: 36.25$ & 62:39:02.35 & $-6.12 \pm 5.42$ \\
\hline $\mathrm{J} 22545364+6234031$ & $22: 54: 53.64$ & $62: 34: 03.10$ & $-18.24 \pm 2.54$ \\
\hline $\mathrm{J} 22535527+6233218$ & $22: 53: 55.27$ & $62: 33: 21.85$ & $-16.36 \pm 8.71$ \\
\hline $\mathrm{J} 22541270+6235379$ & $22: 54: 12.70$ & $62: 35: 37.95$ & $-23.91 \pm 6.71$ \\
\hline $\mathrm{J} 22530063+6237581$ & $22: 53: 00.64$ & $62: 37: 58.19$ & $-22.68 \pm 0.96$ \\
\hline $\mathrm{J} 22533869+6237171$ & $22: 53: 38.69$ & $62: 37: 17.11$ & $-12.45 \pm 4.94$ \\
\hline $\mathrm{J} 22530099+6243548$ & 22:53:01.00 & $62: 43: 54.82$ & $1.69 \pm 4.33$ \\
\hline $\mathrm{J} 22550959+6244210$ & $22: 55: 09.60$ & $62: 44: 21.03$ & $-21.37 \pm 4.58$ \\
\hline $\mathrm{J} 22550561+6244055$ & $22: 55: 05.61$ & $62: 44: 05.56$ & $-26.24 \pm 7.41$ \\
\hline $\mathrm{J} 22552722+6243290$ & $22: 55: 27.22$ & $62: 43: 29.03$ & $-14.88 \pm 4.14$ \\
\hline $\mathrm{J} 22563677+6255599$ & $22: 56: 36.77$ & $62: 55: 59.94$ & $-16.43 \pm 4.40$ \\
\hline $\mathrm{J} 22562134+6243257$ & $22: 56: 21.34$ & $62: 43: 25.72$ & $6.65 \pm 9.85$ \\
\hline
\end{tabular}

Table 8

King (1962) Fit Results

\begin{tabular}{|c|c|c|c|c|c|c|c|c|}
\hline Subcluster & Disk Fraction & $\begin{array}{c}A \\
\left(\mathrm{pc}^{-2}\right)^{1}\end{array}$ & $\begin{array}{l}R_{\text {core }} \\
(\mathrm{pc})\end{array}$ & $\begin{array}{l}R_{\text {tidal }} \\
(\mathrm{pc})\end{array}$ & $\mathrm{AAP}_{\text {meas }}$ & $\begin{array}{l}\text { Number of } \\
\text { Members }\end{array}$ & $\begin{array}{c}\Sigma_{\text {mean }} \\
\text { stars }\left(\mathrm{pc}^{-2}\right)\end{array}$ & $\left.\begin{array}{c}\Sigma_{\text {peak }} \\
(\text { stars pc } \\
-2\end{array}\right)$ \\
\hline$\overline{\text { East }}$ & 0.32 & $541 \pm 202$ & $1.36 \pm 0.30$ & $2.32 \pm 0.19$ & 1.155 & 809 & 240 & 521 \\
\hline East & 0.39 & $541 \pm 202$ & $1.36 \pm 0.30$ & $2.32 \pm 0.19$ & 1.155 & 664 & 197 & 428 \\
\hline West & 0.44 & $258 \pm 42$ & $0.52 \pm 0.11$ & $3.1 \pm 1.0$ & 1.724 & 501 & 220 & 342 \\
\hline West & 0.53 & $258 \pm 42$ & $0.52 \pm 0.11$ & $3.1 \pm 1.0$ & 1.724 & 416 & 182 & 284 \\
\hline
\end{tabular}

Note.

${ }^{1}$ This value has not been scaled to correct for the disk fraction.

that were greater than this because we are able to detect them in the initial RV extraction. Finally, to take into account the uncertainties in the RV measurements, we add velocities drawn from randomly sampling Gaussian distributions with $\sigma$ equal to the uncertainties of the measured velocities.

We use the fraction of times the 10,000 simulated velocity distributions are within $0.1 \mathrm{~km} \mathrm{~s}^{-1}$ of the actual velocity distribution to create the posterior probability density function (PDF) of the velocity dispersions. The PDFs of the velocity dispersions for the east and west are shown in Figures 8 and 9, respectively. The expectation values for the two subclusters for a binary fraction of 0.5 with $\pm 1 \sigma$ confidence limits are
$1.91 \pm 1.0 \mathrm{~km} \mathrm{~s}^{-1}$ for the east and $1.1_{-0.5}^{+0.8} \mathrm{~km} \mathrm{~s}^{-1}$ for the west. The peak values for these distributions are $2.2 \pm 1.0 \mathrm{~km} \mathrm{~s}^{-1}$ and $0.5_{-0.5}^{+0.8} \mathrm{~km} \mathrm{~s}^{-1}$ for the east and west, respectively.

We explore the influence of the binary fractions on the velocity dispersion of the eastern subcluster in Figure 8 . The binary fraction changes the resulting velocity dispersion for the east. The velocity dispersion is centered at $2.8 \mathrm{~km} \mathrm{~s}^{-1}$ for a binary fraction of zero. As the binary fraction increases the distribution widens and flattens moving closer to $0 \mathrm{~km} \mathrm{~s}^{-1}$. This demonstrates that accounting for unresolved binaries is an important step to probing an accurate kinematical survey of young clusters. The western subcluster probability distribution 

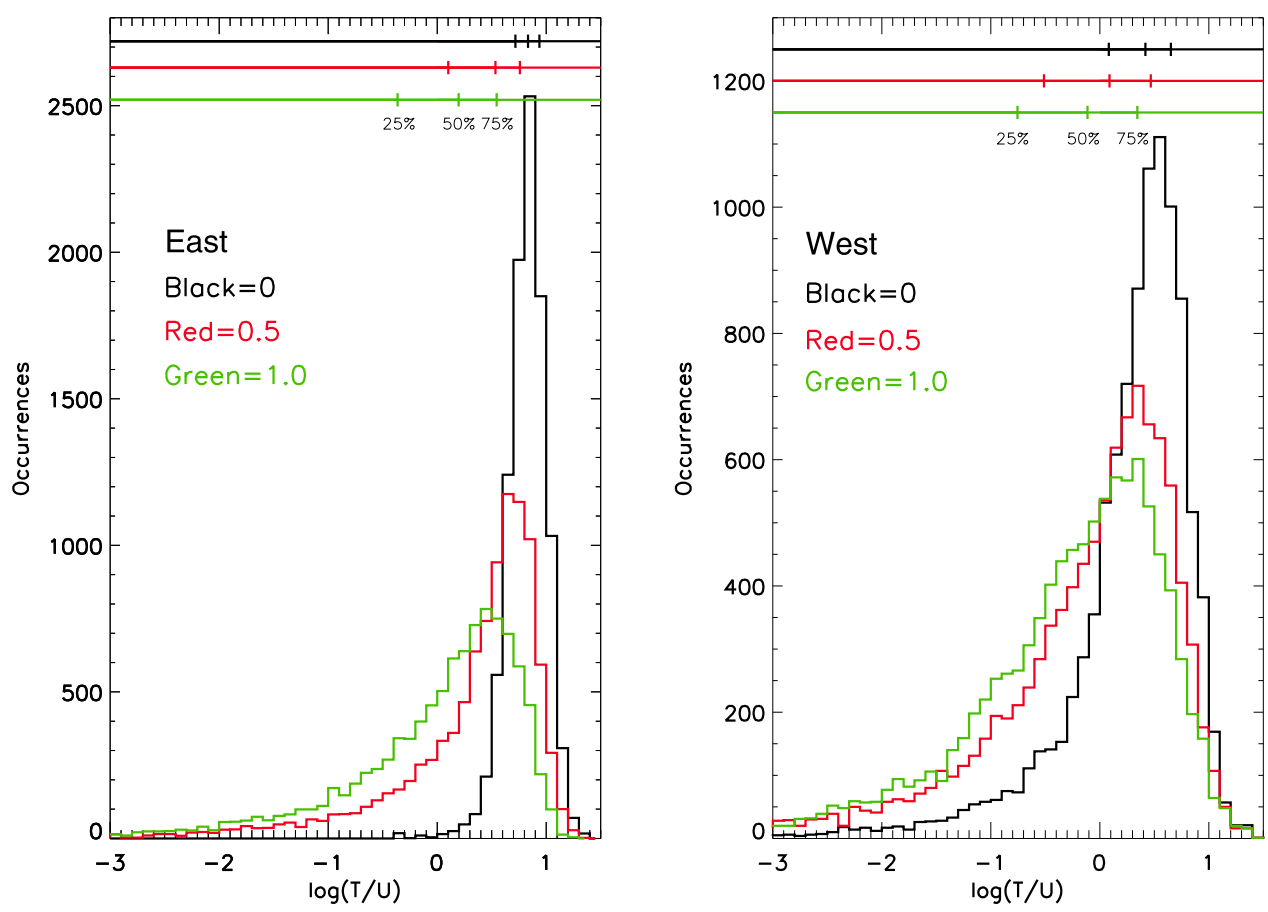

Figure 10. Plot of $\log (T /|U|)$ for the east (left) and west (right) subclusters from the posterior probability distribution accounting for a changing binary fraction. The black histogram for a binary fraction of 0 , the red for a binary fraction of 0.5 , and the green for a binary fraction of 1.0. The horizontal lines the top are marked for quartile positions in the distributions. In both cases, the peak $\log (T /|U|)$ evolves to a flatter and more bound distribution as the binary fraction increases.

(Figure 9) peaks at $1.5 \mathrm{~km} \mathrm{~s}^{-1}$ for zero binaries and moves closer to zero for binary fractions of 0.5 and 1.0, flattening and widening as in the east (Figure 8).

Jeffries et al. (2014) uses a maximum likelihood technique to account for unresolved binaries in Gamma Vel, which is adopted from Cottaar et al. (2012). Applying this approach to the Cep OB3b data, with the binary fraction set to 0.5 and the masses at $0.5 M_{\odot}$, results in an intrinsic Gaussian dispersion of $1.62 \pm 0.75 \mathrm{~km} \mathrm{~s}^{-1}$ and $0.78 \pm 0.60 \mathrm{~km} \mathrm{~s}^{-1}$ for the east and west, respectively. If the binary fraction is set to 0 , then the dispersions increase to $2.54 \pm 0.44 \mathrm{~km} \mathrm{~s}^{-1}$ and $0.8 \pm 0.61 \mathrm{~km} \mathrm{~s}^{-1}$ for the east and west, respectively. The results of the velocity dispersions for the different binary fractions agree at the $1 \sigma$ level between our Bayesian analysis and the maximum likelihood technique.

\subsection{The Total Energy of the Eastern and Western Subclusters}

We use Equation (27) in King (1962) and combine the parameter fits of the core radius, subcluster radius, and the number of stars from Section 4.2 to determine the potential energies of the subclusters. The number of stars and the subcluster radius are kept constant and only the core radius was allowed to vary because it has the largest impact on the potential energy. The value of $A$, the surface density of stars at the center of the cluster, is adjusted to keep the number of stars fixed to the total number of stars estimated in Section 4.2; the uncertainty in this number is taken into account by repeating the calculation with the two different disk fractions. We ran the calculation 10,000 times to create a PDF for the potential energies of each subcluster.

We adopt the PDFs of the subcluster velocity dispersions to derive kinetic energies of the subclusters 10,000 times. The kinetic energy PDFs assume a symmetric three-dimensional velocity dispersion with the $\sigma$ for the two directions in the plane of the sky equal to that in the radial direction. Thus, the total kinetic energy is given by $3 / 2 M_{\text {stars }} \sigma^{2}$. Note that the mass of stars $\left(0.5 M_{\odot} \times N_{\text {members }}\right)$ appears linearly in the denominator of $T /|U|$ because the mass in the kinetic energy cancels a mass in the potential energy.

For each of the 10,000 iterations, we combine the kinetic and potential energy PDFs for each subcluster to determine a final PDF of 10,000 points, i.e., $\log (T /|U|)$. We plot the quartiles of this distribution in Figure 10. The change in the resulting value of $\log (T /|U|)$ between the two disk fractions for both subclusters is insignificant, and therefore we show one disk fraction for each subcluster in Figures 10 and 11. Adopting a binary fraction of 0.5 , the east $\log (T /|U|)$ has a mean value at 0.3 and a value at the peak of the distribution of 0.6 (Figure 11). This implies that the eastern subcluster is unbound and in a state of expansion. A binary fraction of 0 results in log $(T /|U|)>0$, also implying an unbound, expanding state. We note that unbound simply means that $\log (T /|U|)>0$; it is possible that parts of the subcluster may be bound as we will discuss later. A binary fraction of 1 has a mean value in a subvirial, bound state with a probability of $61 \%$ of being unbound.

For the west subcluster, adopting a binary fraction of 0.5 , results in a mean of $\log (T /|U|) \sim-0.16$ with a large range of outcomes and a peak value at 0.3 (Figure 11). The mean value falls into an approximate virial state with a $55 \%$ chance of an unbound state. A binary fraction of 0 results in a subvirial state with a $79 \%$ probability of being unbound. For a binary fraction of 1 , the west is subvirial with roughly equal chances of being bound or unbound. From these results, the west appears to have a velocity dispersion very close to zero, depending on the binary fraction. 

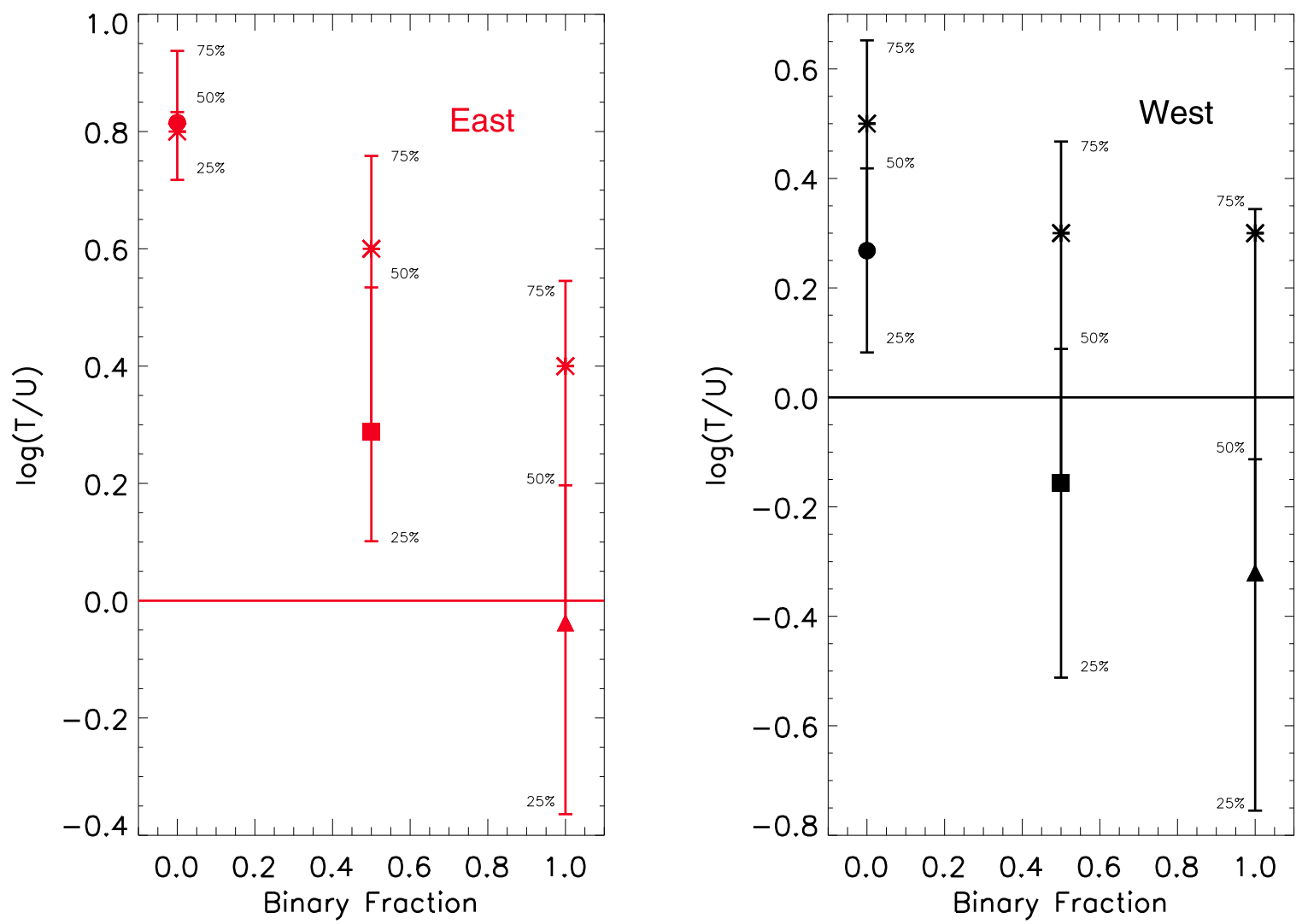

Figure 11. Plot of $\log (T /|U|)$ for both subclusters. The eastern subcluster is represented in red (left), and the west subcluster is represented in black (right) as a function of the adopted binary fraction. The horizontal line indicates $\log (T /|U|)=0.0$; this is the dividing line between a bound and unbound cluster. The circles, squares, and triangles are the mean value for each distribution in Figure 10, and the overlaid asterisks are the values at the peaks of the distributions in Figure 10. The quartile positions that correspond to those in Figure 10 are indicated.

It is important to recall that not all of the gas mass has been expelled from Cep OB3b even though it is currently in the gas dispersal phase. Gas remains in both subclusters (see Allen et al. 2012, Figure 4). We calculate the amount of gas mass in the subclusters based on the 2MASS extinction map. Allen et al. (2014) calculated that there was roughly $1 A_{v}$ of foreground extinction along the line of sight to Cep OB $3 \mathrm{~b}$. We integrate the amount of extinction greater than 2 mag inside each subcluster radius and adopt a distance of $819 \mathrm{pc}$ from Gaia DR2 as explained below in Section 4.6. This results in $\sim 136 M_{\odot}$ and $\sim 697 M_{\odot}$ of gas mass in the east and west, respectively. The gas measured in the east is concentrated near the edge of the subcluster and is not centrally located. In contrast, the gas mass in the west is concentrated inside the core radius. The west's mass is dominated by the gas, up to $77 \%$, and because it is centrally concentrated, it is necessary to include the gas mass in the potential energy. Accounting for the gas mass, the $\log (T /|U|)$ values drop by $\sim 0.4$, resulting in a subvirial, bound state, which we adopt as the more accurate kinematical result.

\subsection{The Distance to Cep OB3b and the Proper Motions of the Subclusters}

We cross-matched the Gaia DR2 catalog (Marrese et al. 2018) with Cep OB3b members with youth indicators (Allen et al. 2012) using their 2MASS IDs. We calculate the average proper motion vector components in R.A. $\left(\mu_{\alpha} *\right)$ and decl. $\left(\mu_{\delta}\right)$, where $\mu_{\alpha} *$ is $\mu_{\alpha} \times \cos (\delta)$. We use an R.A. of 22:54:48 to split
Table 9

GAIA DR2 Proper Motions

\begin{tabular}{lccc}
\hline \hline Subcluster & $\mu_{\alpha}\left(\mathrm{mas} \mathrm{yr}^{-1}\right)$ & $\mu_{\delta}\left(\mathrm{mas} \mathrm{yr}^{-1}\right)$ & $\mu_{\text {total }}\left(\mathrm{mas} \mathrm{yr}^{-1}\right)$ \\
\hline East & $-0.59 \pm 0.02$ & $-2.32 \pm 0.02$ & $2.34 \pm 0.02$ \\
West & $-1.25 \pm 0.03$ & $-2.78 \pm 0.02$ & $2.86 \pm 0.03$ \\
Cep OB3b & $-0.69 \pm 0.02$ & $-2.44 \pm 0.02$ & $2.46 \pm 0.02$ \\
\hline
\end{tabular}

the stars into the two subclusters. We perform a weighted fit of a Gaussian to the distributions of proper motions. We included proper motions between -10 and 10 mas yr$^{-1}$ in bins of $0.2 \mathrm{mas} \mathrm{yr}^{-1}$. The weight in each bin is given by the number of stars. The uncertainty in the mean proper motion is given by the Gaussian width normalized by the square root of the number of stars in each subcluster (696 and 370 for the east and west, respectively). The east has proper motion components of

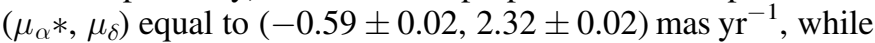
the west subcluster has proper motion components of $(-1.25 \pm 0.02,-2.78 \pm 0.02) \mathrm{mas} \mathrm{yr}^{-1}$ (Figure 12). The total cluster has proper motion components of $(0.69 \pm 0.02$, $-2.44 \pm 0.02) \mathrm{mas}^{-1} \mathrm{yr}^{-1}$. These values agree at the $1 \sigma$ level with Kuhn et al. (2018). Figure 13 shows the direction of the proper motions of each subcluster with the average proper motion of the total cluster removed (also see Table 9).

To determine a distance to Cep OB $3 \mathrm{~b}$, we used the cluster members with less than $20 \%$ uncertainty in their parallax measurements and nearby companions in the 2MASS point 


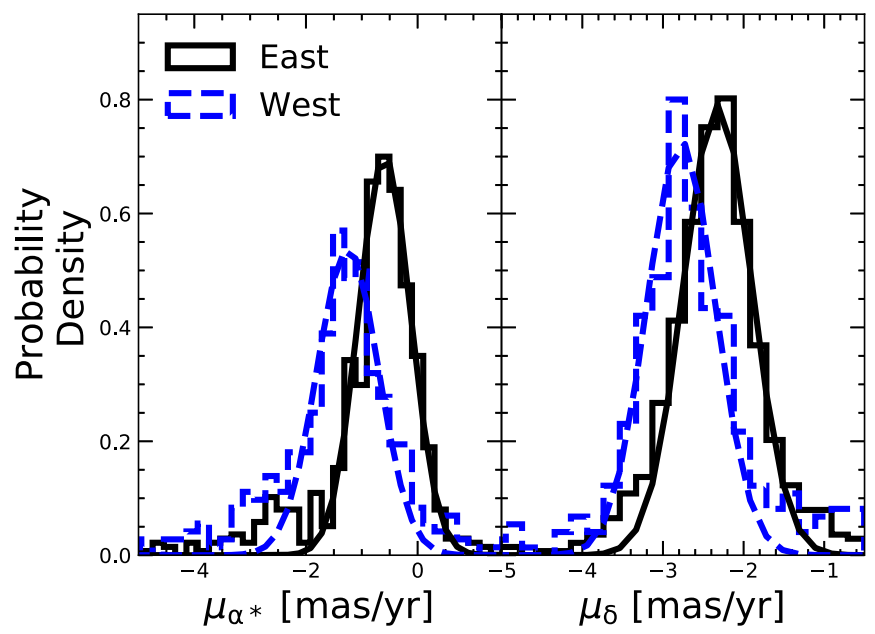

Figure 12. Histograms of the member proper motions in R.A. (left) and decl. (right) for the east (solid black) and west (dashed blue) subclusters. The bin sizes are 0.4 mas $\mathrm{yr}^{-1}$. The peaks of the probability densities show that the two subclusters have different values of proper motion components: $\mu_{\alpha}=$ $-0.59 \pm 0.02\left(\mathrm{mas} \mathrm{yr}^{-1}\right)$ and $\mu_{\delta}=-2.32 \pm 0.02\left(\mathrm{mas} \mathrm{yr}^{-1}\right)$ for the east and $\mu_{\alpha}=-1.25 \pm 0.03\left(\operatorname{mas~} \mathrm{yr}^{-1}\right)$ and $\mu_{\delta}=-2.78 \pm 0.02\left(\operatorname{mas~yr}^{-1}\right)$ for the west. The Gaussian fits to the distributions are shown.

source catalog; the resulting histogram of parallaxes shows a clear peak (Figure 14). The parallax value correction of $0.029{\text { mas } \mathrm{yr}^{-1}}^{-1}$ was used (Lindegren et al. 2018); a distance of $819 \pm 16 \mathrm{pc}$ is found after correcting for the zero-point offset of the Gaia parallaxes. The recommended DR2 filtering to remove objects with a high excess of astrometric noise was also applied to our sample. Previous measurements have been made for the distance to Cep OB3b: $580 \pm 60 \mathrm{pc}$ using an age and distance ladder (Littlefair et al. 2010), 700 pc from a maser parallax-determined distance for Cepheus A (Moscadelli et al. 2009), 725 pc from near-IR color-magnitude diagrams (Sargent 1977; Getman et al. 2009), and 850 pc based on $V$ versus $V-I$ color-magnitude diagrams (Mayne et al. 2007). The Gaia distance resolves the inconsistency in the distance estimates and places the cluster at the upper end of the range of previous estimates.

\section{Discussion}

After fitting the sizes and densities with the empirical King 1962 model and constraining the kinematics of the two subclusters, we discuss their possible early conditions, evolutionary paths up to their current state, and their potential future fates.

\subsection{The Possible Early Conditions and Evolutionary Paths of the Eastern Subcluster}

There is clear evidence that the east subcluster has undergone gas expulsion due to the radiation and winds of the $\mathrm{O} 7$ star HD 217086. The 2MASS extinction maps show that the gas measured in the east is concentrated around the edge of the subcluster and mostly dispersed in the center of the cluster (Gutermuth et al. 2011a; Allen et al. 2012). With a current number of members and gas mass as observed today, the current SFE is $\sim 73 \%$; the stellar mass dominates the gravitational potential of this subcluster.

There are several reasons the east is in a state of expansion due to the gas dispersal. First, simulations of clusters after gas

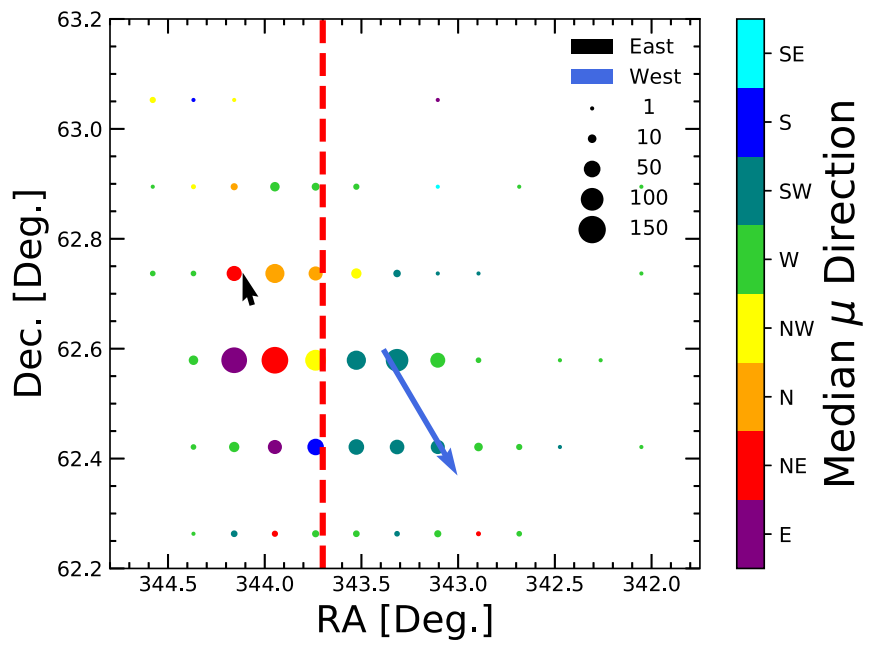

Figure 13. The total proper motions of the east (black arrow) and west (blue arrow) subclusters with the average proper motion of the total cluster removed, demonstrating that the subclusters will not merge but remain separate. The red, vertical dashed line indicates the separation in R.A. of the two subclusters. The colored dots correspond to the median parallax direction at different points in Cep OB3b with the size increasing as the number of stars in the bin agree with the direction of motion. The eastern subcluster has a total proper motion of

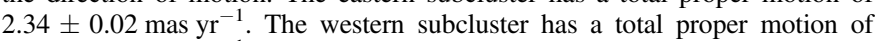
$2.86 \pm 0.03$ mas $\mathrm{yr}^{-1}$. The differential velocity exceeds the escape velocity from the more massive east.

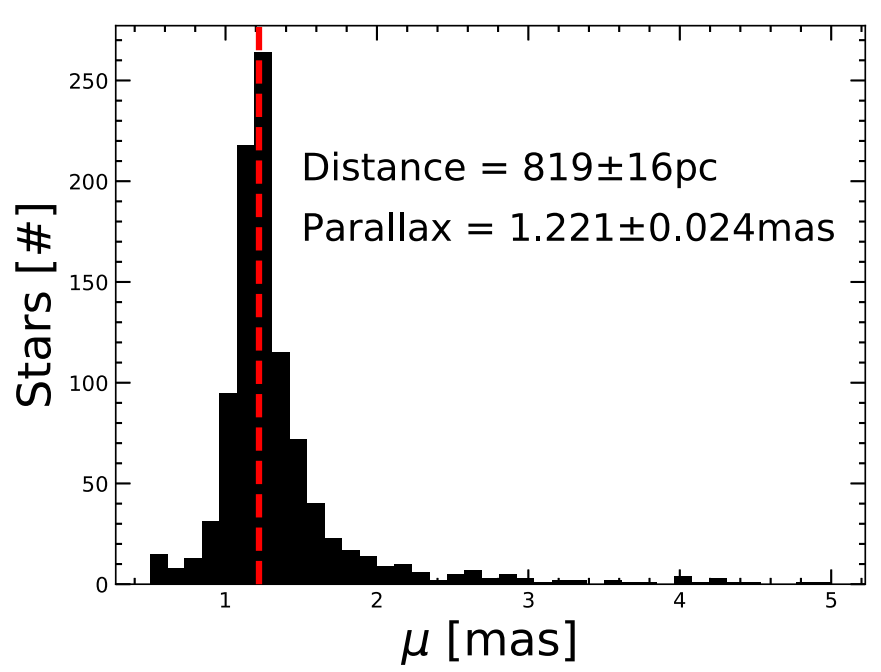

Figure 14. A histogram of objects toward Cep OB3b with parallaxes measured by Gaia in DR2. The median parallax is marked by the dashed, vertical red line, yielding a distance of $819 \pm 16 \mathrm{pc}$. We adopt this distance for Cep OB3b.

dispersal show that clusters expand significantly, with the ratio of the final to initial radii equal to the ratio of the initial to final stellar mass in the case where the cluster remains bound (Baumgardt \& Kroupa 2007; Moeckel \& Bate 2010). An expansion factor of five with an SFE $\sim 0.2$ can lead to a bound cluster if the gas dispersal timescale is slow enough, i.e., several crossing times of the cluster. Second, the circular symmetry of the cluster is unlikely due to relaxation. The crossing time at the current radius is

$$
t_{\text {cross }}=R_{\text {core }} / \sigma_{1 \mathrm{D}}
$$

$0.74 \pm 0.16 \mathrm{Myr}$. This implies $\sim 4$ crossings have occurred since the formation of the cluster assuming an age of 3-4 Myr. 
To determine the relaxation time, we use the two adopted disk fractions and

$$
t_{\text {relax }}=N t_{\text {cross }} / 6 \ln (N / 2),
$$

where $N$ is the number of stars within the eastern subcluster radius. The relaxation time of the east subcluster is $16.6 \pm 3.6 \mathrm{Myr}$ and $14.1 \pm 3.1 \mathrm{Myr}$ using 809 and 664 as $N$, respectively. This indicates that at its current size, the east subcluster does not have time to dynamically relax, and its circular symmetry may instead come from the expansion of the cluster from a more compact, potentially more irregular, structure.

A comparison of the size and density of the eastern subcluster to those of younger clusters shows further evidence for expansion. The east has a core radius of $1.36 \pm 0.30 \mathrm{pc}$, and a peak stellar surface density of 521 and $428 \mathrm{stars} \mathrm{pc}^{-2}$. In comparison, clusters at an early stage in their gas dispersal phase have much smaller sizes and higher densities. We used a combination of Spitzer and Chandra surveys (Megeath et al. 2016) to perform the same King model fits to the ONC and the NGC 2024 cluster in the Orion B cloud, both of which still have significant amounts of molecular gas and contain massive stars. The ONC has a core radius of $0.04462 \pm 0.00003 \mathrm{pc}$ and a central peak stellar density of $1.12 \times 10^{6} \mathrm{stars} \mathrm{pc}^{-2}$. NGC 2024 has a core radius of $0.0305 \pm 0.0038 \mathrm{pc}$ and a central peak stellar density of $5.47 \times 10^{4}$ stars $\mathrm{pc}^{-2}$. These two clusters bracket the number of member stars contained in the east of Cep OB3b: NGC 2024 has $~ 400$ dusty YSOs and the ONC has 3000 dusty YSOs, although the lower stellar density regions of this cluster extend along a $10 \mathrm{pc}$ filament (Megeath et al. 2016).

More recently, Kuhn et al. (2018) found direct evidence in the proper motions from Gaia DR2 that the eastern subcluster, which is referred to as Cepheus B in their paper, is undergoing expansion. They measure parallaxes from 481 members and find a positive radial gradient and significant $(>3 \sigma)$ expansion of Cep B.

Estimating the initial size of the cluster before expansion requires several assumptions. If we assume it began in virial equilibrium $(2 T=U)$, it had $r^{-2}$ density distribution and that the gas and stars have the same spatial distribution, then the outer radius of the cluster is given by $R=\mathrm{GM} / 3 \sigma_{1 \mathrm{D}}^{2}$ (MacLaren et al. 1988), where $M$ is the total mass. The stellar mass, determined by using $0.5 M_{\odot}$ as the average mass, for the 809 and 664 members of the east are $405 M_{\odot}$ and $332 M_{\odot}$, respectively. We adopt a total SFE of 0.2 at the onset of gas dispersal. At that point, $20 \%$ of the mass is in stars and $80 \%$ is in gas. Using SFE $=M_{\text {stars }} / M_{\text {stars }}+M_{\text {gas }}$ and solving for $M_{\text {gas }}$ results in $1620 M_{\odot}$ and $1328 M_{\odot}$, respectively. The total masses are $2025 M_{\odot}$ and $1660 M_{\odot}$, respectively. Assuming a constant velocity dispersion of $2.2 \mathrm{~km} \mathrm{~s}^{-1}$, which is the expectation value for the eastern subcluster for a binary fraction of 0.5 , we obtain an initial outer radius of $0.58 \mathrm{pc}$ and $0.49 \mathrm{pc}$, respectively. The subcluster radius is $2.32 \mathrm{pc}$. If we use $2.2 \mathrm{~km} \mathrm{~s}^{-1}$ as the velocity, then the eastern subcluster has been expanding for $0.76 \mathrm{Myr}$ or $0.80 \mathrm{Myr}$, respectively. Note that the assumptions made are very simplistic. In particular, we would expect the velocity dispersion to decrease with time (Moeckel \& Bate 2010). In this case, the eastern subcluster would have formed in a more compact, dense configuration with a smaller initial radius, which is consistent with observations of younger clusters and from simulations. Furthermore, the initial SFE may range from 0.1 to 0.3 as observed in young, embedded clusters, (Megeath et al. 2016), and the initial conditions may not be virial (e.g., Farias et al. 2018). Note that the value of the SFE can depend on the spatial scale on which it is measured. The inner regions of a protocluster will have a higher SFE value than the edges (see Figure 13 in Parmentier \& Pfalzner 2013).

Although most of the gas has been dispersed, star formation is continuing at the edge of the cluster. Getman et al. (2009) proposed that the $\mathrm{O} 7$ star is creating a radiative-driven implosion (RDI) in the rim of the associated molecular clump, resulting in continued star formation along the edge of the east; see Figure 4 in Allen et al. (2012). Although such an implosion can produce a velocity shift, our results do not detect RV gradient with respect to the location the $\mathrm{O}$ star; however, the gradient could be perpendicular to our line of sight and undetectable from RV motions. Given the small number of protostars in this RDI (Allen et al. 2012), this implosion may only contribute a small fraction of the cluster stars.

It seems clear that the east has expanded since formation; however, it is unclear how many of the stars form a bound cluster. Simulations and theoretical analyses show that clusters where the kinetic energy exceeds the potential energy can still form bound clusters, although at a smaller efficiency. For an assumed SFE of 0.25 and a gas dispersal time of $1 \mathrm{Myr}$, or two crossing times, Baumgardt \& Kroupa (2007) estimated that roughly $55 \%$ of the stars may remain bound. Note that Baumgardt \& Kroupa (2007) assumed the radial density profiles of the stars and of the residual gas in an embedded cluster have the same shape. If the density profile of the stars is steeper than the profile of the gas, which is likely for the inner regions, then the likelihood of the formation of a bound cluster increases substantially (see Adams 2000; Parmentier \& Pfalzner 2013; Shukirgaliyev et al. 2017).

A key parameter is $T /|U|$, known as the virial ratio $(Q)$. Farias et al. (2018), "based on the value of $Q$ just after gas dispersal," estimated the fraction of stars that remains bound after gas dispersal. They compared simple models and simulations to show that the bound number strongly depends on the initial post-gas-dispersal virial ratio. We expect the value of $T /|U|$ to slowly increase as the cluster expands. For a binary fraction of 0.5 , the expectation value of the velocity dispersion for $\log (T /|U|) \sim 0.3$; for this value of $Q, \sim 35 \%$ of the stars will remain bound. At the 25th percentile, the $\log (T /|U|)$ value is 0.1 , which corresponds to $55 \%$ of the members remaining bound and at the 75th percentile, the $\log (T /|U|)$ value is 0.76 , which corresponds to $10 \%$ of the members remaining bound. If the velocity dispersion is closer to those inferred for a binary fraction of 0 , then $10 \%$ or less will remain bound. If the binary fraction is 1 , then up to $95 \%$ of the members remain bound in the 25 th percentile and as few as $20 \%$ members will remain bound in the 75th percentile. Kuhn et al. (2018) found a velocity dispersion of $1.9 \pm 0.2 \mathrm{~km} \mathrm{~s}^{-1}$ and that the cluster is expanding radially. This is similar to the expectation value with a velocity dispersion of $2.2 \mathrm{~km} \mathrm{~s}^{-1}$ for a binary fraction of 0.5 , which is the binary fraction that is most consistent with that of solar-type stars (Duchêne \& Kraus 2013). Although a broad range of outcomes are allowed, we favor where approximately a third of the stars remain bound. 


\subsection{The Possible Early Conditions and Evolutionary Paths of the Western Subcluster}

The smaller western subcluster remains more embedded than the eastern subcluster. We calculate a current SFE between 0.23 and 0.26 depending on the disk fraction, which is much higher than the eastern subcluster. Even though it is similar to SFEs of embedded clusters, the gas is offset to the south of the center of the subcluster, which appears to have at least partially dispersed its gas (Allen et al. 2012). Additional evidence that it is not as dynamically evolved as the eastern subcluster is its elongated morphology and smaller core size $(0.5 \pm 0.3 \mathrm{pc})$.

The lesser degree of dynamical evolution in the west does not necessarily imply a younger age. The west contains one B3 and three B5 stars that do not have the UV radiation of the $\mathrm{O} 7$ star in the eastern subcluster to clear natal gas as quickly. Calculating a crossing time as in Section 5.1 yields $t_{\text {crossing }}=1.45 \pm 0.31 \mathrm{Myr}$. The west has experienced $\sim 3$ crossings since the formation of the cluster, similar to the eastern subcluster, assuming an age of 3-4 Myr. The relaxation time is $\sim 19-22$ Myr for the two disk fractions. This indicates the west is not dynamically relaxed and could maintain its elongated morphology. The core radius is larger than that of the ONC and NGC 2024 (see Section 5.1), and the surface

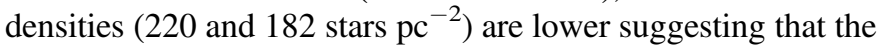
core of the subcluster has expanded.

As the western subcluster evolves, it is unclear how quickly the remaining natal gas will disperse and how the virial ratio will change as a result. It is a reasonable assumption that $T /|U|$ will increase as it expands, but by how much remains unclear. Our range of $\log (T /|U|)$ values (Figure 11) is consistent with a cluster that is currently virialized, although with large error bars.

If this virial ratio is maintained through gas dispersal, then we can again compare our $\log (T /|U|)$ values to Figure 7 in Farias et al. (2018) to determine the fraction of stars that will remain bound as in Section 5.1. For a binary fraction of 0.5, the expectation value of $\log (T /|U|)$ is -0.16 , which yields $\sim 75 \%$ of the stars remaining bound. At the 25 th and the 75 th percentiles, the $\log (T /|U|)$ value is -0.5 and 0.47 , which corresponds to $98 \%$ and $20 \%$ of members remaining bound, respectively. If the velocity dispersion is closer to those inferred for a binary fraction of 0 , then the expectation value of $\log (T /|U|)$ is 0.27 , resulting in $30 \%$ of the stars remaining bound. For the 25 th and 75 th percentiles, as many as $50 \%$ and as few as $10 \%$ of the members will remain bound, respectively. If the binary fraction is 1 , the expectation value results in $95 \%$ of the stars remaining bound. If we take the 25 th and 75 th percentiles, the number of remaining members will be $100 \%$ and $30 \%$, respectively. Although a broad range of outcomes is allowed, we favor where approximately $75 \%$ of the stars remain bound. Baumgardt \& Kroupa (2007) found that a combination of very slow gas expulsion and a very weak external tidal field with an initial SFE of $33 \%$ can produce a bound cluster with $90 \%$ of stars remaining bound. The current SFE is $26 \%$, and it is possible that the west initially had an SFE of $\sim 30 \%$. If the timescale of gas dispersal is prolonged, which is the likely case in the west, then the fraction of bound stars may be higher (Baumgardt \& Kroupa 2007).

\subsection{Fate of Cep $\mathrm{OB} 3 \mathrm{~b}$}

Our values for the virial ratios are consistent with Cep OB3b forming two bound subclusters, each with $\sim 300$ stars. These two subclusters would be found within an expanding association of stars from the lower density halo surrounding both of the subclusters, as well as the members ejected from the subclusters. The associations would have more stars than the clusters combined.

To determine whether the two subclusters may merge into a single cluster, we compare the escape velocity to the relative motions of the two subclusters. The escape velocity is $v_{\mathrm{esc}}=\left(2 G M_{\mathrm{tot}} / R\right)$, where $M_{\mathrm{tot}}$ is the combined stellar mass of the east and west. We use the most optimistic masses of stars, i.e., $(809+501) \times 0.5 M_{\odot}$ and a separation of the subclusters of $5 \mathrm{pc}$ as $R$. This results in an escape velocity of $1.06 \mathrm{~km} \mathrm{~s}^{-1}$. The total proper motion of Cep OB3b is subtracted off the proper motions of the subclusters. The east is moving at $-0.47 \mathrm{~km} \mathrm{~s}^{-1}$, and the west is moving at $1.55 \mathrm{~km} \mathrm{~s}^{-1}$ for a difference in $2.02 \mathrm{~km} \mathrm{~s}^{-1}$. Considering that the masses of the subclusters will be lower after gas dispersal and that the actual separation is likely to be larger than the projected separation, this escape velocity should be considered as an upper limit. Thus, the two subclusters are moving away from each other at a velocity that exceeds the escape velocity and will only increase in separation, forming a double cluster.

This result seems exceptional in light of the fact that $\sim 7 \%$ of embedded clusters form open clusters (Lada \& Lada 2003). This might be due to unusual conditions in the Cep OB3b cluster. Alternatively, there might be other factors that contribute to the dissipation of clusters (see Moeckel et al. 2012), particularly when a realistic range of masses is used. Stellar dynamical effects come into play when binaries and a realistic range of masses are used (Moeckel et al. 2012). Alternatively, the tidal forces between the clusters, and between the clusters and the molecular clouds, may disrupt the clusters or strip members. It should also be noted that the large uncertainties in the velocity dispersions translate into large uncertainties in the dynamical outcomes. If both subclusters form bound clusters, then the study of Cep OB3b will help establish the cluster properties and environmental factors that produce bound clusters.

\subsection{The Effect of Binarity on the Velocity Dispersions of Young Clusters}

One of the results of our analysis is that the adopted binary fraction strongly influences the PDFs for the cluster velocity dispersions, as demonstrated in Figure 8. This importance can be further demonstrated by a simple comparison of the expected velocity dispersion due to binarity compared to that from clusters. We compare these by approximating the ratio of the velocity dispersion from cluster motions to that from binary motions.

The $1 \mathrm{D}$ velocity dispersion of a barely bound $(U=T)$ cluster is given by

$$
\sigma_{1 \mathrm{D}}^{2}=\alpha \frac{G M}{R}
$$

where $M$ is the total mass of the cluster, $R$ is the core radius of the cluster, and $\alpha$ depends on the radial density distribution of the spherically symmetric cluster. We adopt the value of $\alpha=2 / 5$, which is that for a uniform density cluster. For a 
cluster, the observed velocity dispersion due to the motions of binaries is approximately

$$
\sigma_{\text {bin }}^{2}=f_{\text {bin }} \beta \frac{G m}{r},
$$

where $m$ is the combined mass of the system, $r$ is the typical semimajor axis, $f_{\text {bin }}$ is the binary fraction, and $\beta$ takes into account the effect of orbital inclinations and orbital phases on the line-of-sight velocity. The 0.5 term assumes that the reduced mass is half the combined mass. We adopt $\beta=2 / 3$, $m=1.5 M_{\odot}$, and a semimajor axis of $45 \mathrm{au}$; this is the peak of the log-normal semimajor axis distribution for solar-mass field stars (Duchêne \& Kraus 2013). Throughout the following analysis, we assume $f_{\text {bin }}=0.5$. The adopted system mass of $1.5 M_{\odot}$ assumes that the stars with measured velocity dispersions have masses of $0.75 M_{\odot}$ each.

To compare the magnitudes of the binary motions and motions within a cluster, we consider the ratio of the two velocities dispersions, which is given by

$$
\frac{\sigma_{\text {bin }}^{2}}{\sigma_{1 \mathrm{D}}^{2}}=f_{\text {bin }}\left(\frac{\beta}{\alpha}\right)\left(\frac{m_{\text {bin }}}{N_{\star} m_{\star}}\right)\left(\frac{R}{r}\right),
$$

where $N_{\star}$ is the total number of stellar systems (including single and multiple stars) in the cluster and $m_{\star}$ is the average mass of the cluster members. The adopted mass of the cluster members is $m_{\star}=0.75\left(1+f_{\text {bin }}\right) M_{\odot}$, where the average mass of an individual star with a measured velocity is assumed to be $0.75 M_{\odot}$. For a cluster with $N_{\star}=500$, we find that for the ratio of velocity dispersions to be equal to or less than 1 (i.e., that the cluster motions are equal to or exceed those of the binaries), the cluster radius must be $\leqslant 0.1 \mathrm{pc}$. This ratio increases linearly with the number of stars. Thus, for all but the youngest, most compact clusters, the motions of the binaries dominate the observed velocity dispersion.

For embedded clusters, the dominant mass is that of the molecular gas. This requires us to include the SFE in Equation (6); the SFE is the ratio of the mass in stars over the combined stellar and gas mass. Specifically, the value of $N_{\star} m_{\star}$ must be replaced with $N_{\star} m_{\star} / \mathrm{SFE}$. If we adopt $\mathrm{SFE}=0.2$, a common value for young clusters at earlier phases of gas dispersal (e.g., Megeath et al. 2016), then the velocity dispersion ratio is unity or less for $R \leqslant 0.5 \mathrm{pc}$. Thus, in the case of young embedded clusters, which have typical radii of $0.5 \mathrm{pc}$, the contribution of cluster motions can exceed or equal those from binaries due to the effect of the gas mass on the cluster motions. As the gas is dispersed and the cluster expands, binaries will begin to dominate the observed distribution if it remains in virial equilibrium while expanding.

\section{Summary}

We present a study of the spatial structure and kinematics of Cep OB3b, a young cluster currently in a late stage of gas dispersal. Cep OB $3 b$ is one of the closest examples of a $\sim 3-5 \mathrm{Myr}$, large ( 3000 total members) cluster. The cluster is broken into two subclusters: the east, which contains an $\mathrm{O} 7$ star, and west, which has several B stars. Using the combined sample of members identified with IR excesses or X-ray detections (Allen et al. 2012), the structure, density structures, and deviation from circular symmetry of the two subclusters are determined from fitting the empirical density law of King (1962). We present new RV measurements from Hectoschelle of 499 stars; 109 of these are likely confirmed members of Cep OB3b with youth indicators. After excluding potential binaries and applying an $R$ cut, $3 \sigma$ clipping, and subcluster radius cut to the data, we are left with 52 stars in our RV analysis.

1. We derive the distance to Cep OB3b using known members found in the Gaia DR2 catalog. The distance is $819 \pm 16 \mathrm{pc}$.

2. Fits to the empirical King (1962) models yield the properties of the cluster. For the eastern subcluster, we find a core radius of $1.36 \pm 0.30 \mathrm{pc}$ and a peak density of 428-521 stars $\mathrm{pc}^{-2}$, depending on the adopted disk fraction. For the western subcluster, we find a core radius of $0.52 \pm 0.11 \mathrm{pc}$ and a peak density of 284 and 342 stars $\mathrm{pc}^{-2}$. The subcluster radii of the subclusters are $2.32 \pm 0.19 \mathrm{pc}$ in the east, with 664-809 stars within this border, and $3.1 \pm 1.0 \mathrm{pc}$ in the west, with 332-402 stars within this border.

3. We have determined the posterior probability distribution for the velocity dispersion of each subcluster. This analysis includes a contribution due to binaries for an assumed binary fraction. The peaks of the distributions in the east are $2.8_{-0.7}^{+0.6} \mathrm{~km} \mathrm{~s}^{-1}, 2.2 \pm 1.0 \mathrm{~km} \mathrm{~s}^{-1}$, and $1.15_{-0.95}^{+0.80} \mathrm{~km} \mathrm{~s}^{-1}$ for a binary fractions of $0,0.5$, and 1 , respectively, The expectation values are $2.8_{-0.7}^{+0.6} \mathrm{~km} \mathrm{~s}^{-1}$, $1.9 \pm 1.0 \mathrm{~km} \mathrm{~s}^{-1}$, and $1.4_{-1.0}^{+0.8} \mathrm{~km} \mathrm{~s}^{-1}$ for binary fractions of $0,0.5$, and 1 , respectively. The west has velocity dispersion peaks at $1.5_{-0.50}^{+0.70} \mathrm{~km} \mathrm{~s}^{-1}, 0.5_{-0.5}^{+0.8} \mathrm{~km} \mathrm{~s}^{-1}$, and $0.3_{-0.3}^{+0.6} \mathrm{~km} \mathrm{~s}^{-1}$ with expectation values of $1.5_{-0.50}^{+0.70} \mathrm{~km} \mathrm{~s}^{-1}$, $1.1_{-0.5}^{+0.8} \mathrm{~km} \mathrm{~s}^{-1}$, and $1.0_{-0.3}^{+0.6} \mathrm{~km} \mathrm{~s}^{-1}$ for binary fractions of $0,0.5$, and 1, respectively. Using Gaia DR2 proper motions, Kuhn et al. (2018) found a velocity dispersion of $1.9 \pm 0.2 \mathrm{~km} \mathrm{~s}^{-1}$ for the east.

4. A comparison of the eastern subcluster with the NGC 2024 and ONC cluster indicates that this cluster has a much larger core radius and lower central density. It also is circularly symmetric. This is evidence that this cluster has undergone significant expansion. Although the western subcluster is more compact than the eastern one and shows a significant asymmetry, it still has a larger core radius and lower central density than the two Orion clusters. This subcluster also appears to have undergone some degree of expansion.

5. The inferred ratio of the kinetic to potential energy of the eastern subcluster, $T /|U|$, shows that this ratio depends strongly on the adopted binary fraction for the stars. For a binary fraction of 0 and $0.5, \log (T /|U|)>0$, suggesting the subcluster will undergo expansion. For a binary fraction of 1, which is unlikely, there is a moderate probability that the cluster could be bound. We conclude that this subcluster most likely has a ratio greater than 1 and is expanding.

6. A similar analysis for the western subcluster shows $\log (T /|U|) \sim 0$ for binary fractions of 0.5 and 1 , but $\log$ $(T /|U|)>0$ for a binary fraction of 0 . We conclude that this subcluster is close to bound and may be virialized.

7. Accounting for unresolved binaries is important to accurately probe the dynamical properties of young clusters, particularly after gas dispersal. The binary motions can dominate the motions of the cluster, and 
this is particularly important when limited to solar-mass stars with a high fraction of multiplicity.

8. For the binary fraction of 0.5 , we find that the eastern subcluster is more likely in a state of expansion. Comparisons to nbody simulations suggest that $35 \%$ of the member stars may remain to form a bound cluster. In contrast, the western subcluster may be near virial equilibrium where close to $75 \%$ of the members remain bound. These two different outcomes may be driven by the rapid dispersal of gas in the eastern subcluster, due to the presence of an $\mathrm{O} 7$ star. In contrast, the western subcluster, which still contains a significant mass of gas, only contains massive stars of spectral type B3 or later.

9. A likely outcome is that the two subclusters will form bound clusters with 300 stars. An analysis of the bulk proper motion of the two subclusters using Gaia DR2 shows that the subclusters are moving away from each other with $\sim 2 \mathrm{~km} \mathrm{~s}^{-1}$, and they are not bound. Thus, Cep OB3b may be forming two, independent bound clusters. Given that only $\sim 7 \%$ of embedded clusters survive to form bound clusters, this is a very rare outcome, suggesting that the physical conditions in Cep OB3b are highly conducive to bound cluster formation. Alternatively, other factors that have been ignored in our analysis, such as internal dynamics of clusters or tidal forces, may play an important role in the disruption of clusters; if this is the case, the nascent bound clusters in Cep OB3b may still be disrupted.

We wish to thank the anonymous referee for excellent suggestions that improved the quality of this manuscript. The authors are also grateful to $\mathrm{M}$. Kounkel for useful discussions and comments throughout the progress of this project. This work was supported by NSF grant AST-1009564 to S.T.M. R. A.G. gratefully acknowledges funding support from NASA ADAP grants NNX11AD14G, NNX15AF05G, and NNX17AF24G; NASA JPL/Caltech contract 1489384; and NSF grant AST 1636621 in support of TolTEC, the next generation mm-wave camera for LMT. Data presented herein were obtained at the MMT Observatory at Fred Lawrence Whipple Observatory on Mount Hopkins, AZ, USA. This work made use of the SIMBAD database, the Vizier database, the NASA Astrophysics Data System, and the data products from the Two Micron All Sky Survey, which is a joint project of the University of Massachusetts and the Infrared Processing and Analysis Center/California Institute of Technology, funded by the National Aeronautics and Space Administration and the NSF.

This work has made use of data from the European Space Agency (ESA) mission Gaia (https://www.cosmos.esa.int/ gaia), processed by the Gaia Data Processing and Analysis Consortium (DPAC, https://www.cosmos.esa.int/web/gaia/ dpac/consortium). Funding for the DPAC has been provided by national institutions, in particular the institutions participating in the Gaia Multilateral Agreement.

\section{Appendix Calcium II H \& K Emission}

$\mathrm{Ca}$ II $\mathrm{H}$ and $\mathrm{K}$ line emission is an indicator of youth and a way of identifying diskless pre-MS stars that do not have detectable X-ray emission. Emission in the H\&K lines (3968.5 $\AA$ and $3933.7 \AA$, respectively) in a spectrum may

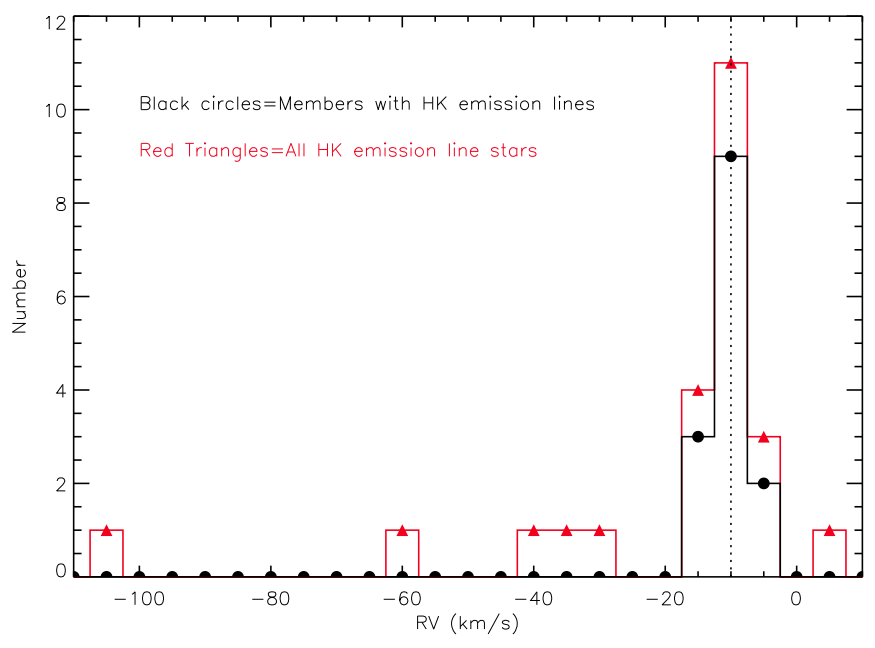

Figure 15. Histogram of RVs of stars marked with HK emission lines and $R$ values larger than 5.0. The histograms marked by the black circles are for objects with youth indicators, and the red triangles are for all objects with H\&K emission lines. There is a clear peak of the objects with youth indicators at $-10 \mathrm{~km} \mathrm{~s}^{-1}$ (dotted line) that corresponds to the $V_{\text {lsr }}$ peak of Cep OB3b.

indicate magnetic activity in the chromosphere. As stars contract onto the main sequence, their magnetic fields can be stronger than when they reach the main sequence. The increased magnetic field may be due to rapid rotation of stars in the pre-MS phase (Johns-Krull et al. 1999). We used the Hectospec data to determine if any of our objects had H\&K emission. When we observed emission in the line core, the object was marked to have H\&K emission. Fifteen objects with Ca II $\mathrm{H} \& \mathrm{~K}$ line emission in our sample had no previous indications of youth as well as 25 objects that had at least one youth indicator already.

As seen in Figure 15, most (83\%) objects with H\&K emission have RVs within $3 \sigma$ of the average RV of the cluster, whereas $46 \%$ of the objects without youth indicators are within $3 \sigma$ of the average RV of the cluster. The outliers with H\&K emission may be binaries that we simply have not detected or may indicate that $\sim 20 \%$ of the objects identified by H\&K emission may be contaminants.

\section{ORCID iDs}

J. J. Prchlik (1) https://orcid.org/0000-0002-3517-9421

R. A. Gutermuth (1) https://orcid.org/0000-0002-6447-899X

S. T. Megeath (1) https://orcid.org/0000-0001-7629-3573

J. L. Pipher (i) https://orcid.org/0000-0002-0628-9605

S. Wolk (i) https://orcid.org/0000-0002-0826-9261

\section{References}

Adams, F. C. 2000, ApJ, 542, 964

Adams, J. D., Stauffer, J. R., Monet, D. G., Skrutskie, M. F., \& Beichman, C. A. 2001, AJ, 121, 2053

Allen, L., Megeath, S. T., Gutermuth, R., et al. 2007, in Protostars and Planets V (Tuscon: University of Arizona Press), 361

Allen, T. S., Gutermuth, R. A., Kryukova, E., et al. 2012, ApJ, 750, 125

Allen, T. S., Prchlik, J. J., Megeath, S. T., et al. 2014, ApJ, 786, 113

Baumgardt, H., \& Kroupa, P. 2007, MNRAS, 380, 1589

Blaauw, A., Hiltner, W. A., \& Johnson, H. L. 1959, ApJ, 130, 69

Boily, C. M., \& Kroupa, P. 2003, MNRAS, 338, 673

Carpenter, J. M. 2000, AJ, 120, 3139

Chen, H.-C., \& Ko, C.-M. 2009, ApJ, 698, 1659

Coelho, P., Barbuy, B., Meléndez, J., Schiavon, R. P., \& Castilho, B. V. 2005, A\&A, 443, 735 
Cottaar, M., Covey, K. R., Foster, J. B., et al. 2015, ApJ, 807, 27 Cottaar, M., Meyer, M. R., \& Parker, R. J. 2012, A\&A, 547, A35 Dahm, S. E., \& Simon, T. 2005, AJ, 129, 829

Da Rio, N., Tan, J. C., Covey, K. R., et al. 2017, ApJ, 845, 105

Da Rio, N., Tan, J. C., \& Jaehnig, K. 2014, ApJ, 795, 55

Duchêne, G., \& Kraus, A. 2013, ARA\&A, 51, 269

Evans, N. J., II, Dunham, M. M., Jørgensen, J. K., et al. 2009, ApJS, 181, 321

Farias, J. P., Fellhauer, M., Smith, R., Domínguez, R., \& Dabringhausen, J. 2018, MNRAS, 476, 5341

Farias, J. P., Smith, R., Fellhauer, M., et al. 2015, MNRAS, 450, 2451

Foster, J. B., Cottaar, M., Covey, K. R., et al. 2015, ApJ, 799, 136

Fûrész, G., Hartmann, L. W., Megeath, S. T., Szentgyorgyi, A. H., \& Hamden, E. T. 2008, ApJ, 676, 1109

Fürész, G., Hartmann, L. W., Szentgyorgyi, A. H., et al. 2006, ApJ, 648, 1090 Getman, K. V., Feigelson, E. D., Luhman, K. L., et al. 2009, ApJ, 699, 1454 Geyer, M. P., \& Burkert, A. 2001, MNRAS, 323, 988

Goodwin, S. P. 2009, Ap\&SS, 324, 259

Gutermuth, R. A., Allen, T., Megeath, T., Pipher, J., \& Wolk, S. 2011a, AAS Meeting Abstracts, 43, 258.19

Gutermuth, R. A., Megeath, S. T., Myers, P. C., et al. 2009, ApJS, 184, 18

Gutermuth, R. A., Megeath, S. T., Pipher, J. L., et al. 2005, ApJ, 632, 397

Gutermuth, R. A., Pipher, J. L., Megeath, S. T., et al. 2011b, ApJ, 739, 84

Heyer, M. H., Carpenter, J. M., \& Ladd, E. F. 1996, ApJ, 463, 630

Hillenbrand, L. A., \& Hartmann, L. W. 1998, ApJ, 492, 540

Jeffries, R. D., Jackson, R. J., Cottaar, M., et al. 2014, A\&A, 563, A94

Jeffries, R. D., Maxted, P. F. L., Oliveira, J. M., \& Naylor, T. 2006, MNRAS, 371, L6

Johns-Krull, C. M., Valenti, J. A., Hatzes, A. P., \& Kanaan, A. 1999, ApJL, 510, L41

King, I. 1962, AJ, 67, 471

Kounkel, M., Hartmann, L., Mateo, M., \& Bailey, J. I., III 2017, ApJ, 844, 138

Kounkel, M., Hartmann, L., Tobin, J. J., et al. 2016, ApJ, 821, 8

Kuhn, M. A., Feigelson, E. D., Getman, K. V., et al. 2014, ApJ, 787, 107

Kuhn, M. A., Hillenbrand, L. A., Sills, A., Feigelson, E. D., \& Getman, K. V. 2018, arXiv:1807.02115

Kurtz, M. J., \& Mink, D. J. 1998, PASP, 110, 934

Kurucz, R. L. 1993, PhST, 47, 110
Lada, C. J., \& Lada, E. A. 2003, ARA\&A, 41, 57

Lada, C. J., Margulis, M., \& Dearborn, D. 1984, ApJ, 285, 141

Lindegren, L., Hernández, J., Bombrun, A., et al. 2018, A\&A, 616, A2

Littlefair, S. P., Naylor, T., Mayne, N. J., Saunders, E. S., \& Jeffries, R. D. 2010, MNRAS, 403, 545

MacLaren, I., Richardson, K. M., \& Wolfendale, A. W. 1988, ApJ, 333, 821

Markwardt, C. B. 2009, in ASP Conf. Ser. 411, Astronomical Data Analysis Software and Systems XVIII, ed. D. A. Bohlender, D. Durand, \& P. Dowler (San Francisco, CA: ASP), 251

Marrese, P. M., Marinoni, S., Fabrizio, M., \& Altavilla, G. 2018, arXiv:1808.09151

Mayne, N. J., Naylor, T., Littlefair, S. P., Saunders, E. S., \& Jeffries, R. D. 2007, MNRAS, 375, 1220

Megeath, S. T., Gutermuth, R., Muzerolle, J., et al. 2016, AJ, 151, 5

Moeckel, N., \& Bate, M. R. 2010, MNRAS, 404, 721

Moeckel, N., Holland, C., Clarke, C. J., \& Bonnell, I. A. 2012, MNRAS, 425,450

Moscadelli, L., Reid, M. J., Menten, K. M., et al. 2009, ApJ, 693, 406

Munari, U., Sordo, R., Castelli, F., \& Zwitter, T. 2005, A\&A, 442, 1127

Ortiz-León, G. N., Loinard, L., \& Dzib, S. A. 2018, arXiv:1812.02360

Parmentier, G., \& Pfalzner, S. 2013, A\&A, 549, A132

Pelupessy, F. I., \& Portegies Zwart, S. 2012, MNRAS, 420, 1503

Proszkow, E.-M., Adams, F. C., Hartmann, L. W., \& Tobin, J. J. 2009, ApJ, 697, 1020

Rapson, V. A., Pipher, J. L., Gutermuth, R., et al. 2014, ApJ, 794, 124

Rigliaco, E., Wilking, B., Meyer, M. R., et al. 2016, A\&A, 588, A123

Sacco, G. G., Spina, L., Randich, S., et al. 2017, A\&A, 601, A97

Sargent, A. I. 1977, ApJ, 218, 736

Shukirgaliyev, B., Parmentier, G., Berczik, P., \& Just, A. 2017, A\&A, 605, A119

Sicilia-Aguilar, A., Hartmann, L. W., Szentgyorgyi, A. H., et al. 2005, AJ, 129,363

Szentgyorgyi, A. 2006, NewAR, 50, 326

Szentgyorgyi, A. H., Cheimets, P., Eng, R., et al. 1998, Proc. SPIE, 3355, 242

Tobin, J. J., Hartmann, L., Fúrész, G., Hsu, W.-H., \& Mateo, M. 2015, AJ, 149, 119

Tobin, J. J., Hartmann, L., Furesz, G., Mateo, M., \& Megeath, S. T. 2009, ApJ, 697, 1103

Tonry, J., \& Davis, M. 1979, AJ, 84, 1511 